\title{
Deep Features based Hierarchical Classification Scheme for Face Recognition in Heterogeneous Environments
}

\author{
Neeru Narang
}

Follow this and additional works at: https://researchrepository.wvu.edu/etd

\section{Recommended Citation}

Narang, Neeru, "Deep Features based Hierarchical Classification Scheme for Face Recognition in Heterogeneous Environments" (2017). Graduate Theses, Dissertations, and Problem Reports. 6294. https://researchrepository.wvu.edu/etd/6294

This Dissertation is protected by copyright and/or related rights. It has been brought to you by the The Research Repository @ WVU with permission from the rights-holder(s). You are free to use this Dissertation in any way that is permitted by the copyright and related rights legislation that applies to your use. For other uses you must obtain permission from the rights-holder(s) directly, unless additional rights are indicated by a Creative Commons license in the record and/ or on the work itself. This Dissertation has been accepted for inclusion in WVU Graduate Theses, Dissertations, and Problem Reports collection by an authorized administrator of The Research Repository @ WVU.

For more information, please contact researchrepository@mail.wvu.edu. 


\section{ERBIUM ALLOYED AIN THIN FILMS: STRUCTURAL, PIEZOELECTRIC AND \\ MAGNETIC PROPERTIES}

By

Vishal Narang

Dissertation Submitted to the Eberly College of Arts and Sciences at West Virginia University in partial fulfillment of the requirements for the degree of

Doctor of Philosophy

In

Physics

Approved by

Mohindar S. Seehra, Ph.D., Committee Chairperson

Dimitris Korakakis, Ph.D. Committee Co-Chairperson

Wathiq Abdul-Razzaq, Ph.D.

Alan D. Bristow, Ph.D.

Cheng Cen, Ph.D.

Department of Physics

Morgantown, West Virginia

2015

Keywords: Piezoelectrics, AlN, AlN:Er, $\mathrm{Er}_{2} \mathrm{O}_{3}$, Magnetism, Exchange Interaction Copyright 2015 [Vishal Narang] 


\title{
ABSTRACT \\ Erbium alloyed AIN thin films: Structural, piezoelectric and magnetic properties
}

\author{
Vishal Narang
}

This research was undertaken to determine the effect of Erbium in Erbium-alloyed AlN thin films on their structural, piezoelectric and magnetic properties. For this purpose, Erbium-alloyed AlN thin films with Er concentration of 0, 1, 3 and 4 atomic percent were deposited on (001) p-type Si substrates by reactive magnetron sputtering. The samples were characterized by $\mathrm{x}$-ray diffraction (XRD), x-ray photoelectron spectroscopy (XPS) and Variable Angle Spectroscopic Ellipsometry (VASE). XRD measurements showed that Er alloying leads to preferential c-axis film deposition with a decrease in grain size and an increase in the lattice constant. XPS analysis was used to determine the Er concentration and its possible chemical state. VASE measurements were used to determine the thickness and refractive indices of the thin films. Refractive indices were used as metric to determine the structural order of polycrystalline thin films. Piezoelectric measurements showed that Er alloying results in the higher magnitude of the piezoelectric coefficient $\mathrm{d}_{33}$. Values of as high as $15.0 \mathrm{pm} / \mathrm{V}$ is measured for AlN:Er whereas the maximum value obtained for pure AlN thin films is $6.9 \mathrm{pm} / \mathrm{V}$.

In order to determine the electronic state of Er in AlN:Er thin films, magnetization (M) of the samples was measured as a function of temperature $(2 \mathrm{~K}$ to $300 \mathrm{~K})$ and magnetic field $(\mathrm{H})$ up to $90 \mathrm{kOe}$ at select temperatures. In particular, the focus was to determine whether Er in AlN:Er is present as Er metal, $\mathrm{Er}_{2} \mathrm{O}_{3}$ or $\mathrm{Er}^{3+}$ substituting for $\mathrm{Al}^{3+}$. For this purpose, a detailed investigation of the magnetic properties of Er metal sample and a powder sample of $\mathrm{Er}_{2} \mathrm{O}_{3}$ was also carried out for comparison purposes. Analysis of the results from these investigations showed that $\mathrm{Er}$ in $\mathrm{AlN}$ : $\mathrm{Er}$ is present as $\mathrm{Er}^{3+}$ substituting for $\mathrm{Al}^{3+}$ in concentrations which are in good agreement with those determined from XPS. Lattice expansion of AlN:Er compared to AlN observed in XRD measurements is hence attributed to larger size $\mathrm{Er}^{3+}$ replacing $\mathrm{Al}^{3+}$.

New results obtained from the analysis of $\mathrm{M}$ vs. $\mathrm{H}$ data in $\mathrm{Er}_{2} \mathrm{O}_{3}$ showed the presence of two magnetic-field induced transitions below its Nèel temperature $\mathrm{T}_{\mathrm{N}} \simeq 3.3 \mathrm{~K}$. Measurements of magnetization $(\mathrm{M})$ vs. magnetic field $(\mathrm{H})$ at $2 \mathrm{~K}$ show a transition at $\mathrm{H}_{\mathrm{SF}} \simeq 15 \mathrm{kOe}$ which is interpreted in terms of spin flop transition and a second observed transition at $\mathrm{H}_{\mathrm{sp}} \simeq 31 \mathrm{kOe}$ represents field-aligned ferromagnetism in $\mathrm{Er}_{2} \mathrm{O}_{3}$. This interpretation is confirmed by theoretical analysis. Magnetic field dependence of the Neel temperature $T_{N}$ is determined and it is observed to follow the equation $T_{N}(H)=$ $\mathrm{T}_{\mathrm{N}}(0)-\mathrm{D}_{1} \mathrm{H}^{2}$ expected for antiferromagnets. From the temperature dependence of magnetic susceptibility of $\mathrm{Er}_{2} \mathrm{O}_{3}$ above $\mathrm{T}_{\mathrm{N}}$ and its fit to the Curie-Weiss law, the molecular field model is used to determine the nearest neighbor (nn) and next nearest neighbor ( $\mathrm{nnn}$ ) exchange constants $\mathrm{J}_{1}=-0.57 \mathrm{~K}$ and $\mathrm{J}_{2}=-3.38 \mathrm{~K}$ respectively. The determined values of $\mathrm{J}_{1}$ and $\mathrm{J}_{2}$ are then used to calculate the magnetic field strength for field-induced ferromagnetic transition and it agrees very well with experimental value of $31 \mathrm{kOe}$.

The major conclusions from this research are that Erbium in Erbium alloyed AlN thin films is present as $\mathrm{Er}^{3+}$ substituting for $\mathrm{Al}^{3+}$ thereby increasing its lattice constant, preferentially ordering the thin films and significantly enhancing the piezoelectric coefficient. For the related compound of $\operatorname{Er}_{2} \mathrm{O}_{3}$, two new magnetic-field induced transitions and associated magnitudes of exchange parameters are reported. 


\section{ACKNOWLEDGEMENTS}

I would like to use this opportunity to thank people who helped me in completing the research presented in this dissertation.

First and foremost of all, I would like to express my deep heartfelt thanks to my advisor and committee chair Dr. Mohindar S. Seehra, for his invaluable guidance, patience and encouragement. His hard working nature is always a source of inspiration for me. His ideas guided me in formulating and completing this research. I owe my deepest gratitude to my advisor and committee co-chair, Dr. Dimitris Korakakis, for his support, guidance and technical inputs during my research. Working with Dr. Korakakis enabled me develop a wide range of engineering skills for which I am very thankful to him.

I would like to thank my committee members Dr. Wathiq Abdul-Razzaq, Dr. Alan D. Bristow and Dr. Cheng Cen for their time and efforts put towards this work. Additionally I would like to thank Dr. Kolin Brown, Dr. Wei Ding and Harley Hart for their help in using Shared Research Facilities throughout this work.

I would also like to thank my lab colleagues, both past and present; Dr. Sridhar Kuchibhatla, Dr. Ronak Rahimi, Dr. Lee Rodak, Dr. Kyoungnae Lee, Kelly Pisane, Anand Kadiyala, Vamsi Kumbham, Kenneth Hite and Joshua Justice for their help at various stages of this work.

I owe my thanks to my parents, brother and sisters for their support and trust in me. Last but not least I wish to thank my wife Neeru who shared with me all my successes and failures during this work, and is always a source of inspiration for me. 


\section{TABLE OF CONTENTS}

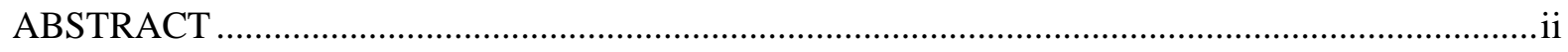

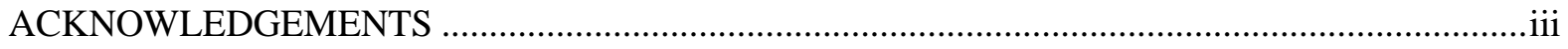

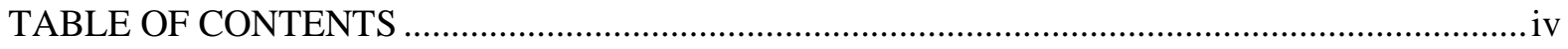

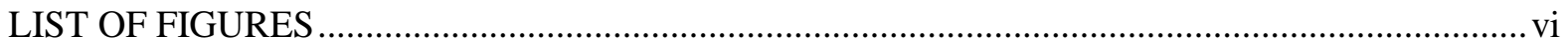

CHAPTER 1 - Introduction and Background........................................................................... 1

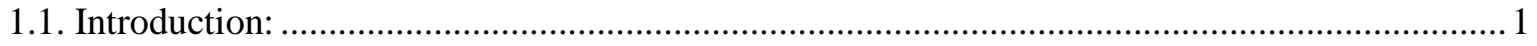

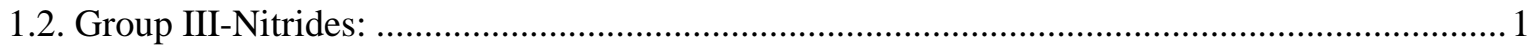

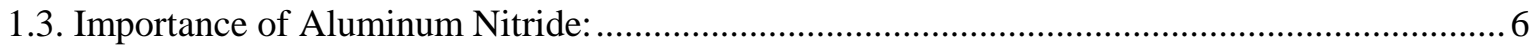

1.4. Rare-earth Doped Materials and AlN:Er: .................................................................... 8

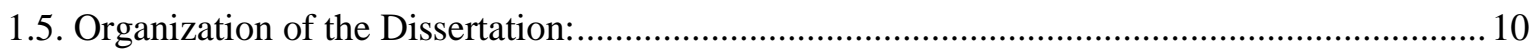

CHAPTER 2 - Sample Preparation and Experimental Procedures .................................................. 12

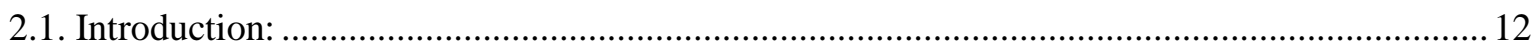

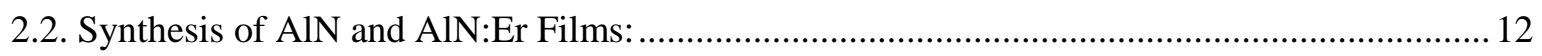

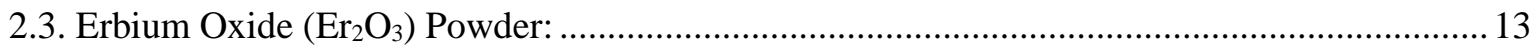

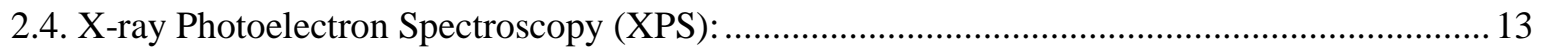

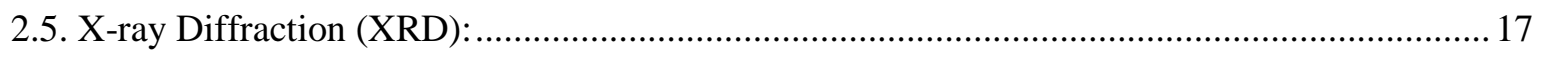

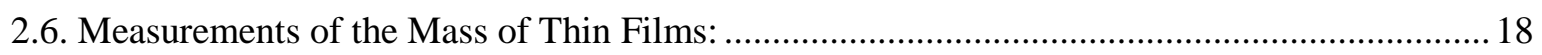

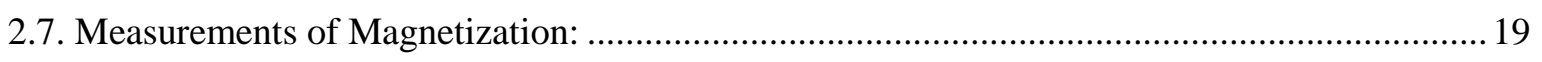

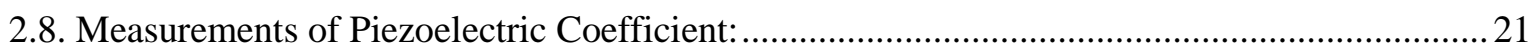

2.9. Platinum Contact Deposition on Thin Films for Piezoelectric Coefficient Measurements:...... 22

CHAPTER 3 - Piezoelectric Properties of AlN:Er Thin Films ........................................................ 25

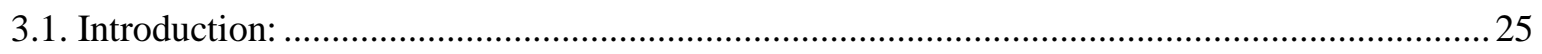

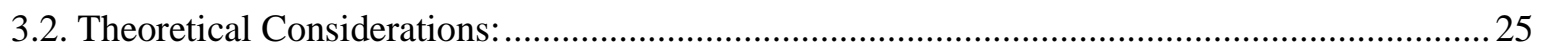

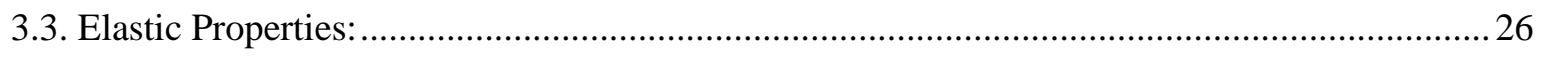

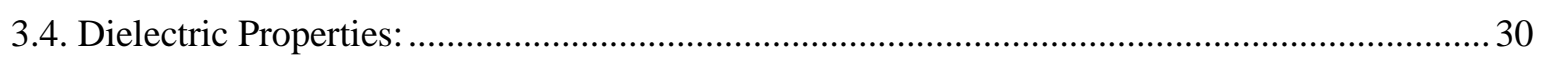

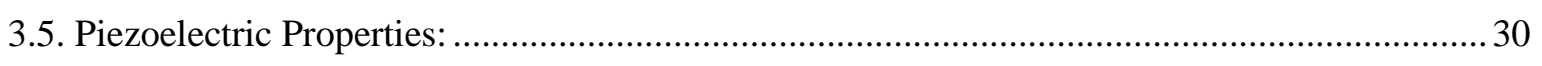

3.6. Experimental Results on Piezoelectric Coefficient of AlN:Er Thin Films: ............................. 31

3.7. Effect of Annealing on the X-ray Diffraction Patterns of AlN:Er Thin Films: ........................ 38 
CHAPTER 4 - Magnetic Characterization of the Electronic State of Er in AlN:Er Films. 39

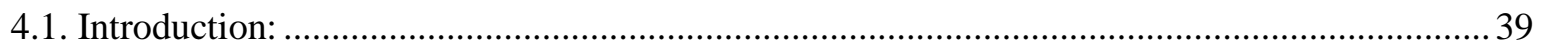

4.2. Curie Law, Curie-Weiss Law and Exchange Interaction: .................................................. 39

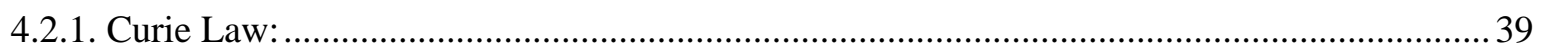

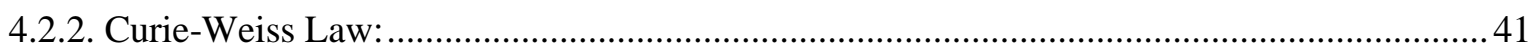

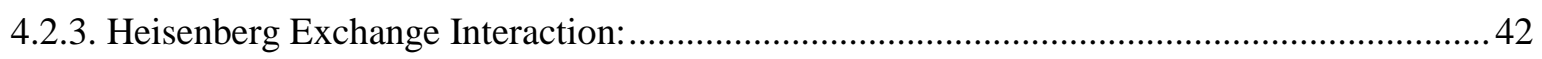

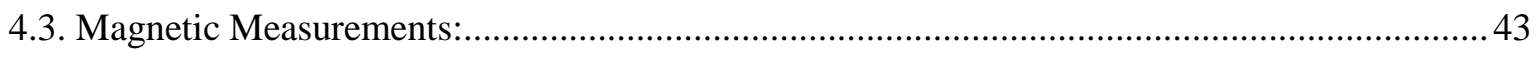

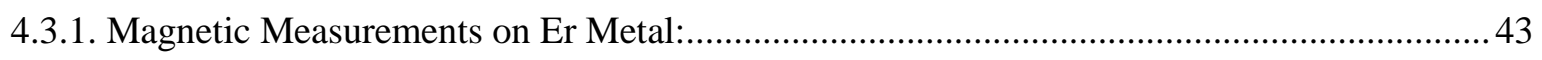

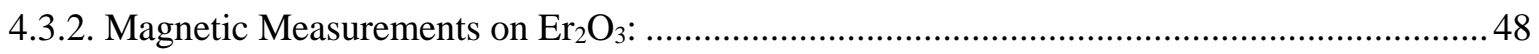

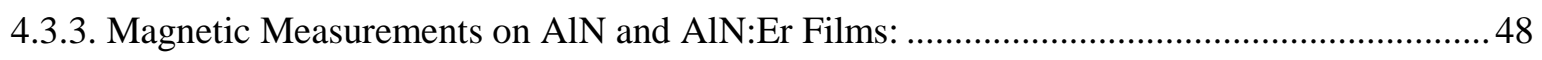

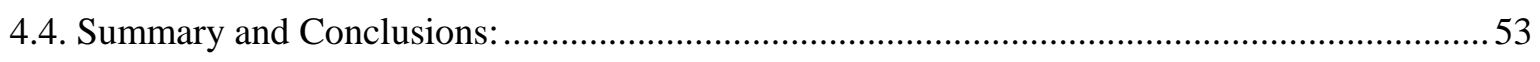

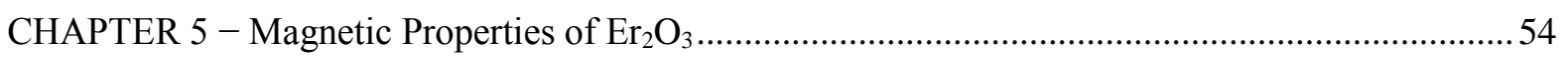

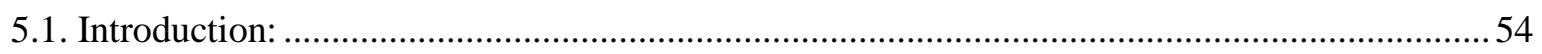

5.2. Previous Studies on the Magnetic Properties of $\mathrm{Er}_{2} \mathrm{O}_{3}$ :....................................................54

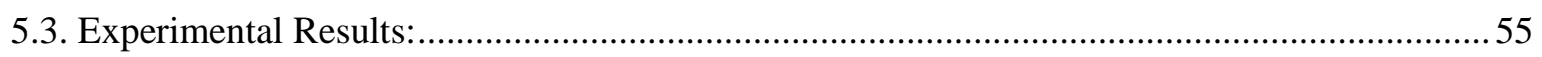

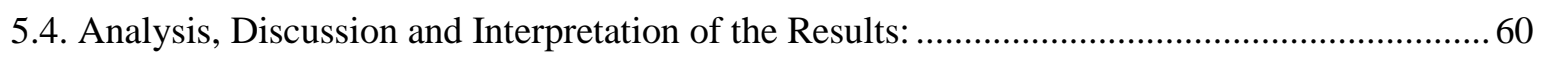

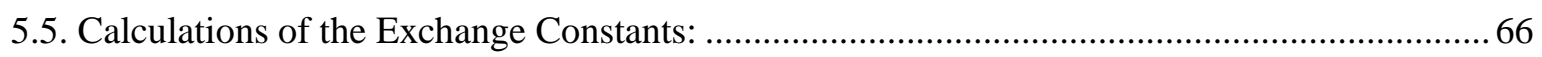

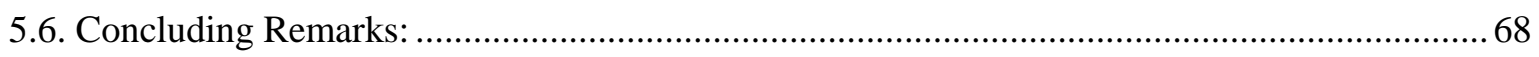

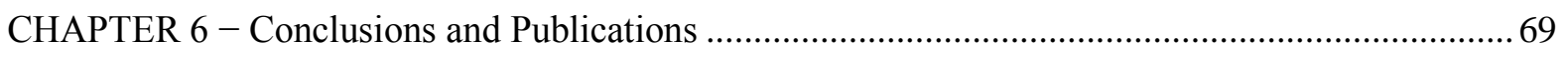

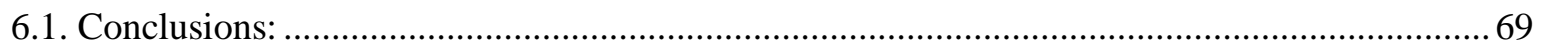

6.2. Publications of the Author Related to this Dissertation: ......................................................... 70

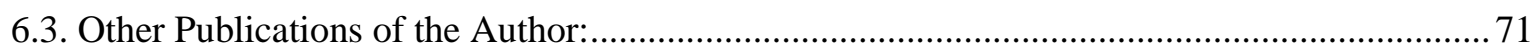

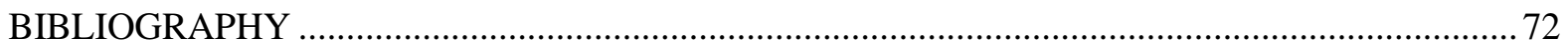




\section{LIST OF FIGURES}

Figure 1.1 Energy bandgap vs lattice constant for III-nitrides and III-phosphides. The bold lines indicate direct bandgap, whereas the dotted lines indicate indirect bandgap. Dashed lines are estimated values (reproduced from Ref. [2]) ........................ 2

Figure 1.2 Bandgap of III-nitrides as function of lattice constant compared to solar spectrum (Taken from Ref. [9])

Figure 1.3 Wurtzite structure of $\mathrm{GaN}$ with two different polarities (Taken from Ref. [3])........ 3

Figure 1.4 Schematic of $\mathrm{Al}_{\mathrm{x}} \mathrm{Ga}_{1-\mathrm{x}} \mathrm{N} / \mathrm{GaN}$ HEMT (From Ref. [10]) $\ldots \ldots \ldots \ldots \ldots \ldots \ldots \ldots \ldots \ldots \ldots$

Figure 1.5 The partial pressure of gases with molecular $\mathrm{M}=28 \mathrm{amu}$ as function of temperature for InN, AlN and GaN (Taken from Ref. [3])....

Figure 1.6 Approximate maximum operating temperatures of different piezoelectric materials (Reproduced from Ref. [14]).

Figure 1.7 Loss Spectrum of silica optical fiber (Taken from Ref. [16])................ 9

Figure 2.1 XPS survey scans of the AlN and AlN:Er films in the binding energy range of 0-700 $\mathrm{eV}$. The main peaks for the elements detected in the films are identified....

Figure 2.2 Oxygen atomic concentration in AlN:Er thin film as a function of Ar sputter time... 15

Figure 2.3 This figure shows Er (4d) peak before (black line), background (blue line) calculated by Shirley method and corrected peak after baseline subtraction (red line).

Figure 2.4 XPS plots of Al, N, O and Er peaks after 6 minutes of Argon ion sputtering of the AlN:Er thin film. Dots show the experimental data, black lines the peaks obtained after peak-fits for different chemical states and red line summation of the peaks for each element

Figure 2.5 X-ray diffraction patterns for the AlN and AlN:Er1 (1\% Er concentration in the target) thin films on Si substrate.

Figure 2.6 X-ray diffraction patterns of the two samples of $\mathrm{Er}_{2} \mathrm{O}_{3}$. For comparison, the expected line positions for $\mathrm{Er}_{2} \mathrm{O}_{3}$ along with Miller indices of a few prominent lines are shown at the top.

Figure 2.7 SEM image of cross-section of AlN:Er thin film........................... 19

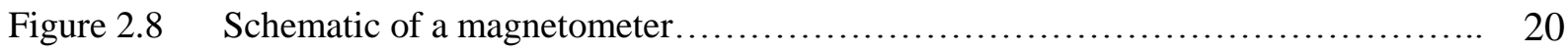


Figure 2.9 Schematic of optics in modified Mach-Zehnder interferometer in Polytec PSV-400 system (Taken from Ref. [33]).

Figure 2.10 Piezoelectric coefficient $\mathrm{d}_{33}$ for different AlN thin films as function of work functions of contact metals. The metals reported are $\mathrm{Al}, \mathrm{Ti}, \mathrm{Au}$ and $\mathrm{Pt}$ in order of increasing work function. (From Ref. [34])

Figure 2.11 Main steps involved in image reversal photolithography to deposit Pt metal contacts on AlN and AlN:Er thin films for piezoelectric coefficient measurements.

Figure 3.1 Stress components on the surface of an infinitesimal cube (Taken from Ref. [37]).....

Figure 3.2 (a) Unit cell showing the $\mathrm{a}_{1}, \mathrm{a}_{2}$, and c-axes. (b) Directions in Miller-Bravais indices. 28

(c) Relationship between $\mathrm{x}_{1}, \mathrm{x}_{2}$ and $\mathrm{x}_{3}$ axes in Fig. 3.1 and crystallographic directions. (Taken from Ref. [1]).

Figure 3.3 Refractive index of AlN:Er thin film as function of sputtering target power. The line connecting the data points is visual guide

Figure 3.4 AlN:Er displacement as function of ac frequency at $\mathrm{V}=40 \mathrm{~V}$. The lines connecting 33 the data points are for visual guide

Figure 3.5 Displacement as function of amplitude $\mathrm{V}$ of the ac signal....

Figure 3.6 Plots of $\mathrm{d}_{33}$ and refractive index for AlN:Er (3\%) films as function of sputtering target power. The lines connecting the data points are visual guides.

Figure 3.7 $\mathrm{d}_{33}$ vs thickness for AlN:Er1 and AlN thin films. The lines connecting the data points are visual guides

Figure $3.8 \mathrm{~d}_{33}$ vs thickness for AlN:Er3 and AlN thin films. The lines connecting the data points are visual guides

Figure $3.9 \mathrm{~d}_{33}$ vs thickness for AlN:Er4 and AlN thin films. The lines connecting the data points are visual guides

Figure 3.10 XRD pattern for un-annealed AlN and AlN:Er thin films.

Figure 3.11 XRD pattern for annealed AlN and AlN:Er thin films.

Figure 4.1: Temperature dependence of the magnetic susceptibility $\chi=\mathrm{M} / \mathrm{H}$ of Er metal in the temperature range of 2 to $300 \mathrm{~K}$ under ZFC and FC conditions.

Figure 4.2: $\quad$ Plots of computed $\mathrm{d}(\chi \mathrm{T}) / \mathrm{dT}$ from $\mathrm{ZFC}$ and $\mathrm{FC}$ data of Erbium metal to determine various transition temperatures when applied $\mathrm{H}=4000 \mathrm{Oe}$ 
Figure 4.3: Shown are the experimental results on the temperature dependence of the magnetic susceptibility $\mathrm{M} / \mathrm{H}$ for the Erbium metal with the solid line fit to the Curie-Weiss law (Eq. 4.16) with the parameters of the fit given in the figure

Figure 4.4: Magnetic Field dependence of the magnetization of the Erbium metal at $\mathrm{T}=5 \mathrm{~K} \ldots \ldots$

Figure 4.5: Magnetic Field dependence of the magnetization of the Erbium metal at $\mathrm{T}=50 \mathrm{~K}$ and $\mathrm{T}=110 \mathrm{~K}$. The lines connecting the data points are visual guides

Figure 4.6: Computed $\mathrm{dM} / \mathrm{dH}$ vs. $\mathrm{H}$ for the data of Fig 4.3 and Fig. 4.4 at $\mathrm{T}=5 \mathrm{~K}, 50 \mathrm{~K}$ and 110 K. .

Figure 4.7: Temperature dependence $(2 \mathrm{~K}$ to $300 \mathrm{~K})$ of the magnetic susceptibility $\chi=\mathrm{M} / \mathrm{H}$ for powder sample of $\mathrm{Er}_{2} \mathrm{O}_{3}$ under $\mathrm{ZFC}$ and $\mathrm{FC}$ conditions using $\mathrm{H}=100 \mathrm{Oe}$

Figure 4.8: Shown are the experimental results on the temperature dependence of the magnetic susceptibility M/H for the thin films of AIN and AlN:Er with the solid line fit to the Curie-Weiss law with the parameters given in the figure

Figure 4.9: $\quad \mathrm{M}$ vs $\mathrm{H}$ data (circles) for the AlN:Er thin film measured at $2 \mathrm{~K}$. Red line corresponds to fit to Eq. 3 with $\gamma_{1}=1.207 \gamma$ and blue line corresponds to $\gamma_{1}=\gamma$

Figure 5.1 Atomic model of bixbyite structure of $\mathrm{Er}_{2} \mathrm{O}_{3}$ (Modified from Ref. [64]). Red spheres are $\mathrm{O}$ atoms, blue and grey spheres are Er atoms at $\mathrm{C}_{3 \mathrm{i}}$ and $\mathrm{C}_{2}$ sites respectively.......

Figure 5.2 Magnetic ordering of $\mathrm{Er}^{3+}$ ions in the cubic unit cell of $\mathrm{Er}_{2} \mathrm{O}_{3}$ (reproduced from Moon et al. [55]) The unit cell has been expanded along the z-direction for clarity and the positions of the oxygen ions situated between the $\mathrm{Er}^{3+}$ ions are not shown.

Figure 5.3 Temperature dependence of the magnetic susceptibility of the two samples of $\mathrm{Er}_{2} \mathrm{O}_{3}$ for the zero-field-cooled and field-cooled cases in $\mathrm{H}=100$ Oe. The inset shows the expanded view of the data for $\mathrm{T}<6 \mathrm{~K}$

Figure 5.4 Magnetic field dependence of the magnetization of the $b-\mathrm{Er}_{2} \mathrm{O}_{3}$ and $n-\mathrm{Er}_{2} \mathrm{O}_{3}$ samples at $2 \mathrm{~K}$. Observed hysteresis of few Oe (inset) is within the experimental uncertainty of setting the field....

Figure 5.5 The plots of $\mathrm{M}$ vs. $1 / \mathrm{H}$ to estimate the saturation magnetization $\mathrm{M}_{\mathrm{S}}$ in the limit of $1 / \mathrm{H}=0$

Figure 5.6 Magnetic field dependence of the magnetization at several temperatures for $n-\mathrm{Er}_{2} \mathrm{O}_{3} . \quad 59$ For clarity, data for only a few select temperatures are shown 
Figure 5.7 Temperature dependence $\left(2 \mathrm{~K}\right.$ to $5 \mathrm{~K}$ ) of the magnetic susceptibility of $\mathrm{n}-\mathrm{Er}_{2} \mathrm{O}_{3}$ for 59 the zero-field-cooled case in different $\mathrm{H}$ from 100 Oe to $20 \mathrm{kOe}$

Figure 5.8 Plots of inverse susceptibility vs. temperature; solid lines fits to the Curie-Weiss law. 61

Figure 5.9 Plots of the computed $d(\chi \mathrm{T}) / d \mathrm{~T}$ vs. T obtained from the ZFC data near $\mathrm{T}_{\mathrm{N}}$ (Fig. 5.3) for the two samples of $\mathrm{Er}_{2} \mathrm{O}_{3}$. Top panel shows the specific heat vs. temperature data of Tang et al. [62]

Figure 5.10 Data of Fig.5.6 are used to compute and plot $d(\chi \mathrm{T}) / d \mathrm{~T}$ vs. T curves for determining $\mathrm{T}_{\mathrm{N}}$ for different $\mathrm{H}$. The inset shows the variation of $\mathrm{T}_{\mathrm{N}} \mathrm{vs}$. $\mathrm{H}^{2}$ to determine $\mathrm{D}_{1}$ of Eq. (5.1)

Figure 5.11 Computed $d \mathrm{M} / d \mathrm{H}$ vs. $\mathrm{H}$ for the data of Fig. 5 at $\mathrm{T}=2.0 \mathrm{~K}$ and 3.3 K. Dashed lines

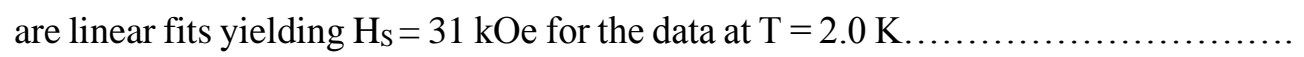

Figure 5.12 Plots of $\mathrm{dM} / \mathrm{dH}$ vs. $\mathrm{H}$ computed from the data of $\mathrm{M}$ vs. $\mathrm{H}$ of Fig. 5.6 in the range of 2.0-4.0 K taken at $0.1 \mathrm{~K}$ steps. The dashed lines connect the crests depicting the spinflop field $\mathrm{H}_{\mathrm{SF}}$ and its temperature dependence.............................. 64

Figure 5.13 Variation of reduced spin-flop field $h=\mathrm{H}_{\mathrm{SF}}(\mathrm{T}) / \mathrm{H}_{\mathrm{SF}}(0)$ vs. the reduced Nèel temperature $\mathrm{t}=\mathrm{T} / \mathrm{T}_{\mathrm{N}}$. The solid line is based on the solution to Eq. (5.2) 


\section{CHAPTER 1 - Introduction and Background}

\subsection{Introduction:}

In this chapter, properties and applications of Group III-nitrides and rare-earth doped Group III-nitrides in general and Aluminum Nitride (AIN) and erbium doped AlN (AlN:Er) in particular are described. Based on the properties and applications of these materials, the motivation behind the study of properties of AlN:Er is outlined.

\subsection{Group III-Nitrides:}

Group III-nitride materials AlN, GaN and InN and their alloys AlGaN, AlInN and InGaN possess properties that are considered very important for device applications. The most important aspect of the properties of III-nitrides is that it is the only class of materials using suitable combination of which we can engineer the systems with direct bandgap of $0.7 \mathrm{eV}$ to $6.2 \mathrm{eV}$ [2-4]. The emission from these materials covers the emission spectra from deep UV to infrared wavelengths and includes all the visible region. No other class of materials possess this property. Fig. 1 shows the relationship between the lattice constant and energy bandgap for III-nitrides and III-phosphides (the other class of materials with emission wavelength in visible region) and it can be seen from the figure that bandgap of only IIInitrides cover the full visible region of $1.8 \mathrm{eV}$ to $2.8 \mathrm{eV}$ [3]. This property of III-nitrides leads to their applications as emitters, detectors and photovoltaics [4].

Due to the emission wavelengths Group III-nitrides cover, there has been considerable focus to develop light emitting diodes (LEDs) in UV and visible region. In the visible region the focus is to improve the efficiency of solid state lighting (SSL) to replace the inefficient incandescent and compact fluorescent lamps. In the US, for SSL the DOE has the target to achieve the efficiency of $232 \mathrm{~lm} / \mathrm{W}$ by year 2020, which by 2014 is $133 \mathrm{~lm} / \mathrm{W}$ [5]. The white light for SSL is produced using either the suitable combination of red, green and blue LEDs or by converting the light from any color of LED to pass through phosphor. As of 2014, the power efficiency of blue, green and red LEDs is 55\%, $22 \%$ and $44 \%$ respectively and to achieve the target of efficiency of $232 \mathrm{~lm} / \mathrm{W}$, the targeted power efficiencies of blue, green and red LEDs need to be $80 \%, 35 \%$ and $55 \%$ respectively. The overall efficiency of color mixed LEDs is affected greatly by low efficiency of green LEDs and to achieve the target efficiency, Group III-nitride based LEDs will remain the focus of research in this field.

Similar to the visible region, Group III-nitride emission covers the UV wavelength region and have been successfully used to fabricate LEDs and detectors in this region [6,7]. These devices can provide alternative to toxic and environment unfriendly gas and mercury based UV light sources. These 
light sources have potential applications as disinfectant, UV polymer curing, smaller wavelength light sources for improved UV photolithography and high density optical data storage [6, 8]. The Group IIInitride materials with bandgap corresponding to UV region are also used as detectors in applications where UV light detectors blind to solar light are needed [9]. These detectors replace traditionally used photomultiplier tubes which need vacuum tubes and high voltages or $\mathrm{Si}$.

As shown in figure 1.2, the bandgaps of $\mathrm{InN}$ and $\mathrm{InGaN}$ cover the full solar spectrum [10]. This makes InGaN a suitable material for solar cell applications. Also InN and InGaN show better radiation hardness compared to other materials used for solar cell applications. Solar cells are widely used in space applications but the high energy radiations present in outer space easily degrade the traditional solar cells which are based on $\mathrm{Si}, \mathrm{GaAs}$ or GaInP. The higher resistance to high energy radiation makes $\mathrm{InGaN}$ an attractive choice for solar cell applications.

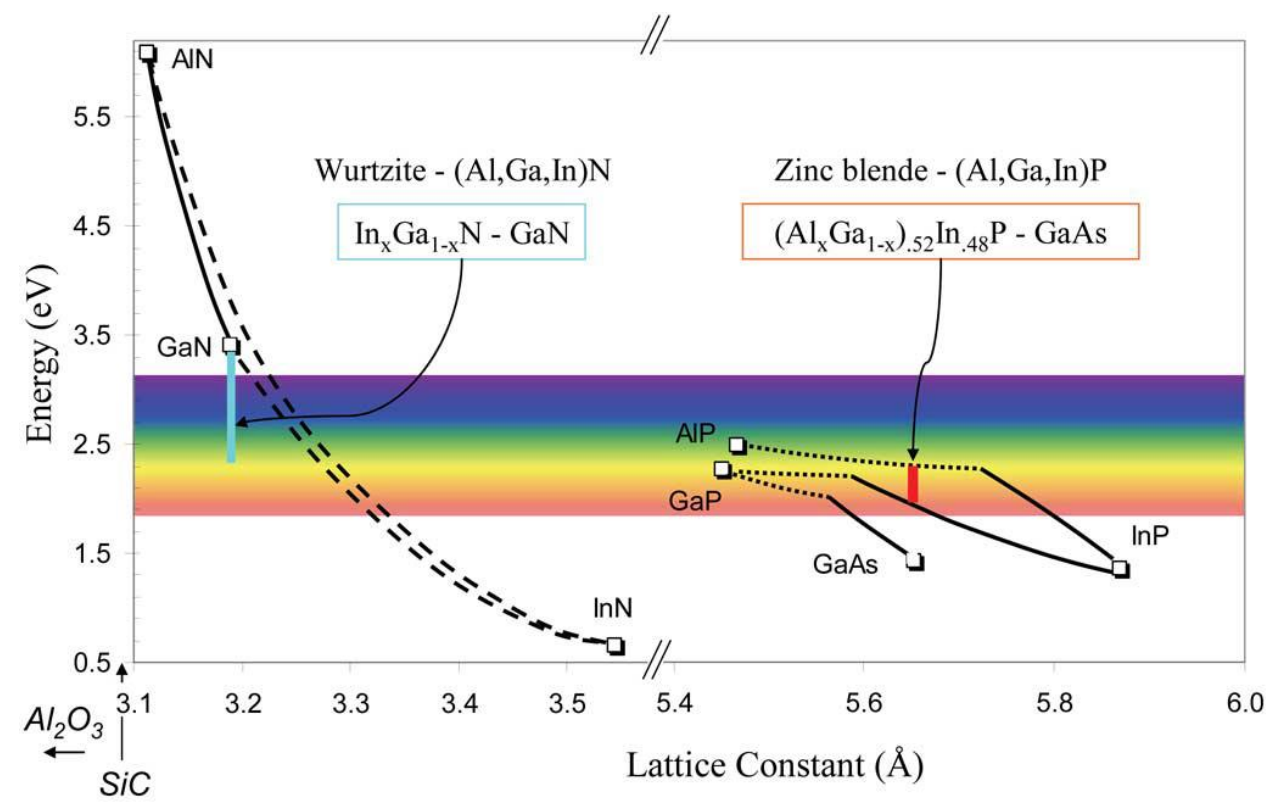

Figure 1.1: Energy bandgap vs lattice constant for III-nitrides and III-phosphides. The bold lines indicate direct bandgap, whereas the dotted lines indicate indirect bandgap. Dashed lines are estimated values (reproduced from Ref. [3]). 


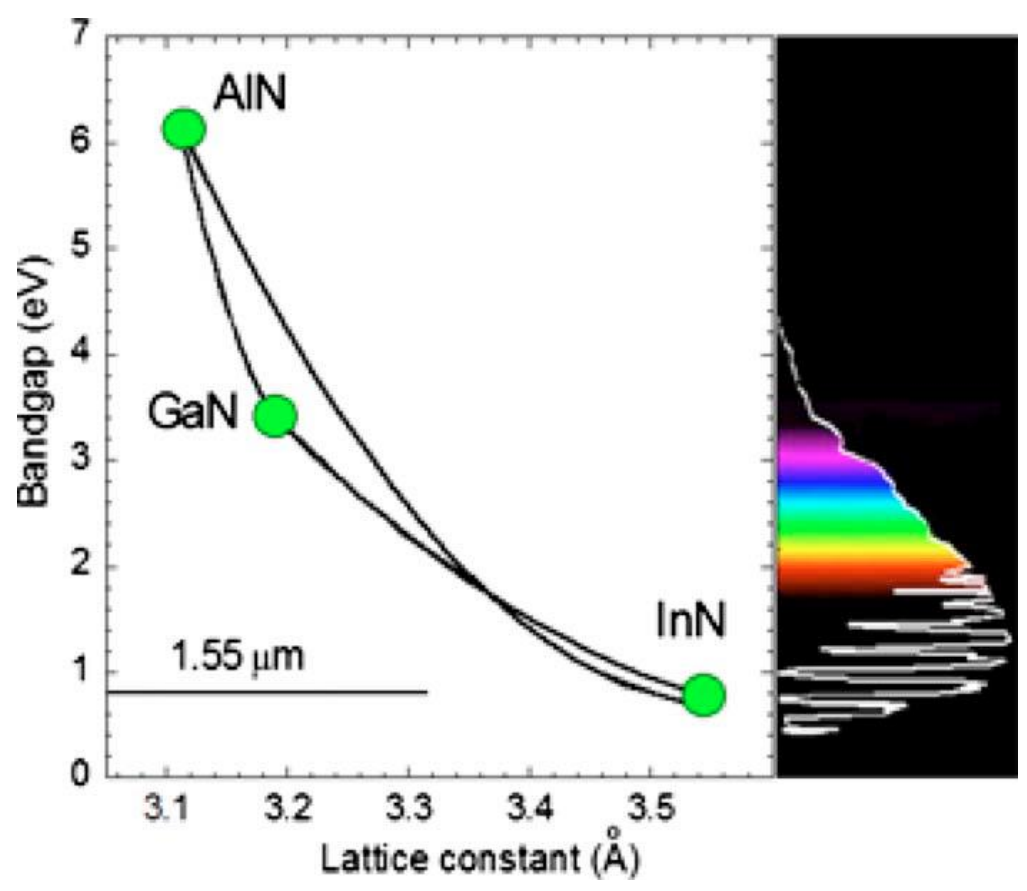

Figure 1.2: Bandgap of III-nitrides as function of lattice constant compared to solar spectrum (from Ref. [10]).

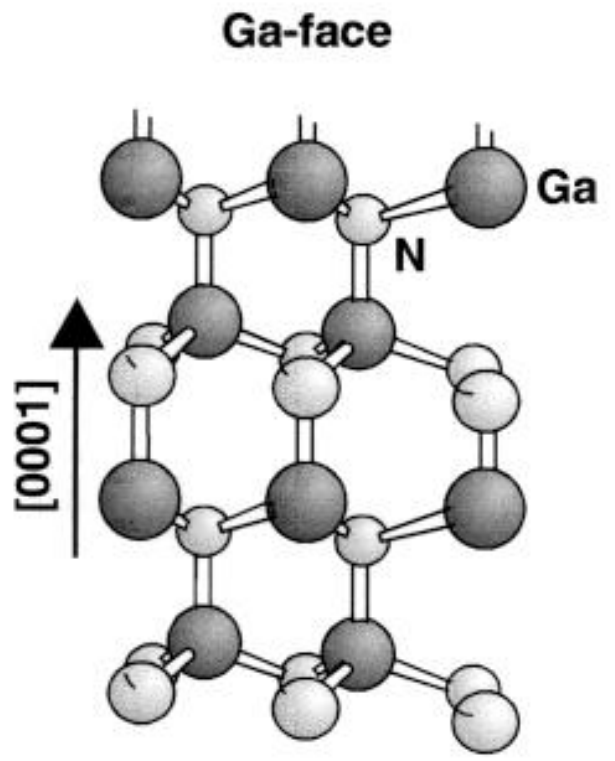

Substrate

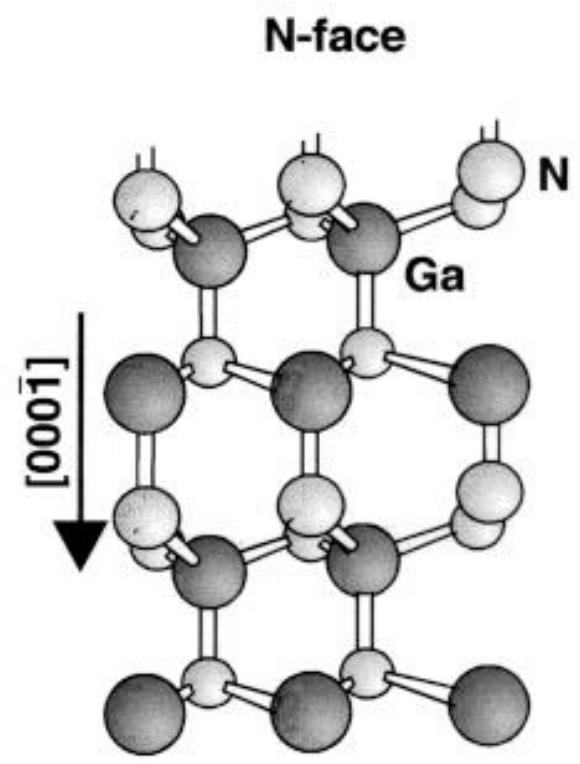

Substrate

Figure 1.3: Wurtzite structure of GaN with two different polarities (Taken from Ref. [4]). 
Group III-nitrides have non-centrosymmetric wurtzite crystal structure which exhibits crystallographic polarity in the c-axis direction (as shown in Fig. 1.3) [4]. Group III-nitride also have strain or piezoelectric induced polarity. Crystallographic polarization, also known as spontaneous polarization, is also the highest for AlN among all III-nitrides and their alloys. The total polarization of III-nitride is sum of the spontaneous polarization $\left(\mathrm{P}_{\mathrm{SP}}\right)$ and piezoelectric induced polarization $\left(\mathrm{P}_{\mathrm{PE}}\right)$. When there is heterojunction of two different nitrides such as GaN and AlGaN, it results in a polarization gradient at the junction leading to charge density $\sigma$ at the junction. As the spontaneous polarization is larger for $\mathrm{AlGaN}$, it leads to positive charge density which is compensated by a flow of free electrons in a sheet region at the junction. This electron flow is known as two-dimensional electron gas flow (2DEG).

Transistors based on 2DEG provide an alternative to the conventional transistors where flow of electrons is facilitated by doping the active region, which due to the collisions of electrons with the dopants, leads to low mobility of the charge carriers. A typical 2DEG based transistor is shown in Fig. 1.4 and is known as High Electron Mobility Transistor (HEMT) [11]; these are finding applications in high power, high frequency electronics. Combined with the radiation hardness of Group III-nitrides as described above, GaN/AlGaN based HEMT can be used in electronics in space applications where conventionally used $\mathrm{Si}$ based electronics is damaged by high energy radiation and needs external shielding to operate in this environment [12].
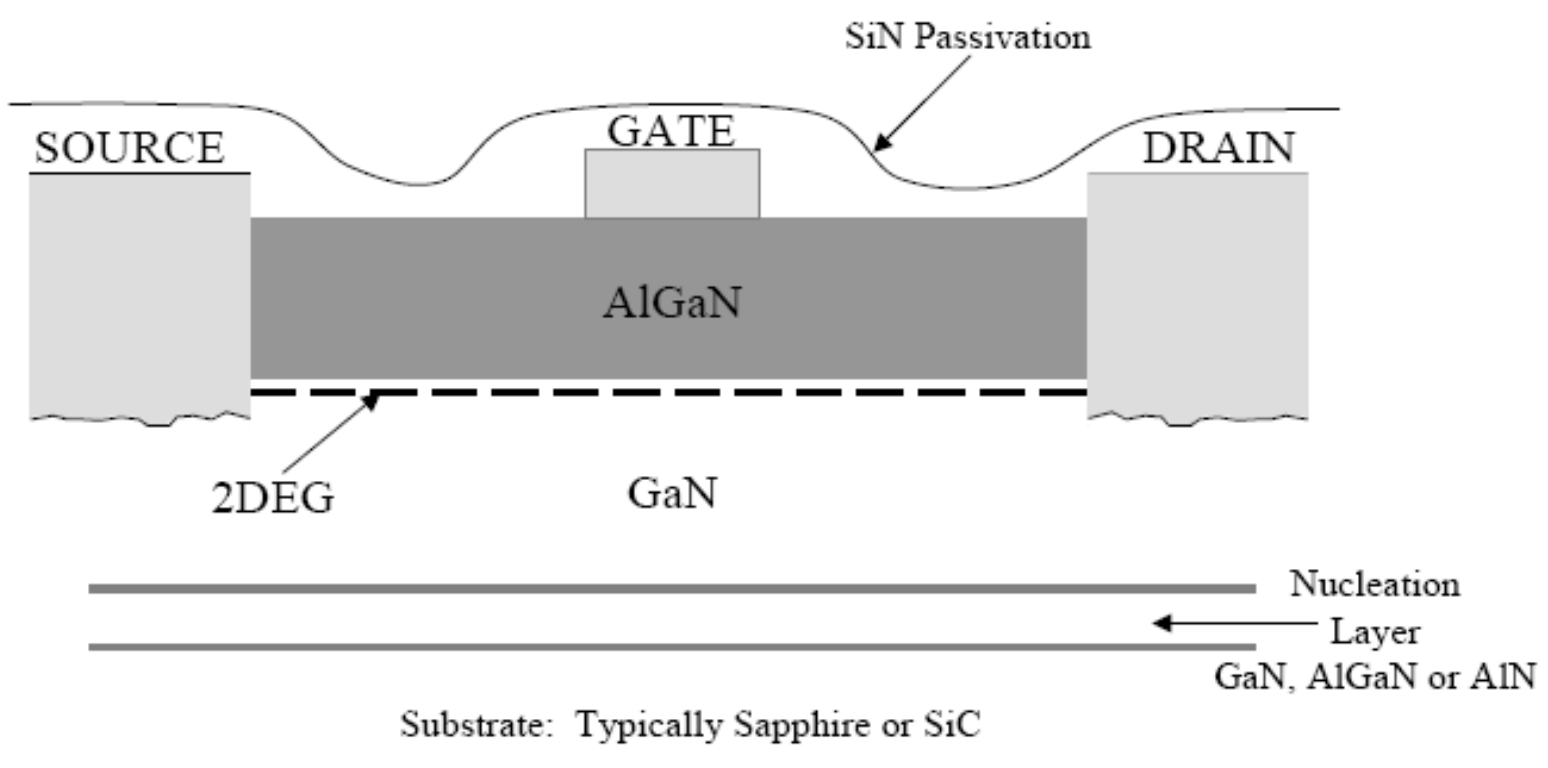

Figure 1.4: Schematic of $\mathrm{Al}_{\mathrm{x}} \mathrm{Ga}_{1-\mathrm{x}} \mathrm{N} / \mathrm{GaN}$ HEMT (From Ref. [11]). 
The polarization of the 2DEG is influenced by many external factors. These changes in polarization in turn can be used to detect the source of such factors. Examples of such properties are: (i) Sensing the gases and biomolecules based on their effect on the surface polarization; (ii) the piezoelectric induced polarization is affected by the strain in the heterojunction and the sensor based on this can be used to sense pressure or strain; and (iii) the polarization is affected by electromagnetic radiation and magnetic field and it can be used for Hall and UV sensors respectively [4].

Other than optoelectronic and electronic applications, Group III-nitride materials possess properties which find applications in Micro Electro Mechanical Systems (MEMS) and Nano Electro Mechanical Systems (NEMS). Devices based on MEMS can sense the changes in the surrounding environment and then can react to these changes using micro-sensors and actuators contained in these devices [13]. Most of the current integrated electronics is based on Si and micro and nanofabrication techniques for Si based electronics are well developed. So MEMS are preferred choice. But Si based devices have limitations such as loss of mechanical stability at temperatures above $500{ }^{\circ} \mathrm{C}$, no resistance to corrosive environments and non-compatibility of Si to bio-molecules. This leads to situations where alternatives to Si based MEMS are required. Wide bandgap semiconductors (both SiC and III-nitrides) have properties such as high mechanical strength, and thermal, chemical and biochemical stability, thus providing the needed alternatives.

Some of the properties which make Group III-nitrides as suitable materials for MEMS are their high piezoelectric coefficients, high in-plane acoustic velocities, high electric, thermal and mechanical strengths and high heat conductance [13]. Some of the reported piezoelectric coefficients of AlN and GaN are shown in Table 1.1.

\begin{tabular}{|l|l|l|l|}
\hline Material & Crystallinity & Substrate & $\mathrm{d}_{33}(\mathrm{pm} / \mathrm{V})$ \\
\hline GaN & Single & SiC & 3.7 \\
\hline GaN & Single & AlN/SiC & 3.9 \\
\hline GaN & Poly & Si & 2.6 \\
\hline AlN & Single & & 4.53 \\
\hline AlN & Poly & Sapphire & 5.53 \\
\hline AlN & Poly & Si & 5.4 \\
\hline
\end{tabular}


Table 1.1: $d_{33}$ coefficients reported for AlN and GaN (Data from Ref. [13]).

Other commonly used materials with high piezoelectric coefficients are $\mathrm{PbZr}_{0.6} \mathrm{Ti}_{0.4} \mathrm{O}_{3}(\mathrm{PZT})$ with $\mathrm{d}_{33}=374 \mathrm{pm} / \mathrm{V}, \mathrm{BaZiO}_{3}$ with $\mathrm{d}_{33}=82 \mathrm{pm} / \mathrm{V}$, and $\mathrm{BaPbTiO}_{3}$ with $\mathrm{d}_{33}=120 \mathrm{pm} / \mathrm{V}$ [13]. These materials are ferroelectric ceramics in nature and Group III-nitrides have much lower piezoelectric coefficients than these materials. However, these materials are incompatible with the Si based technology and are known to degrade the functionality of Si based devices by $\mathrm{Zn}$ or $\mathrm{Pb}$ contamination. In addition these oxide ceramics possess low biocompatibility compared to Group III-nitrides. Also, ferroelectric piezoelectric materials lose their piezoelectric properties at temperatures above their Curie temperatures. Curie temperatures for $\mathrm{PZT}, \mathrm{BaZiO}_{3}$ and $\mathrm{BaPbTiO}_{3}$ are $365{ }^{\circ} \mathrm{C}, 120{ }^{\circ} \mathrm{C}$ and $150{ }^{\circ} \mathrm{C}$ respectively. On the other hand, AlN is reported to maintain its piezoelectric properties up to $1150{ }^{\circ} \mathrm{C}$. Therefore in cases where functional layers in devices are required to operate at high temperatures, Group III-nitrides are the preferable choice. In addition to this, as the thickness of the III-nitrides can be controlled to monolayer scale, the precise control on the properties of functional layers based on IIInitrides can be achieved.

$\mathrm{ZnO}$ is the other material which is deposited as thin film and has $\mathrm{d}_{33}=12.3 \mathrm{pm} / \mathrm{V}$. This is higher than the reported values for AlN but AlN has higher acoustic velocity as compared to $\mathrm{ZnO}$, which leads to operation of AlN based devices at higher frequencies. Also, AlN has better electrical, mechanical and thermal properties which makes AlN better choice for devices operating at higher temperature with less insertion loss and aging [14]. These properties make Group III-nitrides useful as piezoelectric functional layers in MEMS devices.

\subsection{Importance of Aluminum Nitride:}

Among the Group III-nitrides, AlN has some properties that make it especially useful in device applications. First, it has the highest reported piezoelectric coefficient and highest acoustic velocity among the III-nitrides [13]. Therefore, in Group III-nitride based MEMS, AlN is used as functional layer. AlN is also thermally most stable among Group III-nitrides. Melting points of Group III-nitrides are much higher than their decomposition temperature [4]. As the temperature is increased, $\mathrm{N}_{2}$ desorption takes place. The thermal stability of the III-nitrides is measured in terms of temperature at which nitrogen desorption takes place. Figure 1.5 shows the partial pressure of the gases with mass 28 amu $\left(\mathrm{N}_{2}{ }^{+}, \mathrm{CO}^{+}, \mathrm{C}_{2} \mathrm{H}_{4}^{+}\right)$as the temperature increases. As is clear from the figure, the exponential increase in partial pressure of $\mathrm{N}_{2}$ takes place at temperatures $630{ }^{\circ} \mathrm{C}, 850{ }^{\circ} \mathrm{C}$ and $1040{ }^{\circ} \mathrm{C}$ for $\mathrm{InN}, \mathrm{GaN}$ and AlN respectively. Therefore AIN is most suitable for applications at high temperatures. Desorption of nitrogen at higher temperature is also important in cases where it can adversely affect the properties of adjacent layers in a device. AlN also maintains piezoelectric properties at high temperatures. Turner 
et al. [15] reported the piezoelectric response up to $1150{ }^{\circ} \mathrm{C}$. Figure 1.6 shows the comparison of maximum operation temperature for different piezoelectric materials and AlN has the highest value [15].

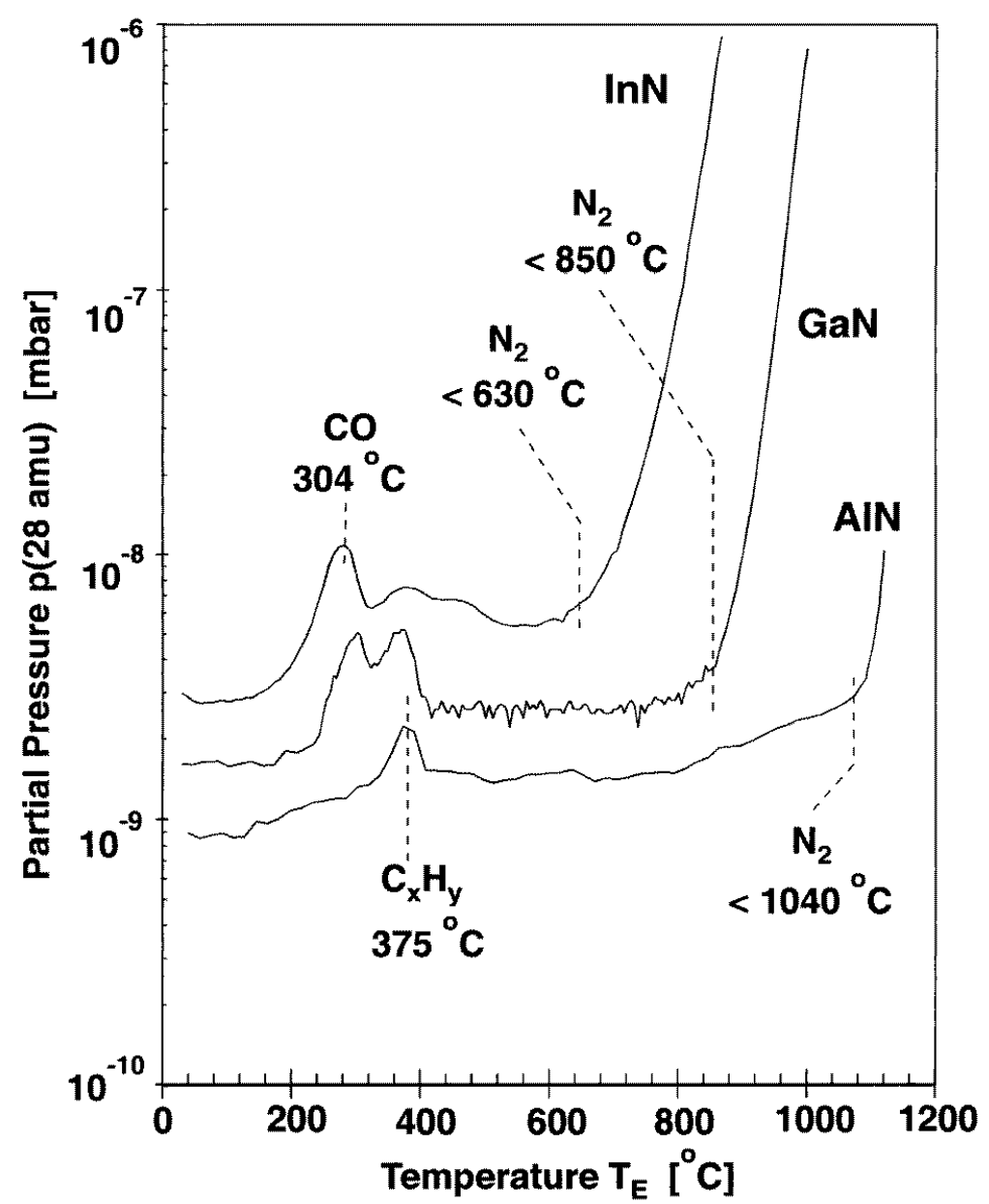

Figure 1.5: The partial pressure of gases with molecular $\mathrm{M}=28 \mathrm{amu}$ as function of temperature for InN, AlN and GaN (Taken from Ref. [4]).

From Table 1.1, it is clear that piezoelectric coefficient $d_{33}$ for polycrystalline AlN is very close to single crystalline AlN. Among III-nitrides, AlN is most widely deposited in polycrystalline form using reactive sputtering [13], while other III-nitrides are grown epitaxially using techniques such as Metal Organic Chemical Vapor Deposition (MOCVD), Molecular Beam Epitaxy (MBE) and Pulsed Laser Ablation (PLA) methods, where typically required temperature is $900{ }^{\circ} \mathrm{C}$ to $1100{ }^{\circ} \mathrm{C}[4]$. On the other hand, polycrystalline AlN can be deposited at temperatures close to $400^{\circ} \mathrm{C}$ [16]. As discussed earlier, AlN is needed for many applications in MEMS where AlN is needed to be integrated with other devices and deposited on surfaces such as metal contacts. Typical temperature of such device 
fabrication is below $500{ }^{\circ} \mathrm{C}$ [16], thus avoiding melting of the metal contacts and degrading other components of the device.

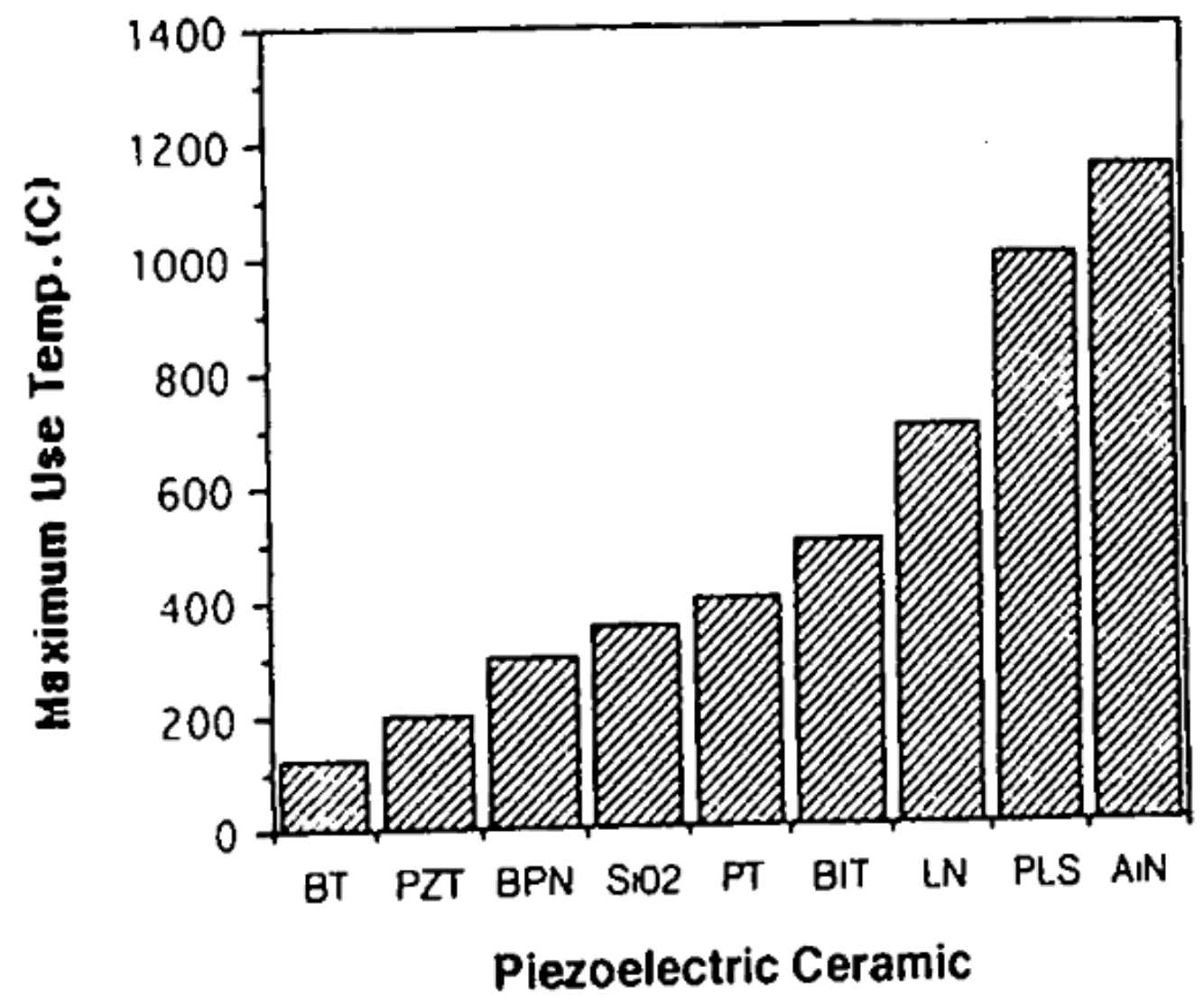

Figure 1.6: Approximate maximum operating temperatures of different piezoelectric materials (Reproduced from Ref. [15]).

\subsection{Rare-earth Doped Materials and AIN:Er:}

Lanthanide elements with $4 \mathrm{f}$ electrons, starting from Lanthanum to Ytterbium are called rare earths. In rare earths, partially filled $4 \mathrm{f}$ orbitals are surrounded by filled $5 \mathrm{~s}^{2}$ and $5 \mathrm{p}^{6}$ orbitals. Since $4 \mathrm{f}$ electrons in partially filled orbitals are shielded by filled $5 \mathrm{~s}$ and $5 \mathrm{p}$ orbitals, electronic levels of rare earth elements in solids are largely unaffected by surrounding electric fields of other ions [17]. Intra $4 \mathrm{f}$ transitions in free ions are parity forbidden. However, when rare earth elements are present in a host material, intra $4 \mathrm{f}$ transitions become allowed due to mixtures of states of different parities. These transitions are observed to be narrow and intense. Different rare earth elements have different emission wavelengths ranging from visible spectra to infrared region. Depending on the requirement of an application, different rare earth doped 
materials (acting as hosts) are widely used in optoelectronics. Some of the technological wavelengths of different rare earths are described in review article by Kenyon et al [17]. For $\mathrm{Er}^{3+}$ ions, the emission wavelength of ${ }^{4} \mathrm{I}_{13 / 2} \rightarrow{ }^{4} \mathrm{I}_{15 / 2}$ transition is $1535 \mathrm{~nm}$. For telecommunication applications, silica optical fibers are extensively used. Figure 1.7 shows the optical loss spectrum for silica optical fiber and it is clear from this that minimum loss occurs in 1530-1560 nm wavelength range, which coincides with the emission from $\mathrm{Er}^{3+}$ ions. Due to this, Er doped materials are of great technological interest to provide integrated optical sources and amplifiers at wavelengths needed for telecommunication technology.

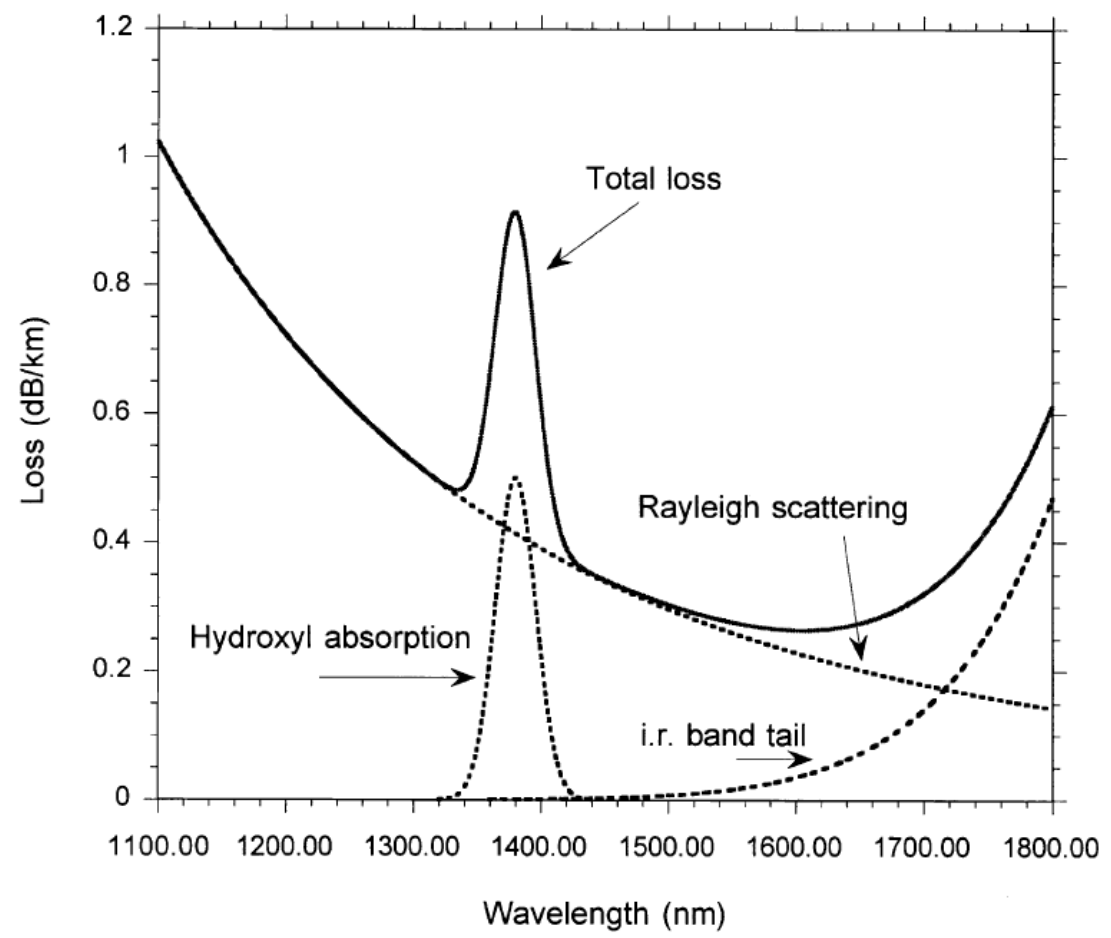

Figure 1.7: Loss Spectrum of silica optical fiber (Taken from Ref. [17]).

Apart from the $1530 \mathrm{~nm}$ wavelength of Er emission, Er, Eu, Tb and Ce ions have been used in CRT phosphors to produces RGB (red, green and blue) lights for different color displays. Favennec et al. [18] showed that the temperature dependent variation of intensity of rare earth ion emission depends inversely on the band gap of the host material. Hence wide band gap group III-nitrides are natural choice as rare earth hosts for applications ranging from visible spectrum for LEDs to infrared region for telecommunication applications. 
Both visible and infrared emission have been observed by different groups in $\mathrm{GaN}$ and AlN hosts [19, 20]. AlN has the highest bandgap among III-nitrides and hence has least temperature dependent attenuation. In addition to this, it is comparatively easier to deposit Er doped AlN using the magnetron sputtering [21].

In addition to the optoelectronic properties, rare earth doping has been reported to improve the piezoelectric properties of the doped materials [22, 23]. As mentioned in previous sections, among III-nitrides AlN has the highest piezoelectric response and is thermally most stable. Therefore motivated by the fact that $\mathrm{Er}$ is the most important rare earth dopant for technological applications and AIN is the most suitable host, piezoelectric, structural and magnetic properties of the AlN:Er thin films were investigated in this work. These results and their discussion and interpretation are presented in this dissertation.

\subsection{Organization of the Dissertation:}

The rest of the dissertation is organized as follows: In Chapter 2, methodology for the sample preparation of AlN and AlN:Er thin films using DC-Magnetron sputter station is described along with various techniques used to characterize the thin films and other related materials. Since details of the experimental techniques are extensively available in the literature, only brief overviews of the techniques is given. In Chapter 3, a brief theory of piezoelectricity is given and it is followed by the experimental results on the piezoelectric and structural properties of AlN and AlN:Er thin films. The improved piezoelectric response of $\mathrm{AlN}$ :Er in comparison to AlN is explained on the basis of reported structural properties of the thin films. In Chapter 4, after providing a brief theory of magnetism, results obtained from the magnetic measurements on AlN and AlN:Er thin films are reported. Based on the magnetic properties of the AlN:Er thin films, the electronic state and location of Er is determined. The other two possibilities of Er being present in the AlN:Er thin films were, $\operatorname{Er}_{2} \mathrm{O}_{3}$ or free $\mathrm{Er}$. To rule out these possibilities, magnetic measurements were carried out on commercially obtained $\mathrm{Er}_{2} \mathrm{O}_{3}$ powder and Er metal samples; these results are also reported in Chapter 4. The more detailed experimental results on $\mathrm{Er}_{2} \mathrm{O}_{3}$ samples are presented in Chapter 5 along with analysis of these results to explain the complex magnetic structure of $\mathrm{Er}_{2} \mathrm{O}_{3}$ and its magnetic field induced transitions. The experimental results presented in Chapters 3, 4 and 5 along with their analysis and discussions are the important new contributions of this dissertation. The major 
conclusions resulting from this work along with the list of publications based on this research are given in Chapter 6. 


\section{CHAPTER 2 - Sample Preparation and Experimental Procedures}

\subsection{Introduction:}

In this chapter, experimental procedures used for preparing the samples are described and relevant details of various analytical techniques employed in characterizing the properties of the samples are given. The relevant samples are thin film samples of AlN and AlN:Er and commercial samples of $\mathrm{Er}_{2} \mathrm{O}_{3}$ and Er metal.

\subsection{Synthesis of AIN and AIN:Er Films:}

For the deposition of Aluminum Nitride (AlN) several techniques such as Metal Organic Chemical Vapor Deposition (MOCVD), Molecular Beam Epitaxy (MBE), electron cyclotron resonance dual-ion-beam sputtering and pulsed laser ablation have been reported in the literature [16]. These methods produce better crystalline quality AlN thin films but all these methods of AIN deposition require high temperatures ranging from $900{ }^{\circ} \mathrm{C}$ to $1200{ }^{\circ} \mathrm{C}$. For microelectromechanical systems, piezoelectric thin films need to be deposited, maintaining the temperature compatible with integrated circuit technology, which should not exceed $500{ }^{\circ} \mathrm{C}$. The desired polycrystalline films of AlN can be deposited using reactive DC-magnetron sputtering while maintaining the temperature below $500{ }^{\circ} \mathrm{C}$. In this chapter, experimental procedures to deposit the AlN and AlN:Er thin films using DC-magnetron sputtering and their structural and chemical characterization are described. Both set of films were deposited on double side polished Si (100) substrate with resistivity of 1-20 $\Omega$-cm, using the DCmagnetron reactive sputtering.

Samples of Er-free AlN films were reactively sputtered using CVC 610 dc-magnetron sputtering station. The target used for AlN deposition was 2-inch diameter 99.999\% Al disk, purchased from Kurt J. Lesker (Part number EJTALXX502A4). For preparing the AlN: Er films, 2-inch diameter disk alloy of 97\% $\mathrm{Al}$ and 3\% Er, purchased from Kurt J. Lesker (Part No. EJTALERSPL03) was used. In comparison to nitrogen, oxygen has much higher affinity towards aluminum [24]. Therefore any oxygen present in the sputtering chamber gets incorporated into the AlN thin films. Oxygen is known to adversely affect the piezoelectric properties, thermal conductivity and optical properties of the AlN thin films [25-27]. Therefore it is important to reduce the oxygen level as low as possible in the sputtering chamber. To achieve this, before sputtering, the base pressure of $2.0 \times 10^{-6} \mathrm{mbar}$ was attained using a CTI-8 cryo-pump. To outgas the chamber while pumping down, it was heated to $120{ }^{\circ} \mathrm{C}$ for two hours. To remove oxygen and other contaminations from the targets, pre-sputter for 10 minutes 
was performed in argon (30 sccm flow) environment and a target power of 200W. After the targets were cleaned, high purity $\mathrm{N}_{2}$ and $\mathrm{Ar}$ were introduced at the flow rate of $27.0 \mathrm{sccm}$ and $3.0 \mathrm{sccm}$ respectively and pre-sputter was performed for 5 minutes at chamber pressure of 45 mbar to poison the targets before the actual deposition is started.

\subsection{Erbium Oxide (Er2O3) Powder:}

As described is next section, there is significant amount of oxygen present in the AlN:Er thin films. Therefore the possibility of Er present as $\mathrm{Er}_{2} \mathrm{O}_{3}$ has been considered. One possibility of $\mathrm{Er}$ and $\mathrm{O}$ present as $\mathrm{Er}_{2} \mathrm{O}_{3}$ was proposed. To verify that possibility, commercially available $\mathrm{Er}_{2} \mathrm{O}_{3}$ powder samples were studied for their structural and magnetic properties. Measurements were carried out on two different samples of $\mathrm{Er}_{2} \mathrm{O}_{3}$ : (i) bulk $\mathrm{Er}_{2} \mathrm{O}_{3}$ powder sample purchased from Alfa-Aesar (stock \# 11309) labelled here as b- $\mathrm{Er}_{2} \mathrm{O}_{3}$ and (ii) nanocrystalline sample with average particle size of $43 \mathrm{~nm}$ purchased from Nanostructured and Amorphous Materials Inc. (stock \# 2310ZQ) labelled here as n$\mathrm{Er}_{2} \mathrm{O}_{3}$. These samples were used as received without any modifications or purification.

\subsection{X-ray Photoelectron Spectroscopy (XPS):}

To determine the elemental composition of the thin films, XPS measurements on the AlN and AlN:Er thin films were performed using Physical Electronics PHI 5000 VersaProbe X-ray Photoelectron microscope with an $\mathrm{Al} \mathrm{K}_{\alpha} \mathrm{X}$-ray source $(1486.6 \mathrm{eV})$ operated at $25 \mathrm{~W}$ and $15 \mathrm{kV}$. The films were sputtered with Ar ions to do the depth measurements and the alignment to the adventitious carbon (1s) peak with binding energy $286.8 \mathrm{eV}$ [28] was made at surface and to $\operatorname{Ar} 2 \mathrm{p}_{3 / 2}$ [29] after argon ion sputtering. On the thin films, survey scans were performed from binding energy ranging from $0 \mathrm{eV}$ to $700 \mathrm{eV}$. This range covers all the core levels for the elements of $\mathrm{Al}, \mathrm{N}, \mathrm{Er}$ and $\mathrm{O}$ in this region. Figure 2.1 shows the survey scans for AlN and AlN:Er films. The presence of Er in the films is evident from the $\mathrm{Er}(4 \mathrm{~d})$ peak at the binding energy of around $168.8 \mathrm{eV}$. Similar survey scan was performed after sputtering with argon ion and it too showed the presence of Er, but with reduced intensity for the $\mathrm{O} 1 \mathrm{~s}$ peak, which is consistent with the fact that AlN film surface oxidizes in atmosphere. Figure 2.2 shows the oxygen atomic concentration in AlN:Er thin film as function of the depth sputtering time. It is clear from the figure that AlN:Er film is highly oxidized and away from the surface the oxygen concentration decreases as the film is sputtered with Ar. Along with survey scans, detailed multiplex scans are performed for the binding energies of interest. The obtained peaks were fitted with Pseudo-Voigt curves following Rosenberg et al. [30] to calculate the area under the curves and peak binding energies.

Before the area calculations, the baselines for the peaks are determined by integrated background method [31]. Figure 2.3 shows the multiplex scan obtained for AlN:Er thin film for the 
binding energy range corresponding to $\operatorname{Er}(4 \mathrm{~d})$ peak, baseline determination and corrected Er (4d) peak. For the Shirley Method of baseline calculation, inbuilt feature of OriginLab Software is used.

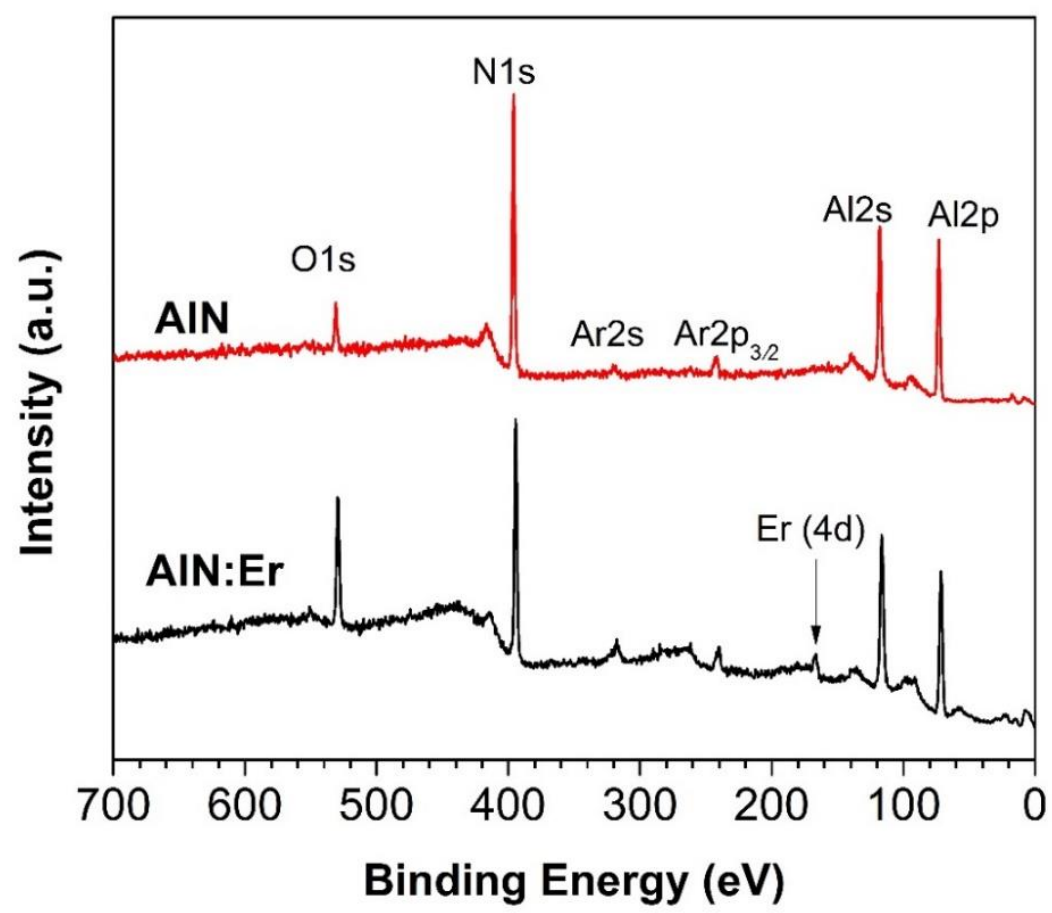

Figure 2.1: XPS survey scans of the AlN and AlN:Er films in the binding energy range of 0$700 \mathrm{eV}$. The main peaks for the elements detected in the films are identified.

XPS signal for a given energy is shown in figure 2.3. Similar treatment is applied to binding energies corresponding to $\mathrm{Al}(2 \mathrm{~s}), \mathrm{N}(1 \mathrm{~s})$ and $\mathrm{O}(1 \mathrm{~s})$ peaks. Figure 2.4 shows the multiplex scans for $\mathrm{Er}, \mathrm{Al}, \mathrm{O}$ and $\mathrm{N}$ after the Shirley background correction. The areas under the peaks are used to determine the percentage content of each element in the thin films. For this, the atomic sensitivity factors provided by the equipment manufacturer were used. If the sensitivity factor of an element $A$ is $S_{A}$ and $N_{A}$ is the area for peak corresponding to this peak, then fraction of atomic concentration is given by the equation:

$$
x_{A}=\frac{N_{A} / S_{A}}{\sum_{i}^{n}\left(N_{i} / S_{i}\right)}
$$

The area sensitivity factor used for the four elements viz. Aluminum, Nitrogen, Erbium and Oxygen as provided by the manufacturer of the XPS instrument are $0.256,0.499,2.65$ and 0.733 respectively. 


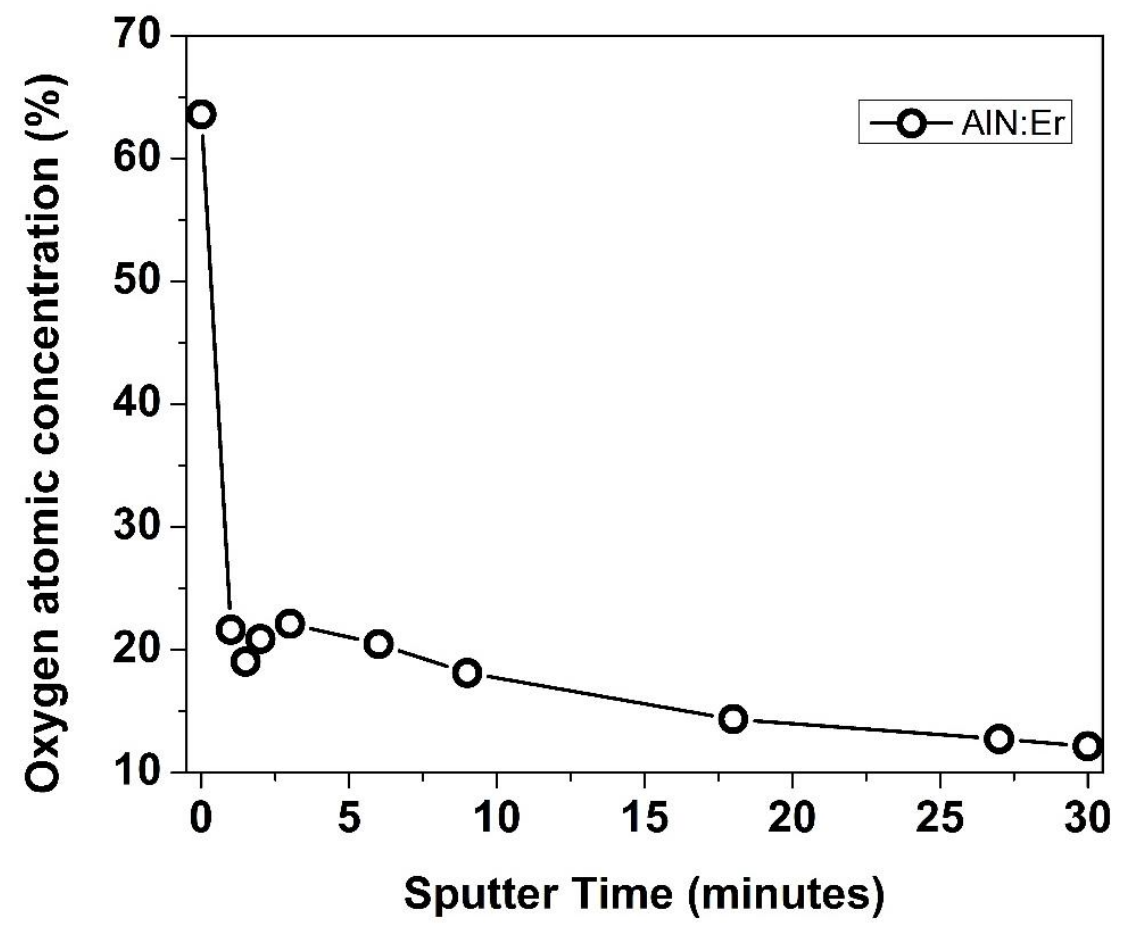

Figure 2.2: Oxygen atomic concentration in AlN:Er thin film as a function of Ar sputter time.

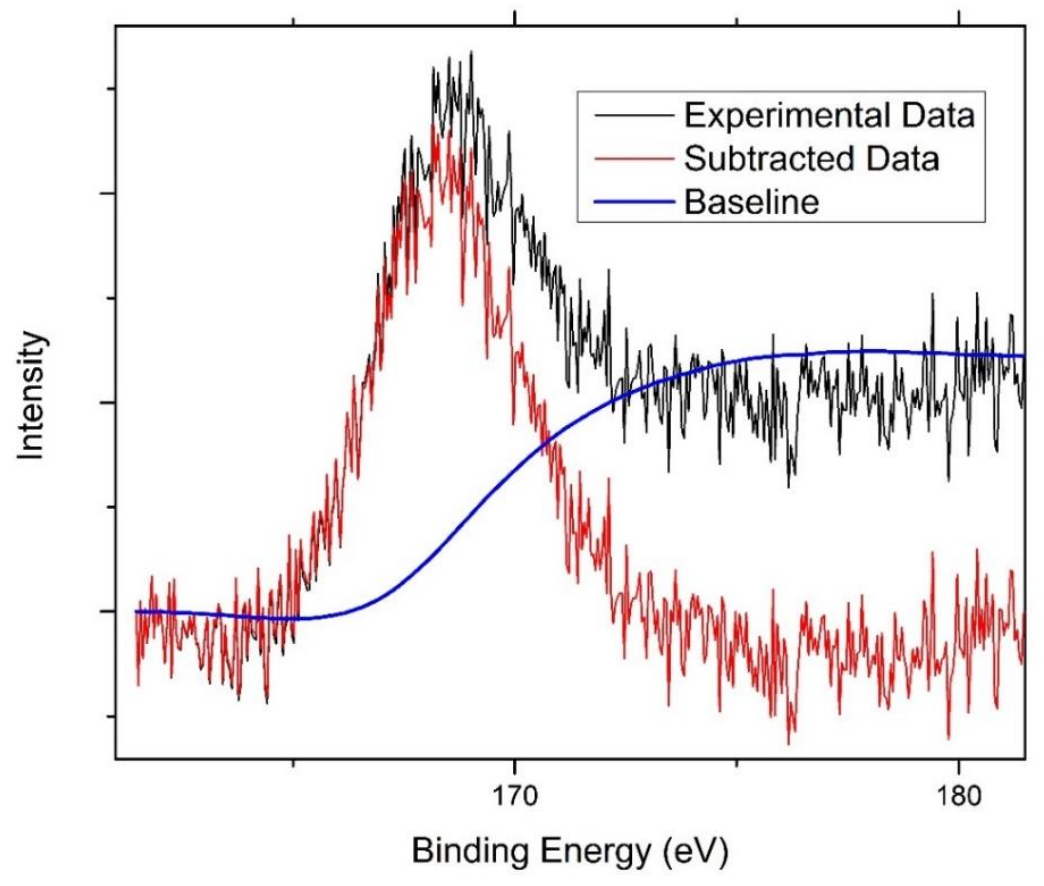

Figure 2.3: This figure shows Er (4d) peak before (black line), background (blue line) calculated by Shirley method and corrected peak after baseline subtraction (red line). 


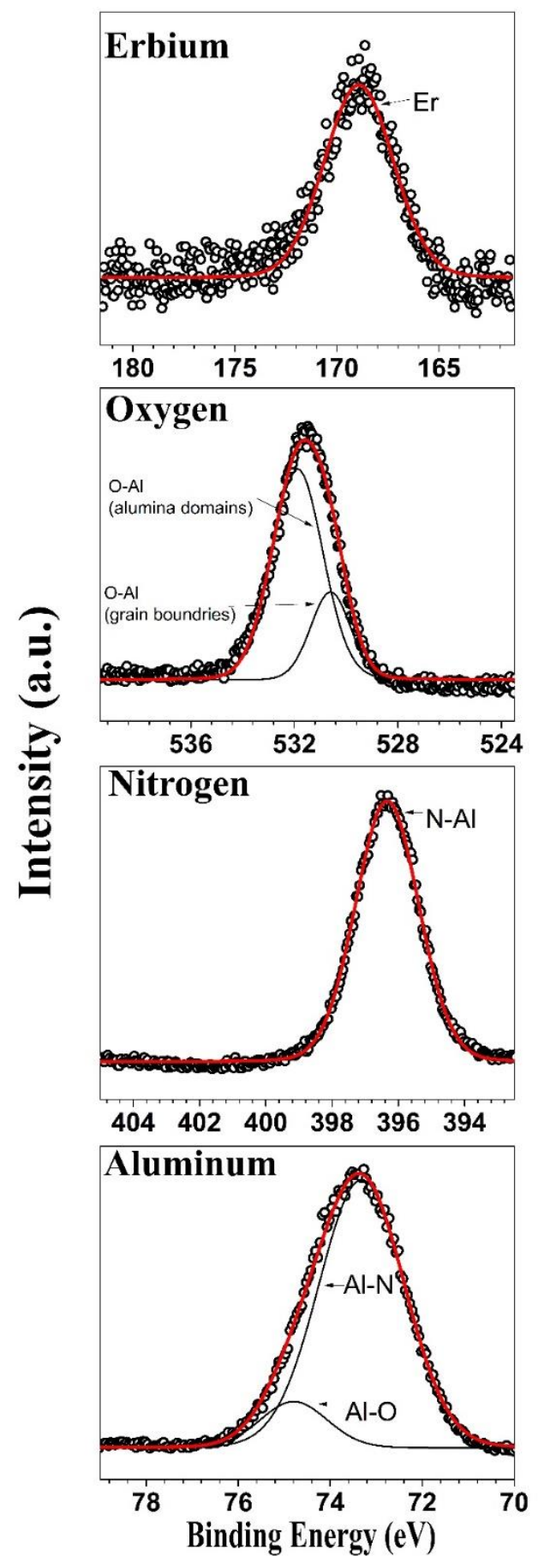

Figure 2.4: XPS plots of Al, N, O and Er peaks after 6 minutes of Argon ion sputtering of the AlN:Er thin film. Dots show the experimental data, black lines the peaks obtained after peak-fits for different chemical states and red line summation of the peaks for each element. 


\subsection{X-ray Diffraction (XRD):}

X-ray diffraction (XRD) measurements on the thin films were carried out using a Rigaku RU300 rotating anode system with $\mathrm{Cu}-\mathrm{K}_{\alpha}(\lambda=1.54185 \AA)$ source. A curved graphite crystal was used as the monochromator. These scans were performed from $2 \theta=20^{\circ}$ to $80^{\circ}$. The room temperature XRD patterns of the thin films of AlN, and AlN:Er are shown in Fig. 2.5. For the AlN film, the observed peaks can be assigned to either AlN or the Si- substrate. The observed peaks of AlN are (0002), (1011), $(10 \overline{1} 2),(11 \overline{2} 0)$ and $(10 \overline{1} 3)$ suggesting that the AlN film has no preferential orientation. The peak at $33^{\circ}$ is the second harmonic of the $\mathrm{Si}(004)$ peak, resulting due to the Bremsstrahlung effect. For the AlN:Er film, only the (0002) and the (0004) peaks are observed besides the Si peaks and the lines (10 $\overline{1} 1)$, $(10 \overline{1} 2),(11 \overline{2} 0)$ and $(10 \overline{1} 3)$ are absent suggesting preferential orientation of the film perpendicular to the c-axis after Er is introduced into the film. This preferential orientation of AlN:Er is considered to be an important reason for the several fold enhancement of the piezoelectric coefficient of AlN:Er thin films compared to Er free AlN films.

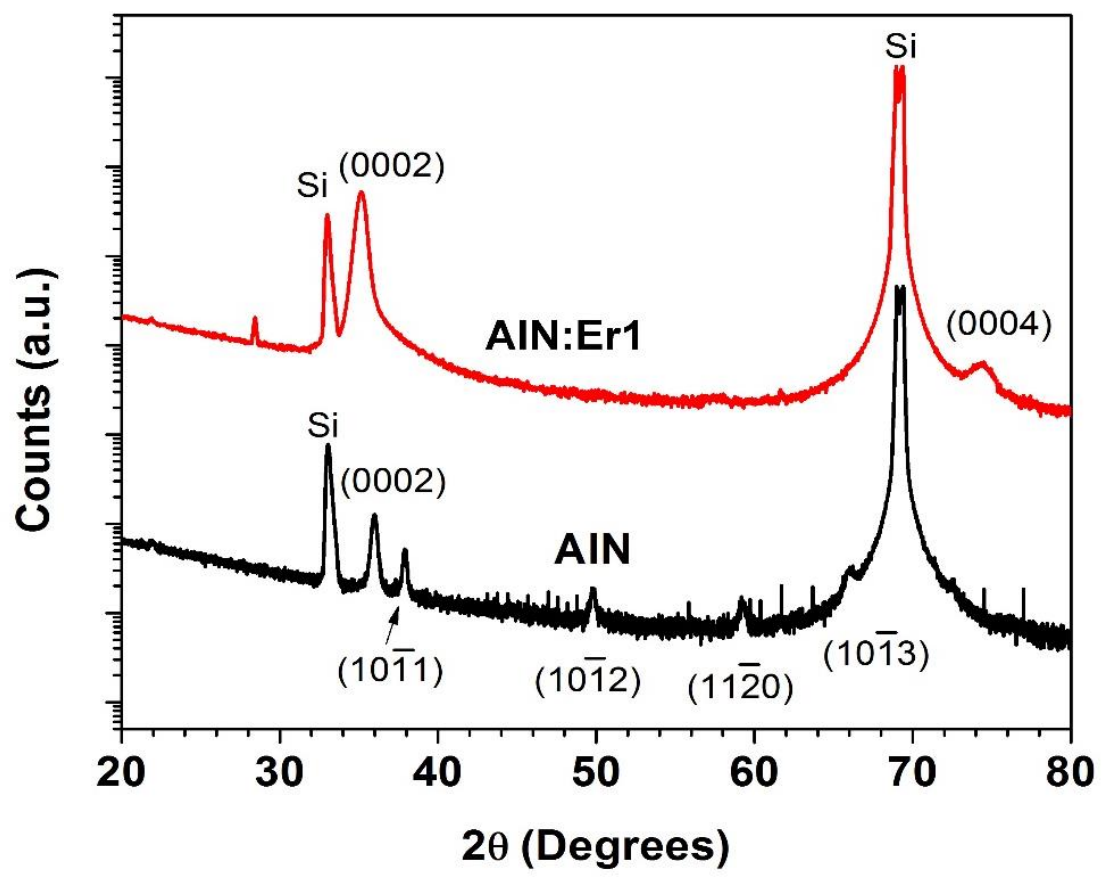

Figure 2.5: X-ray diffraction patterns for the AlN and AlN:Er1 (1\% Er concentration in the target) thin films on Si substrate.

XRD measurements on $\mathrm{Er}_{2} \mathrm{O}_{3}$ sample were performed using the Rigaku-DMax system with $\mathrm{Cu}$ $\mathrm{K}_{\alpha}(\lambda=1.5418 \AA)$ source from $2 \theta=5^{\circ}$ to $65^{\circ}$. The room temperature XRD patterns of the two samples of Erbium Oxide $\left(\mathrm{Er}_{2} \mathrm{O}_{3}\right)$, bulk powder sample of $\mathrm{b}-\mathrm{Er}_{2} \mathrm{O}_{3}$, and a nanocrystalline $\mathrm{Er}_{2} \mathrm{O}_{3}\left(\mathrm{n}-\mathrm{Er}_{2} \mathrm{O}_{3}\right)$ are 
shown in Fig. 2.6 along with the expected line positions for $\mathrm{Er}_{2} \mathrm{O}_{3}$ structure in the The International Centre for Diffraction Data (ICDD) data-base (\#00-008-0050). All the observed line match with expected line positions for $\mathrm{n}-\mathrm{Er}_{2} \mathrm{O}_{3}$ sample but for $\mathrm{b}-\mathrm{Er}_{2} \mathrm{O}_{3}$, there are two additional peaks (marked as 1 and 2 in Figure 2.6) which are identified as different forms of $\mathrm{SiO}_{2}$ and are present as impurities in the powder sample.

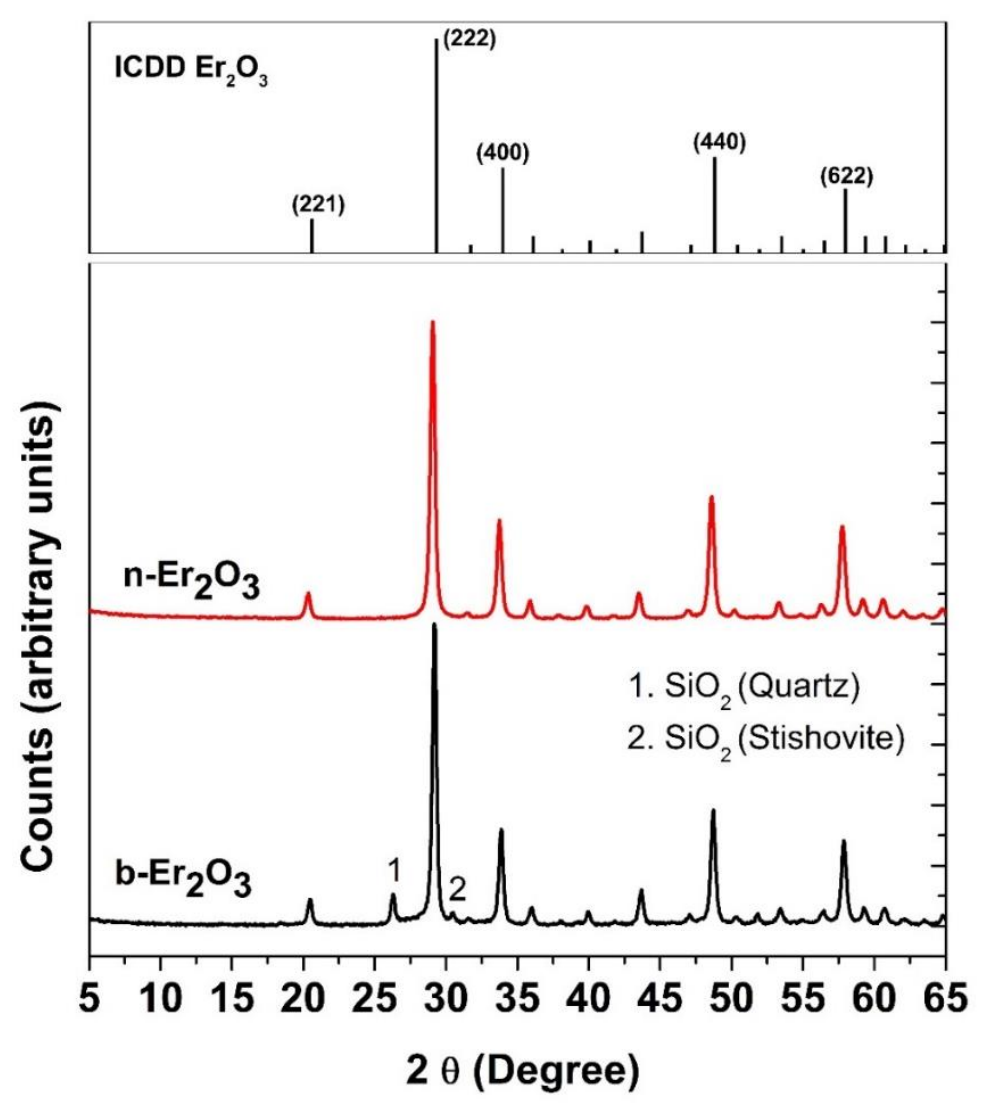

Figure 2.6: X-ray diffraction patterns of the two samples of $\mathrm{Er}_{2} \mathrm{O}_{3}$. For comparison, the expected line positions for $\mathrm{Er}_{2} \mathrm{O}_{3}$ along with Miller indices of a few prominent lines are shown at the top.

\subsection{Measurements of the Mass of Thin Films:}

Accurate magnitudes of sample masses are needed for analyzing the magnetic data presented in chapter 4 and 5. Whereas the samples of elemental Er and powder $\operatorname{Er}_{2} \mathrm{O}_{3}$ were weighed by an analytical balance, this procedure could not be used for finding the weights of the AlN and AlN:Er films deposited on Si substrate because of the weight of the substrate. Instead the lengths and the widths of the nearly rectangular films were measured with a Vernier scale, the thickness measured using edgeon scanning electron microscopy and variable angle spectroscopic ellipsometry, and density calculated from XRD measurements. For determining the thickness using SEM, the cross-section of the film was 
imaged. Figure 2.7 shows the cross-section for one of the AlN:Er thin film. For this film the calculated thickness was $1718 \pm 18 \mathrm{~nm}$. The length and width of the film were $8.58 \mathrm{~mm}$ and $2.97 \mathrm{~mm}$ respectively which gave the volume of the film equal to $4.378 \times 10^{-5} \mathrm{~cm}^{3}$. The elemental composition of the film determined using XPS was $\mathrm{Al}_{0.4225} \mathrm{Er}_{0.0108} \mathrm{~N}_{0.4125} \mathrm{O}_{0.1542}$ with molecular weight of $21.45 \mathrm{~g}$. The lattice parameters of the films determined using XRD measurements were: $c=5.11 \AA$ and $\mathrm{a}=3.19 \AA$. . This yields density of $3.122 \mathrm{~g} / \mathrm{cm}^{3}$ for the AlN:Er thin film. This calculated density, combined with the volume of the film gives $0.1367 \mathrm{mg}$ as mass of the thin film. The mass calculated this way is used to calculate the magnetic moment per unit mass of the thin film.

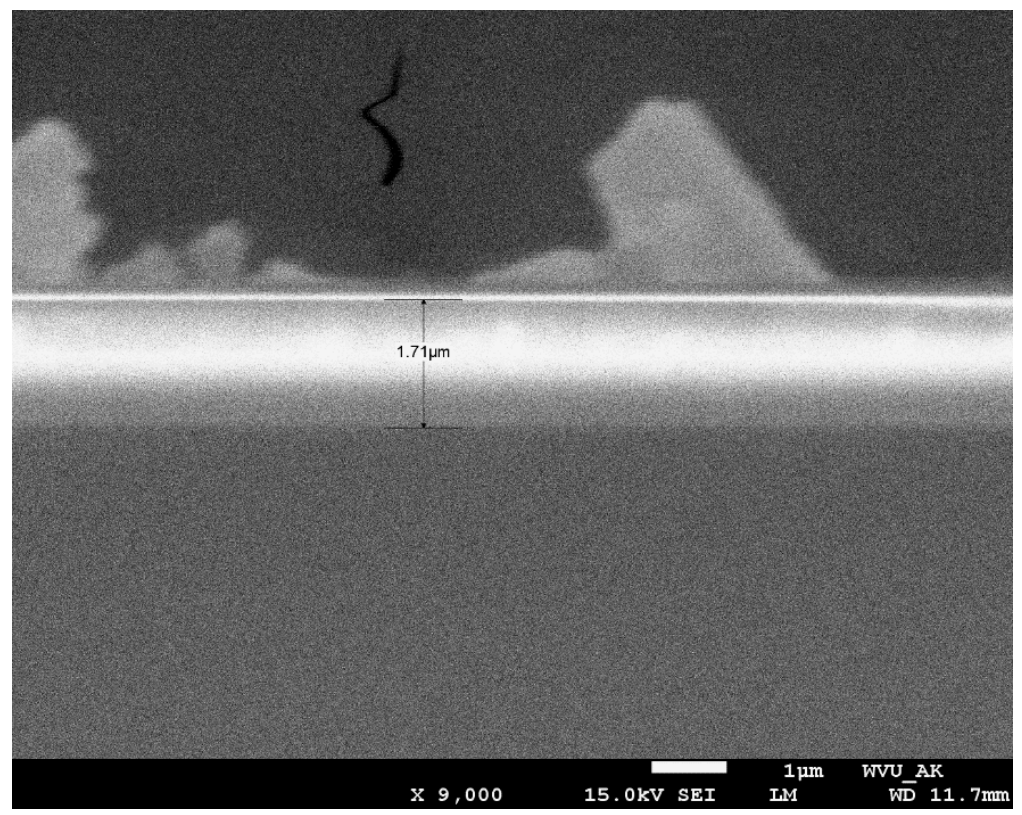

Figure 2.7: SEM image of cross-section of AlN:Er thin film.

\subsection{Measurements of Magnetization:}

Magnetic measurements on materials of interest here were performed using a commercial PPMS magnetometer from Quantum Design Inc. A magnetometer is based on principle of electromagnetic induction where changing magnetic flux in coil results into a generated potential difference across it. The basic design of a magnetometer consists of magnet, coils (called pickup coils) to measure the magnetization and a vibration unit to vibrate the sample. Fig. 2.8 shows a block diagram of a magnetometer. When a sample in magnetic field is made to vibrate near the pickup coils, it results in change in magnetic flux in the coils. According to Faraday's law of induction, the magnitude of potential across the coil is given by equation: 


$$
V=\frac{d \Phi}{d t}=-\left(\frac{d \Phi}{d z}\right)\left(\frac{d z}{d t}\right)
$$

where $\Phi$ is the total magnetic flux through the pickup coil and $\mathrm{z}$ is the vertical position of the sample with respect to the coil. The strength of this potential difference is used to measure the magnetic moment of the vibrating sample. Note that the magnetic moment per unit volume of the sample is magnetization by definition. The strength of the magnetic moment and the potential $\mathrm{V}$ depend on the motion of the vibrating sample. For a sample vibrating with frequency $f$ and amplitude $A$, it can be shown that magnetic moment $m$ of the sample is given by [32]:

$$
m=\frac{V}{2 \pi f C A \sin (2 \pi f t)}
$$

$\mathrm{C}$ being the coupling constant. To determine the coupling constant, the system is calibrated using a standard sample of Pd which has a magnetic susceptibility of $5.26 \times 10^{-6} \mathrm{emu} / \mathrm{gram}$ at room temperature, independent of magnetic field.

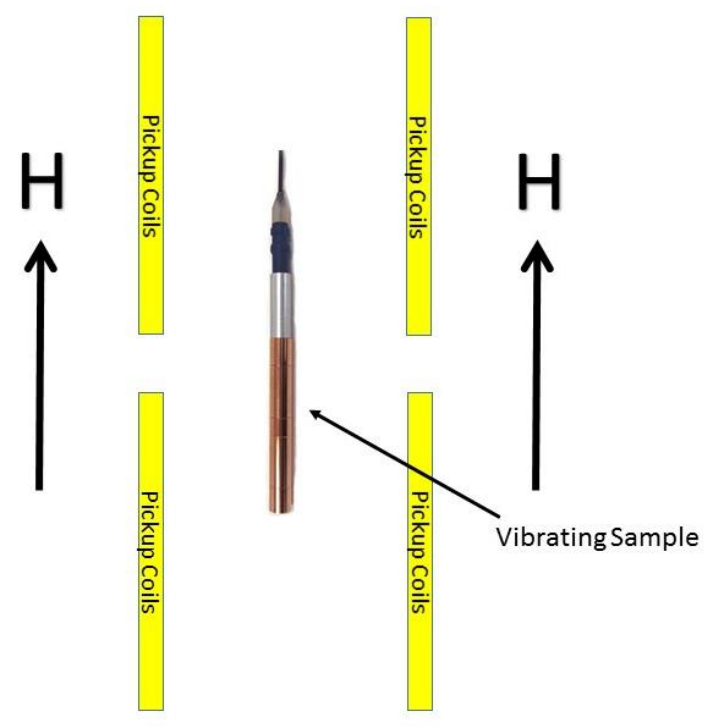

Figure 2.8: Schematic of a magnetometer.

The PPMS magnetometer used here has two different options: (i) Vibrating Sample Magnetometer (VSM) and (ii) AC/DC Magnetometry System (ACMS). The measurement on thin films (AlN and AlN:Er) were performed using VSM option of the PPMS, whereas the magnetic measurements on erbium metal and $\mathrm{Er}_{2} \mathrm{O}_{3}$ powder were carried out using the ACMS option of the 
PPMS. The difference between the two options is that where VSM can perform only DC magnetic measurements, PPMS is equipped with AC susceptometer along with DC magnetometer. Design of the VSM is such that it has a gradiometer coil and when the sample vibrates along with, it detects voltage induced in the coils by the magnetized sample synchronously. But in case of ACMS option, the sample moves through a number of coils and signal induced in each coil is measured and averaged to find the magnetic moment of the vibrating sample. In this work only DC magnetization measurements are performed, so the choice of VSM and ACMS option is purely based on the sample holder in the two options and the configuration and size of the samples investigated in this work. The design of the ACMS is such that AlN and AlN:Er thin film sample cannot be accommodated in ACMS option so VSM option was used for thin film samples.

\subsection{Measurements of Piezoelectric Coefficient:}

The measurements of the piezoelectric coefficient $\left(\mathrm{d}_{33}\right)$ measurement of AlN and AlN:Er thin films were performed using Polytec PSV-400 laser Doppler vibrometer (LDV). Measurement of the displacement in the piezoelectric thin films on application of electric field was carried out using the heterodyne interferometer [33]. In a laser based interferometer, when the laser beam falls on the object (in this case the $\mathrm{Pt} / \mathrm{AlN}: \mathrm{Er} / \mathrm{Si} / \mathrm{Pt}$ structure described in the previous section) subjected to an electric field, the frequency and the phase of the reflected light changes due to the vibrations of the sample. The changes in frequency and phase are determined to calculate the velocity and displacement of the object respectively. In Polytec PSV-400 system, the interferometer is modified Mach-Zehnder interferometer, the basic arrangement of which is shown in Fig. 2.9 [34].

In this system, using beam splitter BS1 in Fig. 2.9, the incident laser beam is divided into two parts, object beam and reference beam. This system is used to calculate the phase difference generated by the displacement of the target object and the reference beam. At the detector, the interference of the scattered beam from the object and reference beam takes place. The intensity of the resultant interference is given by the equation:

$$
I(\varphi)=\frac{I_{\max }}{2}(1+\cos \varphi)
$$

where $I_{\max }$ is the maximum resulting intensity (when the phase change $\varphi$ is zero). Phase change $\varphi$ is related to the path difference $\Delta \mathrm{L}$ by the equation:

$$
\varphi=2 \pi \cdot \frac{\Delta L}{\lambda}
$$

where $\lambda$ is the wavelength of laser. The resultant interference intensity I is converted into electrical signal and is used to determine the displacement of the target object. In this work the AC signal with 
frequency of $7.0 \mathrm{kHz}$ frequency and amplitude varying from a few volts to $40 \mathrm{~V}$ was applied to the sample to measure the $d_{33}$ coefficient. The results from these measurements are given in the following chapter 3.

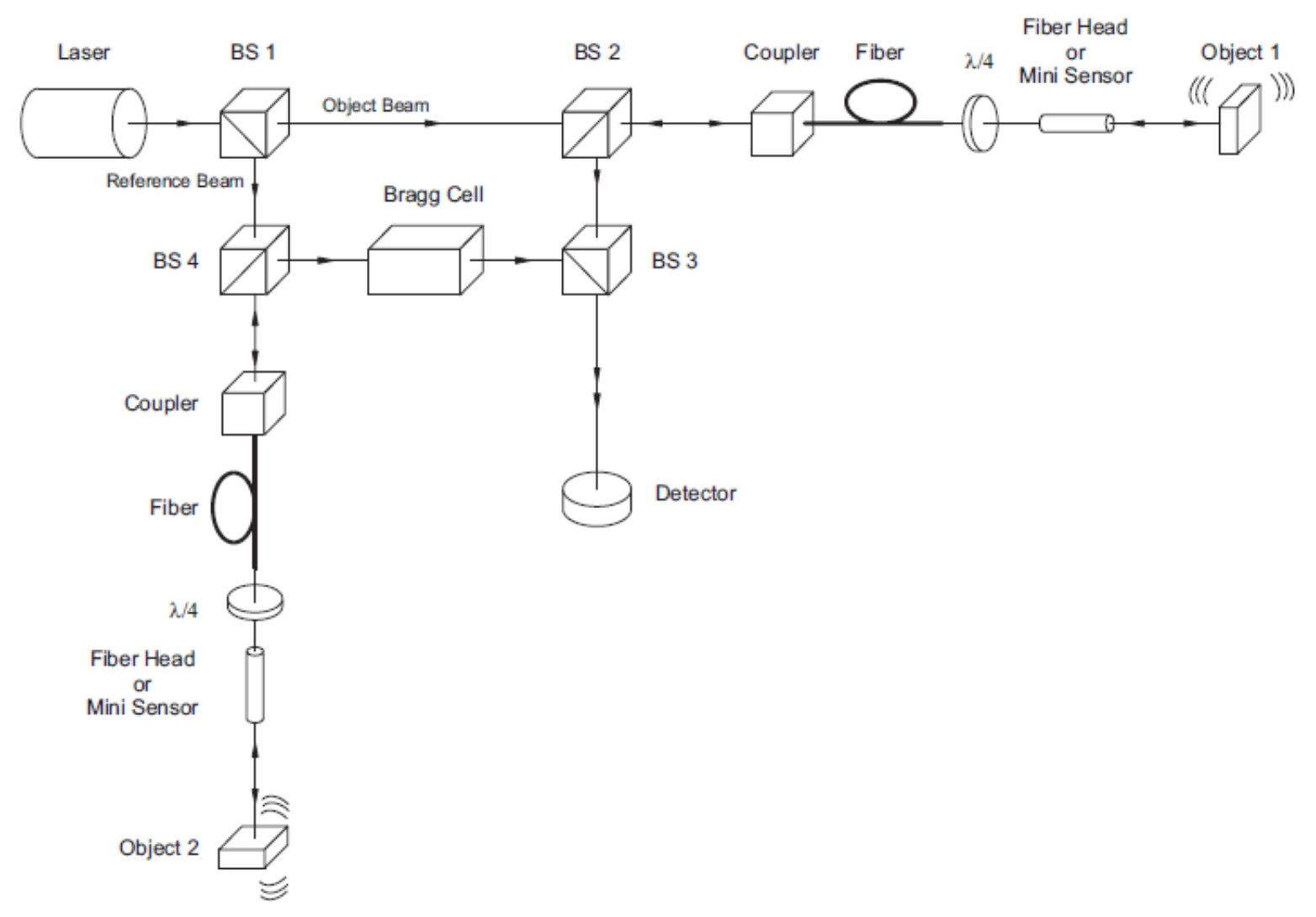

Figure 2.9: Schematic of optics in modified Mach-Zehnder interferometer in Polytec PSV-400 system (Taken from Ref. [34]).

\subsection{Platinum Contact Deposition on Thin Films for Piezoelectric Coefficient Measurements:}

For the measurements of the piezoelectric coefficient $\mathrm{d}_{33}$ (described in detail in Chapter 3) using Laser Doppler Vibrometer, platinum contacts were deposited using image reversal photolithography. The choice of platinum as top and bottom ( $p$ and $n$ ) contacts is based on the results reported by Harman et al. [35], where it is reported that piezoelectric coefficient $\mathrm{d}_{33}$ is not only function of the crystalline quality of the thin films, but also depends on the work function of the contact metals. Fig. 2.10 shows the piezoelectric coefficient on different AlN thin films as function of work function 
of the contact metals. As platinum with highest work function of $5.15 \mathrm{eV}$ shows highest $\mathrm{d}_{33}$ on AlN thin films, same is used here in this work on AlN and AlN:Er thin films.

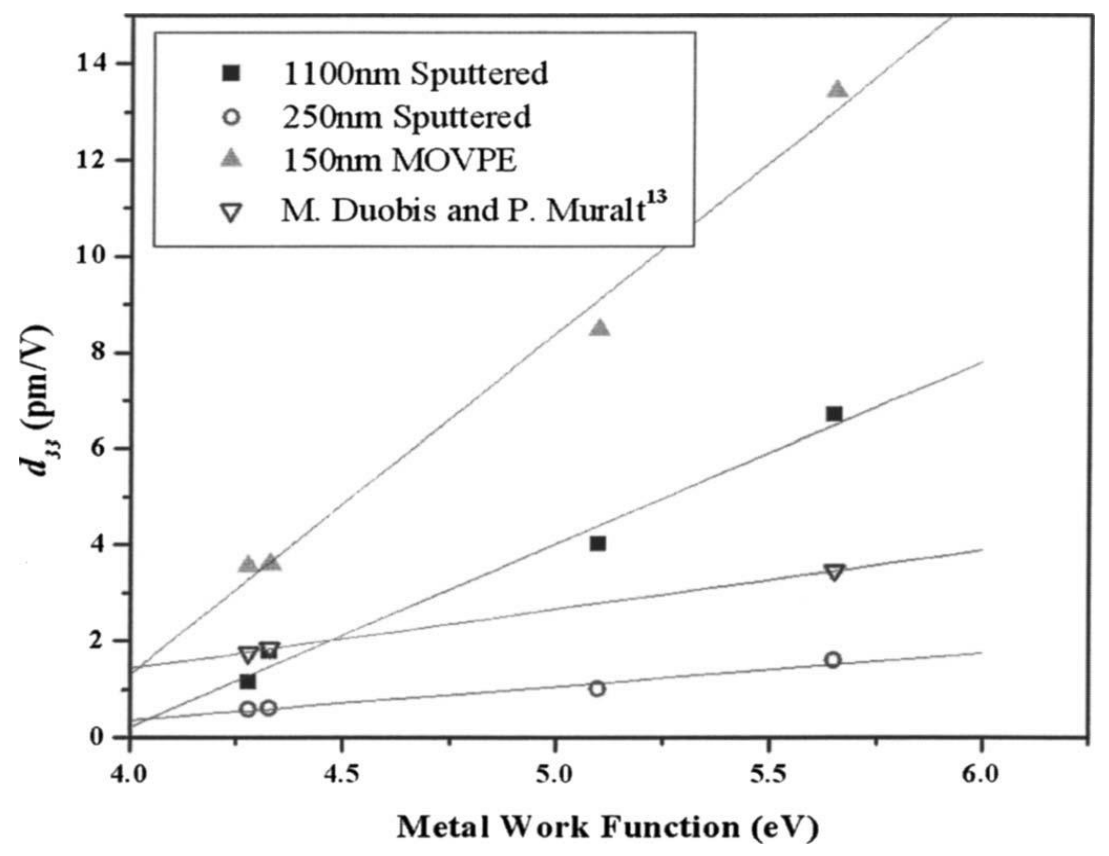

Figure 2.10: Piezoelectric coefficient $d_{33}$ for different AlN thin films as function of work functions of contact metals. The metals reported are $\mathrm{Al}, \mathrm{Ti}, \mathrm{Au}$ and $\mathrm{Pt}$ in order of increasing work function. (From Ref. [35]).

To deposit the top Pt contacts, image reversal photolithography was used. For this SUSS Microtech MAP Mask Aligner was used, which employs UV light (wavelength $320 \mathrm{~nm}$ ) exposure source. Photoresist used for photolithography is AZ 5214. The basic steps involved in photolithography are shown in Fig. 2.11. For this, first on clean thin film, we spin photoresist (4000 rpm, 40 seconds) to have an even, about $1.4 \mu \mathrm{m}$ thick layer of photoresist. To remove the solvent from the photoresist, it is followed by soft bake for 60 seconds at $90^{\circ} \mathrm{C}$. Using the mask aligner, then the sample is exposed to $50 \mathrm{~mJ}$ energy, followed by post exposure bake for 120 seconds at $120^{\circ} \mathrm{C}$. After the post exposure bake, using OAI UV Flood Exposure System (wavelength 365nm) sample is exposed to the $2000 \mathrm{~mJ}$ energy. After this, the sample is developed in MIF 300 developer solution for about 25 seconds. This stage is shown in step 2 of Fig. 2.11. The obtained pattern is verified using the alpha step and optical microscope. After removing any residual photoresist by dipping in water, the sample is dried with compressed nitrogen. After this, top Pt metal contacts (thickness $\sim 100 \mathrm{~nm}$ ) are deposited using DCMagnetron sputter station followed by back Pt metal contacts on Si substrate surface. The sample at this stage is shown in step 3 of Fig. 2.11. After this, photoresist is removed using the lift off process in acetone, giving the Pt contacts as shown in last step of Fig. 2.11. 


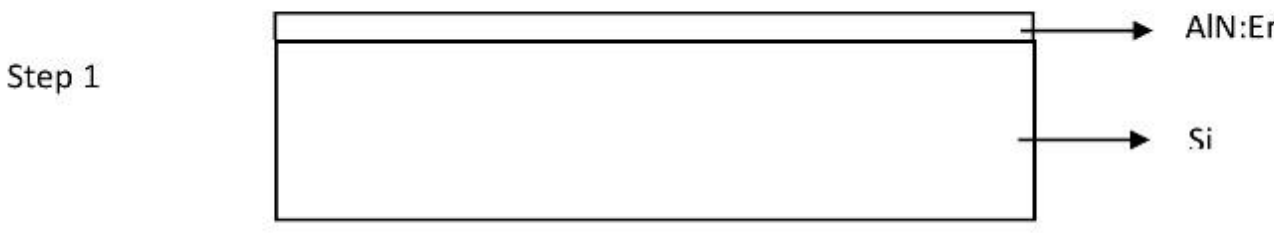

Step 2

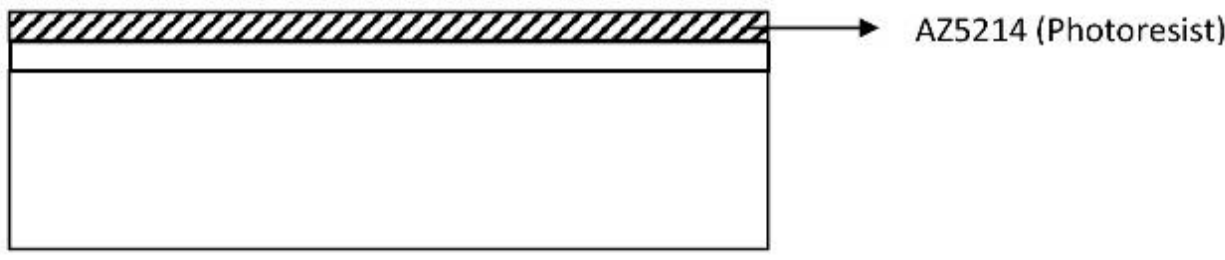

Step 3

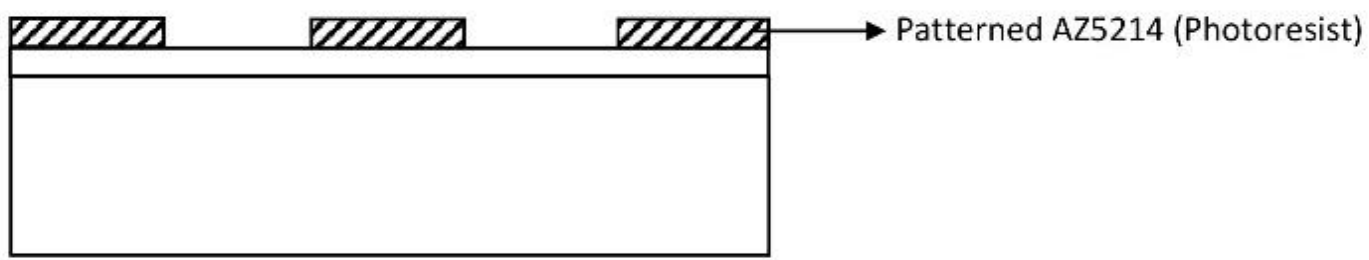

Step 4

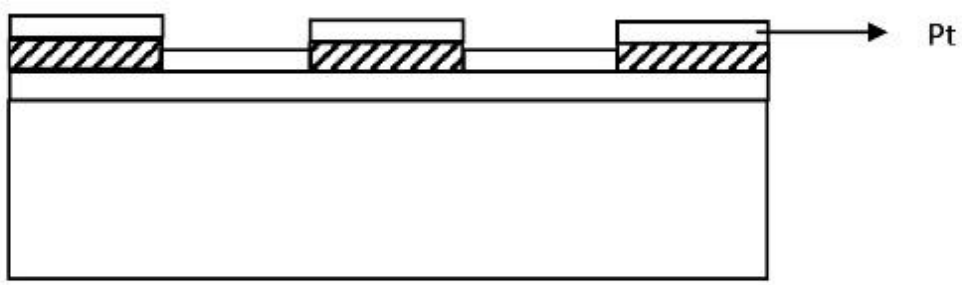

Step 5

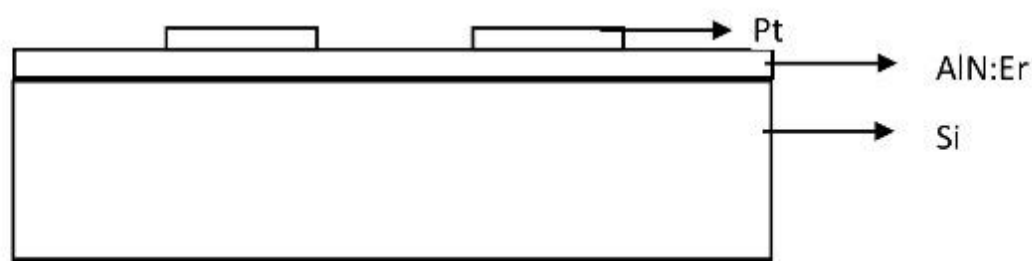

Figure 2.11: Main steps involved in image reversal photolithography to deposit Pt metal contacts on AlN and AlN:Er thin films for piezoelectric coefficient measurements. 


\section{CHAPTER 3 - Piezoelectric Properties of AIN:Er Thin Films}

\subsection{Introduction:}

In this chapter, the results of piezoelectric coefficient measurements on AlN and AlN:Er thin films are presented. In order to provide a proper theoretical basis for the discussion of the results, relationships between the piezoelectric properties of materials and their elastic and dielectric properties are first presented. After the piezoelectric properties, structural properties of the thin films are described based on the X-ray diffraction measurements. Results obtained from XRD measurements are used to explain the improved piezoelectric properties of AlN:Er thin films vis-à-vis Erbium free AlN thin films.

\subsection{Theoretical Considerations:}

When stress is applied to piezoelectric materials, it leads to the generation of electrical charge proportional to the applied stress. This phenomenon is defined as piezoelectric effect and the materials exhibiting this are called piezoelectric materials. Reverse of this effect, i.e. production of the strain on application of electric field is also shown by piezoelectric materials and this effect is known as reverse piezoelectric effect.

For the mathematical formulation of the piezoelectric effect in simplest form, the charge polarization $\mathbf{P}_{\mathrm{p}}$ produced in a material due to applied stress $\mathbf{T}$ is given by the equation [36],

$$
\boldsymbol{P}_{p}=d \cdot \boldsymbol{T}
$$

where $\mathrm{d}$ is the piezoelectric strain coefficient (All quantities in bold are vectors). Similarly for the piezoelectric materials, the ratio of the strain produced $\mathbf{S}_{\mathrm{p}}$ to the applied electric field $\mathbf{E}$ is also $\mathrm{d}$ and it is given by the equation,

$$
\boldsymbol{S}_{p}=d \cdot \boldsymbol{E}
$$

By Hooke's law, the strain is directly proportional to the applied stress, i.e. $\mathbf{T}=\mathrm{c} \cdot \mathbf{S}$ where $\mathrm{c}$ is the elastic constant for the material. Using this, Eqs. (3.1) and (3.2) can be modified as,

$$
\begin{aligned}
& \boldsymbol{P}_{p}=e \cdot \boldsymbol{S} \\
& \boldsymbol{T}_{\boldsymbol{p}}=e \cdot \boldsymbol{E}
\end{aligned}
$$

where $\mathrm{e}$ is the piezoelectric stress constant and subscript $\mathrm{p}$ in above quantities refer to the piezoelectric effect.

Next, when an electric field $\mathbf{E}$ is applied to a material of dielectric constant $\gamma$, the resulting polarization of the material causes surface charge density of magnitude $\mathbf{D}$ given by the equation, 


$$
\boldsymbol{D}=\gamma \cdot \boldsymbol{E}
$$

In the case of piezoelectric materials, the applied electric field will cause strain given by Eq. (3.2) and this strain produces polarization of the material given by $\mathbf{P}_{\mathrm{p}}=\mathrm{e} \cdot \mathbf{S}_{\mathrm{p}}$. The new surface charge density is then

$$
\boldsymbol{D}=\gamma \cdot \boldsymbol{E}+e \cdot \boldsymbol{S}_{p}
$$

The elastic stress produced by strain $\mathbf{S}_{\mathrm{p}}$ is given by the equation,

$$
\boldsymbol{T}_{e}=c \cdot \boldsymbol{S}_{p}
$$

Also the applied electric field will produce stress $\mathbf{T}_{\mathrm{p}}$ given by Eq. (3.4) and will be in opposite direction to the elastic stress given in Eq. (3.7). Stress $\mathbf{T}_{\mathrm{p}}$ produces the strain and elastic stress $\mathbf{T}_{\mathrm{e}}$ attempts to recover to the original structure. The net stress produced is given by

$$
\boldsymbol{T}_{\boldsymbol{e}}=c \cdot \boldsymbol{S}_{p}-e \cdot \boldsymbol{E}
$$

Once the two counteracting stresses balances each other in a material, it remains in strained and static state. On the other hand, if variable electric field is applied, the strain will vary and it will produce dynamic displacement in the material. Application of varying electric field to piezoelectric materials is commonly used for applications of these materials as transducers and actuators.

\subsection{Elastic Properties:}

Solids in general are anisotropic and so their physical properties are direction dependent. The framework outlined above is not sufficient to describe the elastic, dielectric and piezoelectric properties of materials. In the following section based on the text book by Kittel [37], these physical properties are described in tensor and matrix form and symmetry of the crystals is invoked to define the necessary independent constants to explain the elastic, piezoelectric and dielectric properties of the AlN and related materials.

The linear relationship between the generalized stress $\tau_{\mathrm{ij}}$ and generalized strain $\varepsilon_{\mathrm{kl}}$ is

$$
\tau_{i j}=\sum_{k l} c_{i j k l} \epsilon_{k l}
$$

where indices $\mathrm{i}, \mathrm{j}, \mathrm{k}, 1$ have values 1, 2, 3 and these values correspond to $\mathrm{x}, \mathrm{y}$ and $\mathrm{z}$-axes respectively. As shown in figure 3.1, there are 9 different $\tau_{\mathrm{ij}}$ and similarly 9 different $\varepsilon_{\mathrm{kl}}$. So the elastic stiffness

constants $c_{i j k l}$ have 81 different values. By considering the static equilibrium in the cube shown in figure 3.1, it can be inferred that no rotation is taking place or in other words stresses $\tau_{i j}=\tau_{j i}$, and strains $\epsilon_{k l}=\epsilon_{l k}$ which reduces the independent values of both $\tau_{k l}$ and $\epsilon_{k l}$ to 6 and hence the total 
number of stiffness constants $c_{i j k l}$ to 36 . As interchangeability of $\mathrm{i}$ and $\mathrm{j}$ suffix in $\tau_{i j}$ and suffixes $\mathrm{k}$ and 1 in $\varepsilon_{\mathrm{kl}}$ makes it unnecessary to use four suffixes for stiffness constants and they are replaced using the following notation:

$\begin{array}{rrrrrr}11 & 22 & 33 & 23,32 & 31,13 & 12,21 \\ \downarrow & \downarrow & \downarrow & \downarrow & \downarrow & \downarrow \\ 1 & 2 & 3 & 4 & 5 & 6\end{array}$

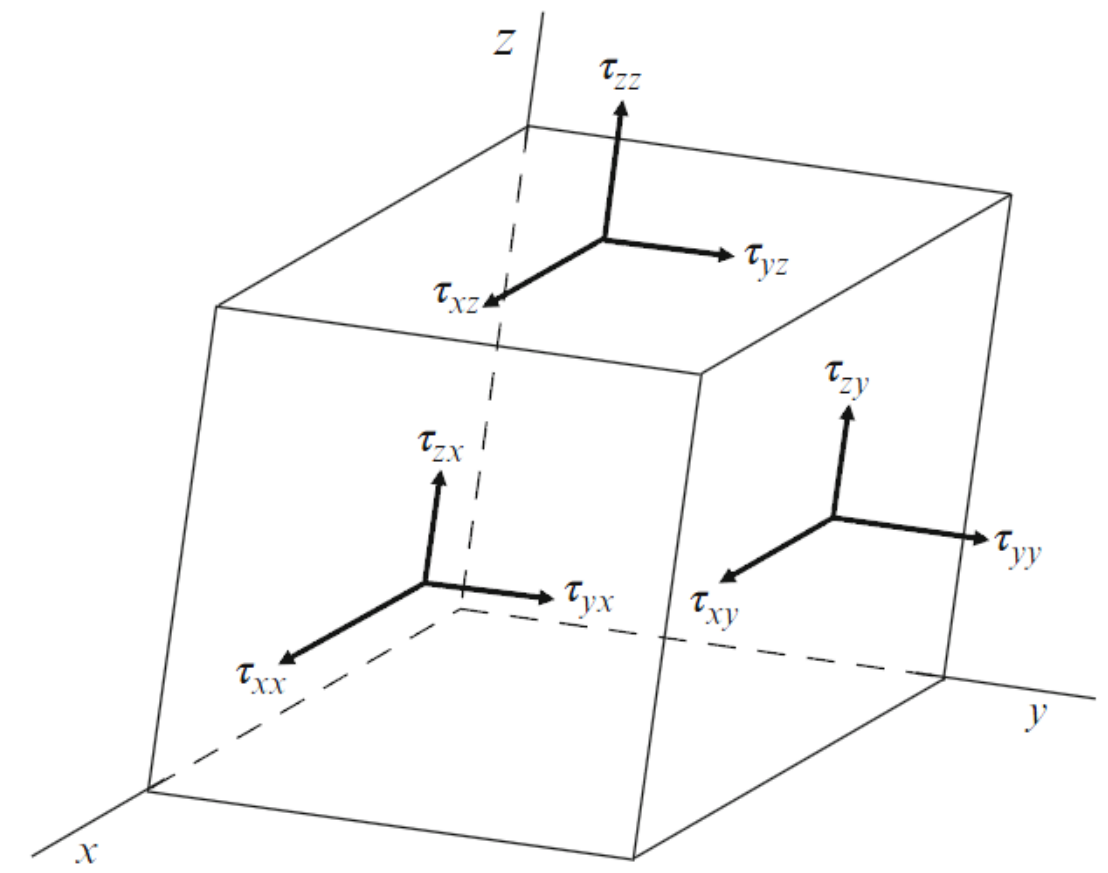

Figure 3.1: Stress components on the surface of an infinitesimal cube (Taken from Ref. [38]).

Therefore the relation given in Eq.(3.9) in matrix form can be written as:

$$
\left[\begin{array}{l}
\tau_{x x} \\
\tau_{y y} \\
\tau_{z z} \\
\tau_{y z} \\
\tau_{z x} \\
\tau_{x y}
\end{array}\right]=\left[\begin{array}{llllll}
c_{11} & c_{12} & c_{13} & c_{14} & c_{15} & c_{16} \\
c_{21} & c_{22} & c_{23} & c_{24} & c_{24} & c_{24} \\
c_{31} & c_{32} & c_{33} & c_{34} & c_{35} & c_{36} \\
c_{41} & c_{42} & c_{43} & c_{44} & c_{45} & c_{46} \\
c_{51} & c_{52} & c_{53} & c_{54} & c_{55} & c_{56} \\
c_{66} & c_{62} & c_{63} & c_{64} & c_{65} & c_{66}
\end{array}\right]\left[\begin{array}{c}
\epsilon_{x x} \\
\epsilon_{y y} \\
\epsilon_{z z} \\
\epsilon_{y z} \\
\epsilon_{z x} \\
\epsilon_{x y}
\end{array}\right]
$$

As discussed in Introduction to Solid State Physics by Kittel [37], elastic energy per unit volume (U) is the quadratic function of the strains and equation of $U$ can be written as: 


$$
U=\frac{1}{2} \sum_{i=1}^{6} \sum_{j=1}^{6} c_{i j} \epsilon_{i} \epsilon_{j}
$$

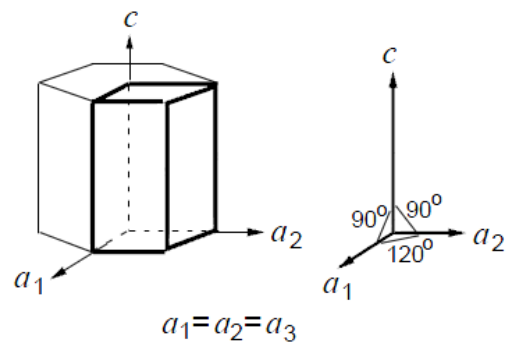

(a)

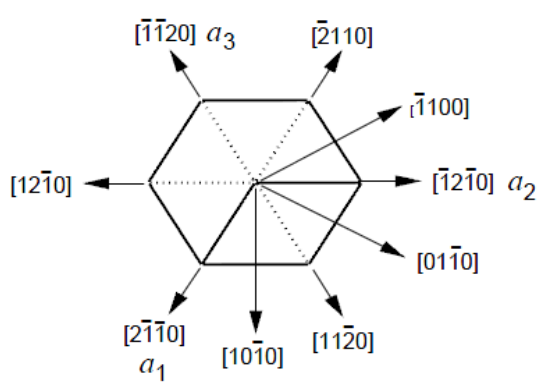

(b)

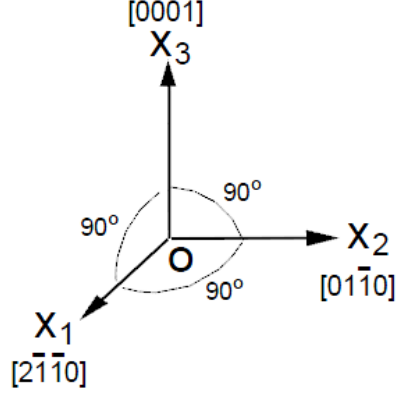

(c)

Figure 3.2: (a) Unit cell showing the $\mathrm{a}_{1}, \mathrm{a}_{2}$, and c-axes. (b) Directions in Miller-Bravais indices. (c) Relationship between $\mathrm{x}_{1}, \mathrm{x}_{2}$ and $\mathrm{x}_{3}$ axes in Fig. 3.1 and crystallographic directions (Taken from Ref. [1]).

where as stated in Eq. (3.10), $1 \equiv \mathrm{xx} ; 2 \equiv \mathrm{yy} ; 3 \equiv \mathrm{zz} ; 4 \equiv \mathrm{yz} ; 5 \equiv \mathrm{zx}$ and $6 \equiv \mathrm{xy}$. Stresses are related to the elastic energy density by the Eq.:

$$
\tau_{i j}=\frac{\partial U}{\partial e_{i j}}
$$

Using this relation it can be shown that in stress-strain relations, only the combinations of $\frac{1}{2}\left(c_{i j}+c_{j i}\right)$ enter in the equation, indicating that elastic stiffness constants are symmetric i.e. $c_{i j}=c_{j i}$ for $\mathrm{i} \neq \mathrm{j}$, which reduces the number of independent stiffness constants from 36 to 21.

Depending upon the symmetry of the crystal structure of the material, the number of independent elastic stiffness constants are reduced further. AlN has hexagonal wurtzite crystal structure with $6 \mathrm{~mm}$ symmetry. Unit cell of hexagonal structure is shown in Figure 3.2 (a), with two axes $\mathrm{a}_{1}$ and $a_{2}$ in one plane with angle of $120^{\circ}$ with each other and third axis $a_{3}$ perpendicular to this plane. Commonly planes in wurtzite structure are expressed in four miller indices scheme with axes $a_{1}, a_{2}, a_{3}$ in a plane and axes c orthogonal to the other three axes. This set of axes is shown in Fig. 3.3(b). Relationship expressed in Eq. (3.13) corresponds to three orthogonal axes and for this purpose, directions [2 $\overline{1} \overline{1} 0],[01 \overline{1} 0],[0001]$ are selected as $\mathrm{x}, \mathrm{y}$ and z-axis and are shown in Fig. 3.3 (c). As 
shown in [39], wurtzite structure which has $6 \mathrm{~mm}$ symmetry with six-fold rotational symmetry with respect to z-axis; with this symmetry, the elastic energy density U expressed in Eq. (3.12) changes to:

$$
U=\frac{c_{11}}{2}\left(\epsilon_{x x}^{2}+\epsilon_{y y}^{2}\right)+\frac{c_{33}}{2} \epsilon_{z z}^{2}+c_{12} \epsilon_{x x} \epsilon_{y y}+c_{13}\left(\epsilon_{x x}+\epsilon_{y y}\right) \epsilon_{z z}+2 c_{44}\left(\epsilon_{y z}^{2}+\epsilon_{z x}^{2}\right)+2 c_{66} \epsilon_{x y}^{2}
$$

Then, using Eqs. (3.13) and (3.14),

$$
\begin{aligned}
& \tau_{x x}=c_{11} \epsilon_{x x}+c_{12} \epsilon_{y y}+c_{13} \epsilon_{z z} \\
& \tau_{y y}=c_{11} \epsilon_{y y}+c_{12} \epsilon_{x x}+c_{13} \epsilon_{z z}
\end{aligned}
$$

Comparing these two equations with Eq. (3.11) gives $c_{12}=c_{21} ; c_{14}=c_{15}=c_{16}=c_{24}=c_{25}=$ $c_{26}=0$. On carrying out this process further for other stresses, Eq. (3.13) reduces to Eq. (3.17) with $c_{66}=\frac{c_{12}-c_{12}}{2}$ with five independent elastic stiffness components.

$$
\left[\begin{array}{l}
\tau_{x x} \\
\tau_{y y} \\
\tau_{z z} \\
\tau_{y z} \\
\tau_{z x} \\
\tau_{x y}
\end{array}\right]=\left[\begin{array}{cccccc}
c_{11} & c_{12} & c_{13} & 0 & 0 & 0 \\
c_{12} & c_{11} & c_{13} & 0 & 0 & 0 \\
c_{13} & c_{13} & c_{33} & 0 & 0 & 0 \\
0 & 0 & 0 & c_{44} & 0 & 0 \\
0 & 0 & 0 & 0 & c_{44} & 0 \\
0 & 0 & 0 & 0 & 0 & c_{66}
\end{array}\right]\left[\begin{array}{c}
\epsilon_{x x} \\
\epsilon_{y y} \\
\epsilon_{z z} \\
\epsilon_{y z} \\
\epsilon_{z x} \\
\epsilon_{x y}
\end{array}\right]
$$

Similar to Eq.(3.9), relation between strain components, stresses and elastic compliance coefficients can be written as:

$$
\epsilon_{i j}=\sum_{k l} s_{i j k l} \tau_{k l}
$$

which on simplification reduces to:

$$
\left[\begin{array}{l}
\epsilon_{x x} \\
\epsilon_{y y} \\
\epsilon_{z z} \\
\epsilon_{y z} \\
\epsilon_{z x} \\
\epsilon_{x y}
\end{array}\right]=\left[\begin{array}{cccccc}
s_{11} & s_{12} & s_{13} & 0 & 0 & 0 \\
s_{12} & s_{11} & s_{13} & 0 & 0 & 0 \\
s_{13} & s_{13} & s_{33} & 0 & 0 & 0 \\
0 & 0 & 0 & s_{44} & 0 & 0 \\
0 & 0 & 0 & 0 & s_{44} & 0 \\
0 & 0 & 0 & 0 & 0 & s_{66}
\end{array}\right]\left[\begin{array}{c}
\tau_{x x} \\
\tau_{y y} \\
\tau_{z z} \\
\tau_{y z} \\
\tau_{z x} \\
\tau_{x y}
\end{array}\right]
$$

The stiffness compliance coefficients $s_{i j}$ are related to elastic coefficients $c_{i j}$ by equation:

$$
S_{i j}=\frac{(-1)^{i+j} \Delta_{i j}^{c}}{\Delta^{c}}
$$

where $\Delta^{c}$ is determinant of the corresponding matrix and $\Delta_{i j}^{c}$ is the adjunct of the term ij in the matrix. 


\subsection{Dielectric Properties:}

For anisotropic solids, the dielectric constant $\gamma$ given in Eq.(3.5) is also a tensor whose components in general are related to the applied electric field components and electric charge displacement by the following equation:

$$
\left[\begin{array}{l}
D_{1} \\
D_{2} \\
D_{3}
\end{array}\right]=\left[\begin{array}{lll}
\gamma_{11} & \gamma_{12} & \gamma_{13} \\
\gamma_{21} & \gamma_{22} & \gamma_{23} \\
\gamma_{31} & \gamma_{32} & \gamma_{33}
\end{array}\right]\left[\begin{array}{l}
E_{1} \\
E_{2} \\
E_{3}
\end{array}\right]
$$

where subscripts 1,2 and 3 corresponds to $\mathrm{X}, \mathrm{Y}$ and $\mathrm{Z}$ directions respectively.

Similarly, component $\mathrm{P}_{\mathrm{i}}$ of the polarization are related to susceptibility tensor $\chi_{\mathrm{ij}}$ by the equation:

$$
\left[\begin{array}{l}
P_{1} \\
P_{2} \\
P_{3}
\end{array}\right]=\left[\begin{array}{lll}
\chi_{11} & \chi_{12} & \chi_{13} \\
\chi_{21} & \chi_{22} & \chi_{23} \\
\chi_{31} & \chi_{32} & \chi_{33}
\end{array}\right]\left[\begin{array}{l}
E_{1} \\
E_{2} \\
E_{3}
\end{array}\right]
$$

Transverse components for both permittivity and susceptibility are non-zero only in case of triclinic and monoclinic crystal structures. For wurtzite crystal structure, there are only two independent components for both $\varepsilon_{\mathrm{ij}}$ and $\chi_{\mathrm{ij}}$, which are $\varepsilon_{11}=\varepsilon_{22}$ and $\varepsilon_{33}$ and similarly $\chi_{11}=\chi_{22}$ and $\chi_{33}$. All other components are equal to zero.

\subsection{Piezoelectric Properties:}

As described above, there are six independent components of stress and strain and three directions for polarization. Therefore Eqs. (3.1) and (3.3) can be written in tensor form as [40]:

$$
\begin{aligned}
& P_{i}=e_{i j k} \tau_{j k} \\
& P_{i}=d_{i j k} \epsilon_{j k}
\end{aligned}
$$

with $\mathrm{i}, \mathrm{j}, \mathrm{k}=1,2,3$. Here $e_{i j k}$ and $d_{i j k}$ are respectively stress and strain piezoelectric coefficients. Equations (3.23) and (3.24) represent the direct piezoelectric effect. Reverse piezoelectric effect was theoretically predicted by Lippmann [41] who applied thermodynamic potentials to come to the conclusion that if an object is subjected to electric field $\mathrm{E}$, under stress $\tau$, which results into polarization $\mathrm{P}$ and strain $\varepsilon$, and if the process is reversible, then their relation can be written as:

$$
\left(\frac{\partial P}{\partial \tau}\right)_{E}=\left(\frac{\partial \epsilon}{\partial E}\right)_{T}=d
$$

Here $d$ is the piezoelectric strain coefficient and Eq.(3.25) states that ratio of polarization generated on application of stress $\tau$ (direct piezoelectric effect) is equal to the ratio of strain produced $\varepsilon$ and the 
applied electric field E. Combining the elastic, dielectric and piezoelectric properties, the total strain in a piezoelectric material and electric charge displacement is given by the equations:

$$
\begin{gathered}
\epsilon_{i}=s_{i j} \tau_{j}+d_{m i} E_{m} \\
D_{m}=d_{m j} \tau_{j}+\epsilon_{m n} E_{n}
\end{gathered}
$$

where $\mathrm{i}, \mathrm{j}=1$ to 6 and $\mathrm{m}, \mathrm{n}=1$ to 3. Equation (3.27) represents reverse piezoelectric effect and Eq.(3.28) represents direct piezoelectric effect. Stress and strain piezoelectric coefficients $d_{m i}$ and $e_{m i}$ are related to each other by equations:

$$
\begin{aligned}
& e_{m i}=d_{m j} c_{j i} \\
& d_{m i}=e_{m j} s_{i j}
\end{aligned}
$$

For the wurtzite structure, the stress piezoelectric coefficient has only three non-zero independent components $[42,43]$ and in matrix form the equation can be written as:

$$
\left[\begin{array}{l}
S_{1} \\
S_{2} \\
S_{3} \\
S_{4} \\
S_{5} \\
S_{6}
\end{array}\right]=\left[\begin{array}{cccccc}
0 & 0 & 0 & 0 & d_{15} & 0 \\
0 & 0 & 0 & d_{15} & 0 & 0 \\
d_{31} & d_{31} & d_{33} & 0 & 0 & 0
\end{array}\right]\left[\begin{array}{l}
E_{1} \\
E_{2} \\
E_{3}
\end{array}\right]
$$

In this work, electric field is applied only in c-axis direction i.e. $\mathrm{E}_{1}=\mathrm{E}_{2}=0$. Therefore, the non-zero strain produced are $S_{1}, S_{2}$ and $S_{3}$, which are all longitudinal. Strain $S_{3}$ is measured experimentally, which provides the piezoelectric coefficient $\mathrm{d}_{33}$. Measurements of $\mathrm{d}_{33}$ are reported in this work.

\subsection{Experimental Results on Piezoelectric Coefficient of AIN:Er Thin Films:}

For the measurements of the piezoelectric coefficient $\left(\mathrm{d}_{33}\right)$ of AlN:Er thin films, the process of thin film deposition is described in Chapter 2. The thin films for this work were deposited at sputtering target power of $200 \mathrm{~W}$. However, to reach this decision, thin films were deposited using the sputter power of 100,150,170,200, 220 and $250 \mathrm{~W}$ followed by measuring the refractive index of the films using Variable Angle Spectroscopic Ellipsometry (VASE) since it is known that refractive index of a thin film is the measure of its structure order. Results for AlN:Er (3\% Er) thin films of $450 \mathrm{~nm}$ are shown in Fig. 3.3. It is observed that films deposited at $170 \mathrm{~W}$ and $200 \mathrm{~W}$ power have nearly the same refractive index. Although the film deposited at $240 \mathrm{~W}$ has slightly higher refractive index, the target was found to get heated at this power. Therefore the power of $200 \mathrm{~W}$ was considered to be the optimum for depositing the films. 
It is noted that amorphous thin films of AlN have refractive indices in the range of 1.8-1.9, polycrystalline thin films have refractive indices in range of 1.9-2.1 and epitaxial grown films have refractive index is in the range of 2.1 to 2.2 [44]. Hence refractive index is used here as a metric to decide on the use of appropriate deposition target power. The measured refractive index of AlN:Er thin film shown in Fig.3.3 is close to 2.11 signifying poly-crystallinity with high structural order.

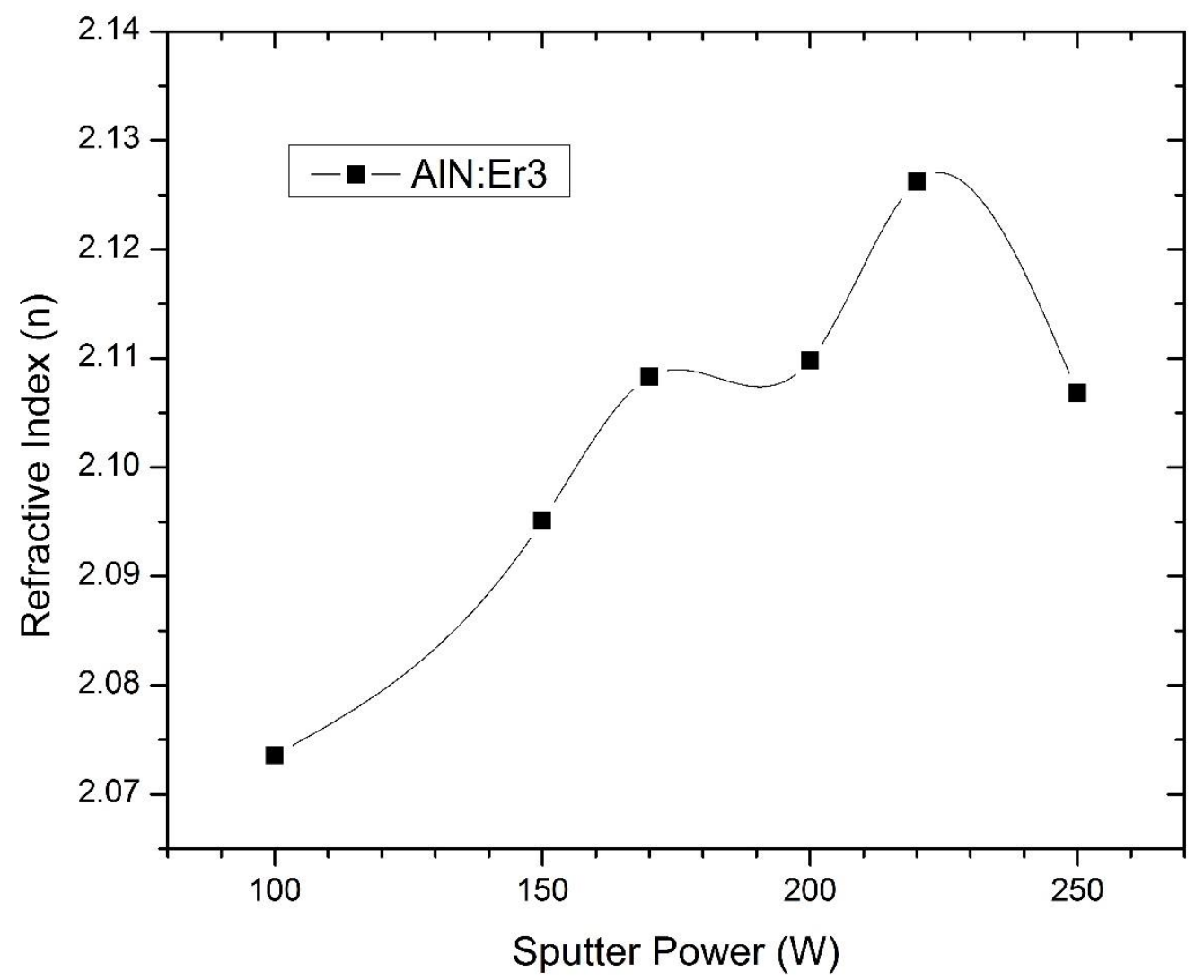

Figure 3.3: Refractive index of AlN:Er thin film as function of sputtering target power. The line connecting the data points is visual guide.

Laser Doppler Vibrometer (LDV) describe in Chapter 2 was used for the measurements of the $\mathrm{d}_{33}$ coefficient measurement. In this technique, ac signal is applied between the top and bottom contacts of the thin film with voltage ranging from a few volts to $40 \mathrm{~V}$. To decide on the use of a particular frequency of the ac signal for the measurements, criterion was used where displacement and hence $\mathrm{d}_{33}$ is relatively independent of ac frequency. For this purpose, displacement of the thin film was measured at frequency value ranging from $2 \mathrm{kHz}$ to $18 \mathrm{kHz}$. As shown in Fig. 3.4, the displacement is relatively independent of frequency in range of $5 \mathrm{kHz}$ to $9 \mathrm{kHz}$. For this reason, frequency of $7 \mathrm{kHz}$ was selected for further $\mathrm{d}_{33}$ coefficient measurement. The plot of displacement as function of applied voltage in Fig. 
3.5 shows nearly linear behavior. For a displacement $\delta$ and applied voltage $V$, the coefficient $\mathrm{d}_{33}$ is then given by the equation:

$$
d_{33}=\frac{\delta}{V}
$$

Since the displacement varies linearly with the applied voltage in Fig. 3.5, the slope of this plot gives the piezoelectric coefficient for the given measurement.

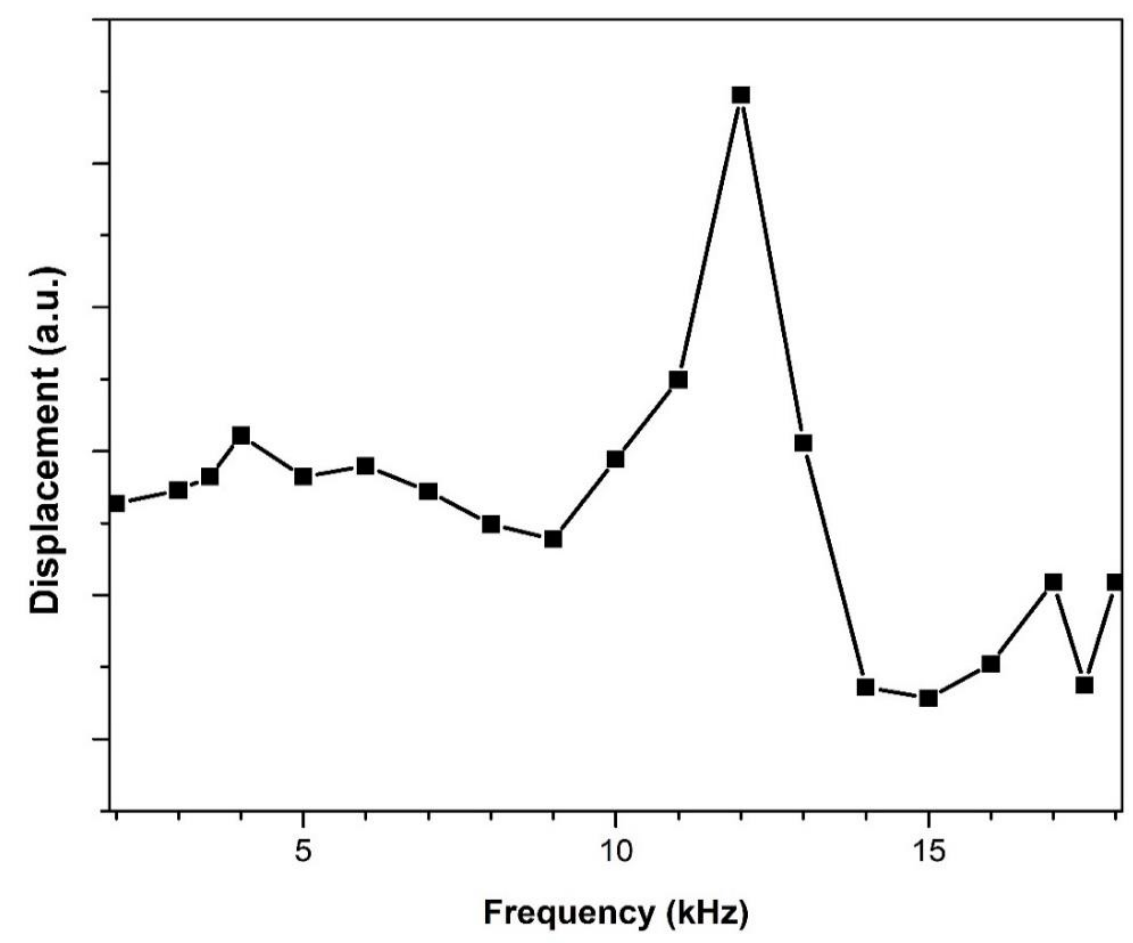

Figure 3.4: AlN:Er displacement as function of ac frequency at V $=40 \mathrm{~V}$. The lines connecting the data points are for visual guide. 


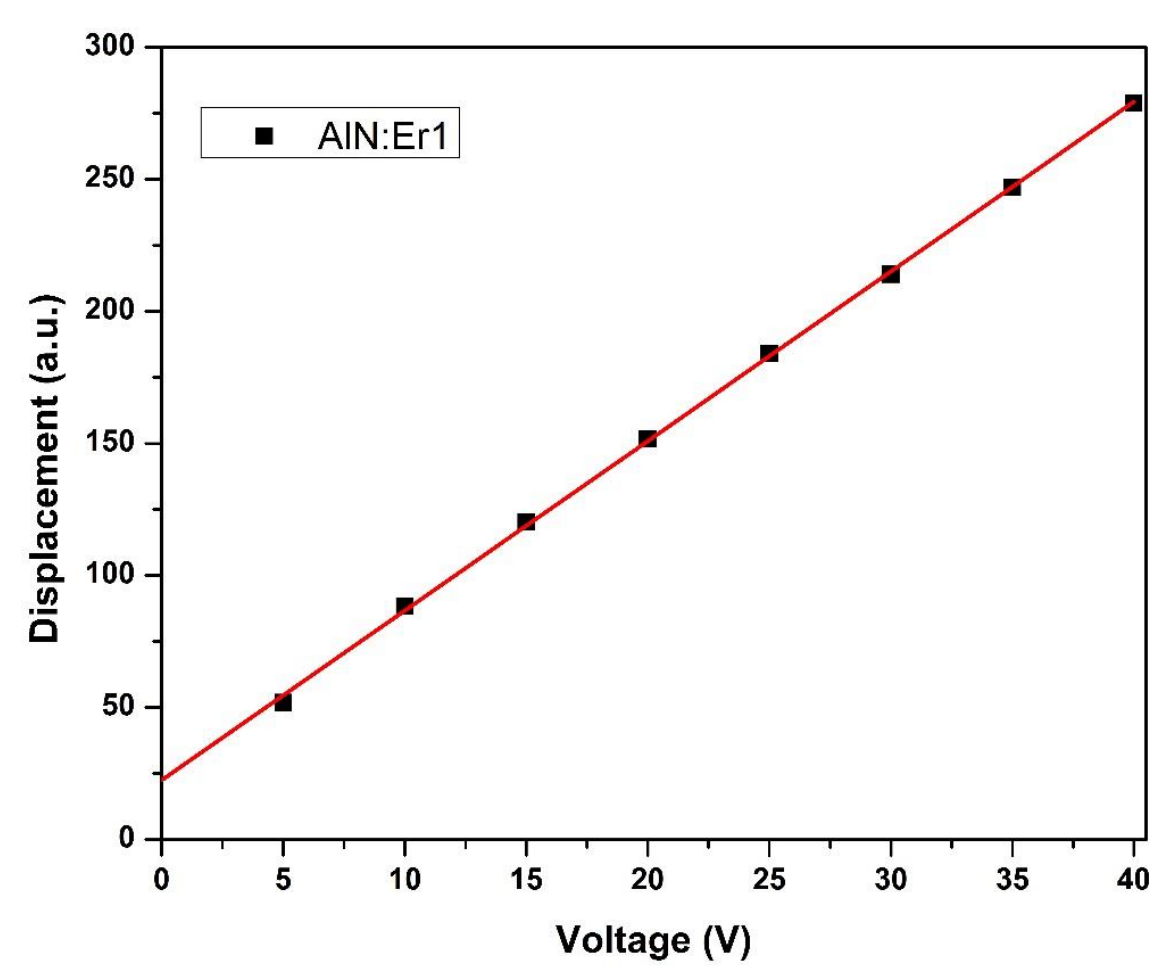

Figure 3.5: Displacement as function of amplitude $\mathrm{V}$ of the ac signal.

In Fig. 3.6, the plots of the refractive index and the piezoelectric coefficient $d_{33}$ for AlN:Er (3\%) thin films prepared using sputtering power between $100 \mathrm{~W}$ and $250 \mathrm{~W}$ are shown. There is a near linear relationship between refractive index and the magnitude of $\mathrm{d}_{33}$ suggesting that better quality of the films leads to higher magnitude of $d_{33}$. This shows that poly-crystallinity of the thin films plays an important role in its piezoelectric properties.

In the measurements shown in Fig. 3.6, the thicknesses of the films was held constant. To see how the thickness of the AlN:Er films affects the measured value of $\mathrm{d}_{33}$, AlN:Er thin films of various thicknesses were deposited at sputter target power of $200 \mathrm{~W}$ and $\mathrm{d}_{33}$ coefficient measurements were carried out using the methodology described above. The results are shown in Figs. 3.7, 3.8 and 3.9 for Er concentrations of 1, 3 and 4 respectively along with the results on pure AlN film The initial measurements of this work in this lab were carried out and reported by Kabulski et al. [45]. For AlN:Er1, the value of $d_{33}$ is larger for thickness only below $400 \mathrm{~nm}$. For larger thickness, $d_{33}$ drops to values lower than that of AlN thin films. The values of $d_{33}$ also has a larger variation over the surface of thin films, as shown in by large error bars. For AlN:Er3 and AlN:Er4 thin films, the magnitude of $d_{33}$ increases as the thickness is increased and the values are larger compared to AlN. The largest $d_{33}$ is obtained for AlN:Er3 thin films, which for some thicknesses show more than two-fold increment in $\mathrm{d}_{33}$ compared to pure AlN. 


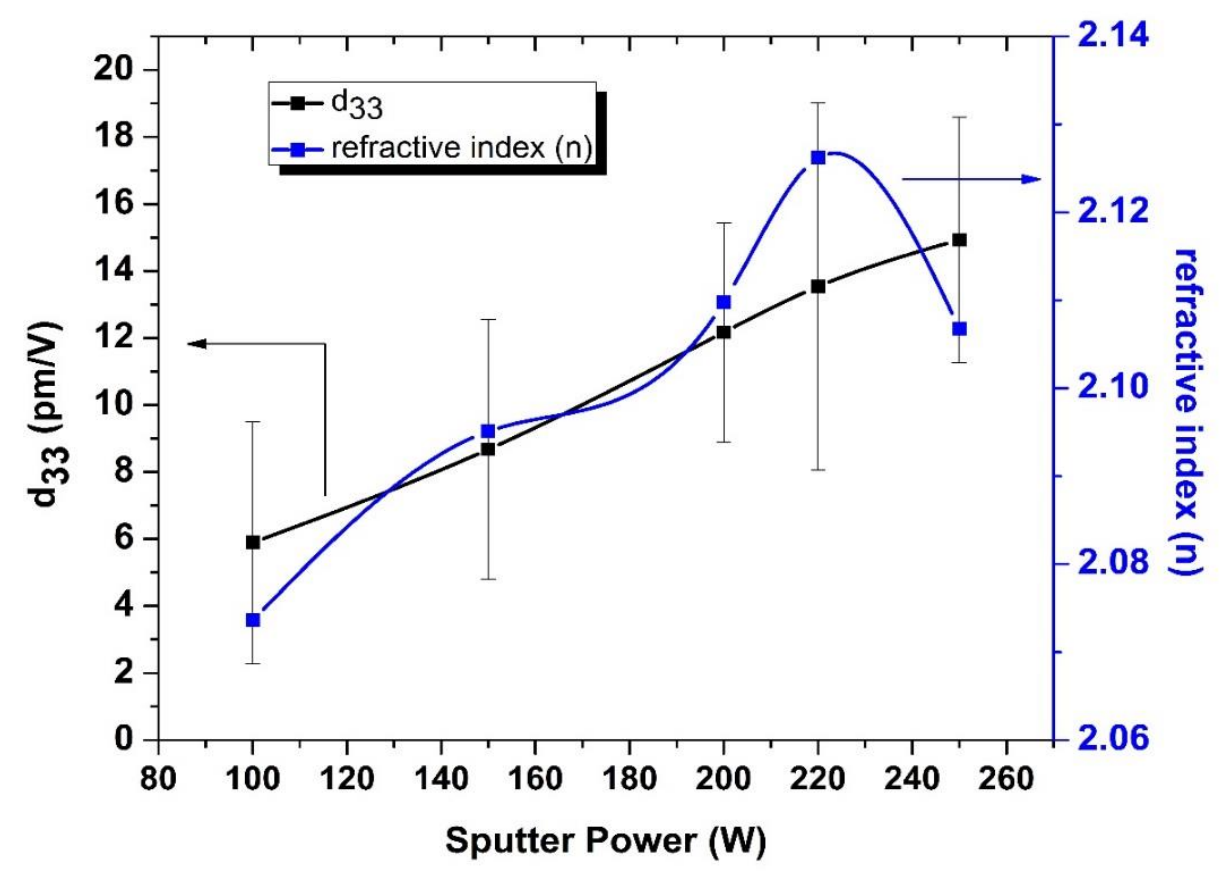

Figure 3.6: Plots of $\mathrm{d}_{33}$ and refractive index for $\mathrm{AlN}$ : $\mathrm{Er}(3 \%)$ films as function of sputtering target power. The lines connecting the data points are visual guides.

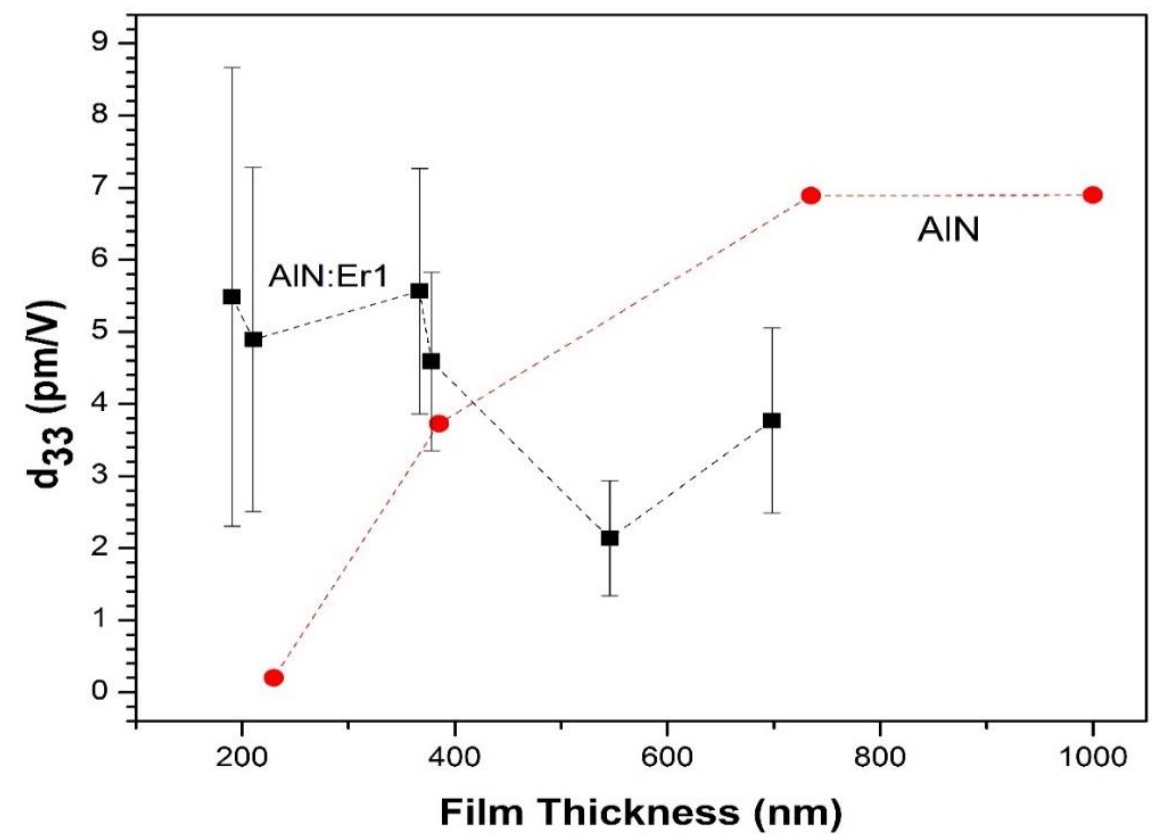

Figure 3.7: $\mathrm{d}_{33}$ vs thickness for AlN:Er1 and AlN thin films. The lines connecting the data points are visual guides. 


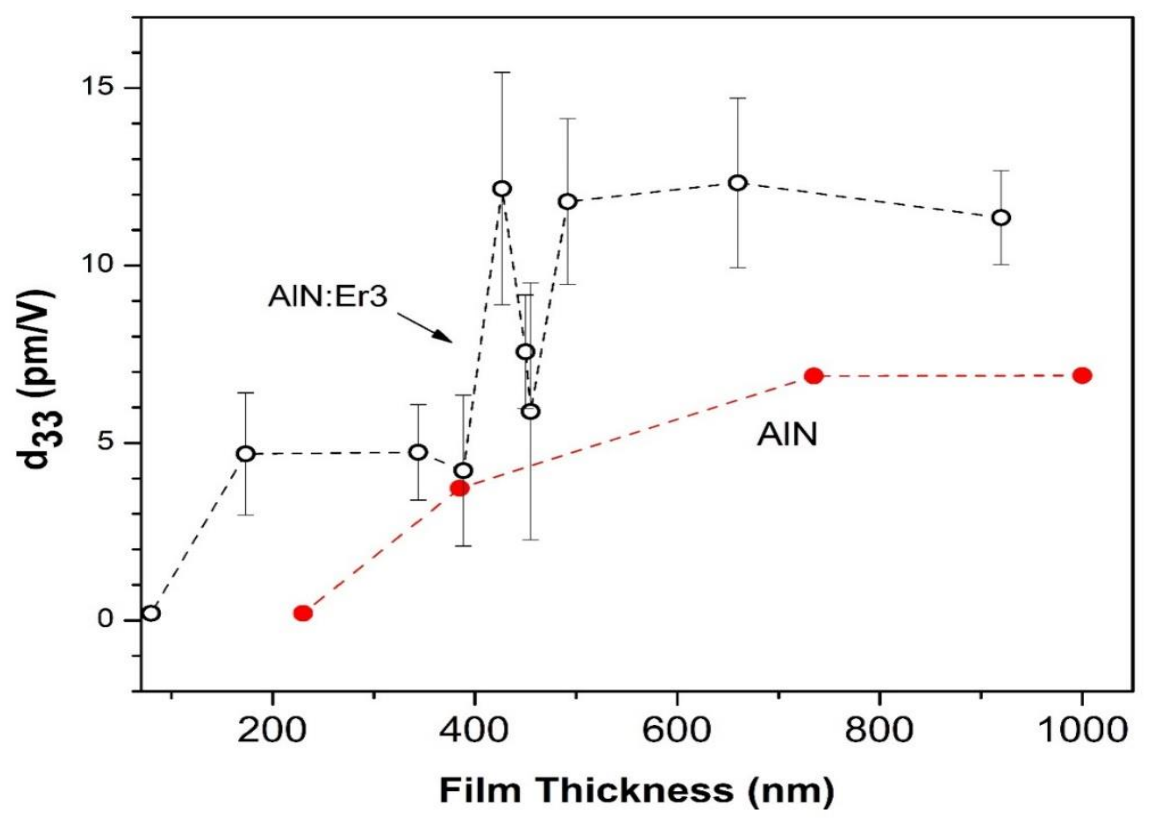

Figure 3.8: $\mathrm{d}_{33}$ vs thickness for AlN:Er3 and AlN thin films. The lines connecting the data points are visual guides.

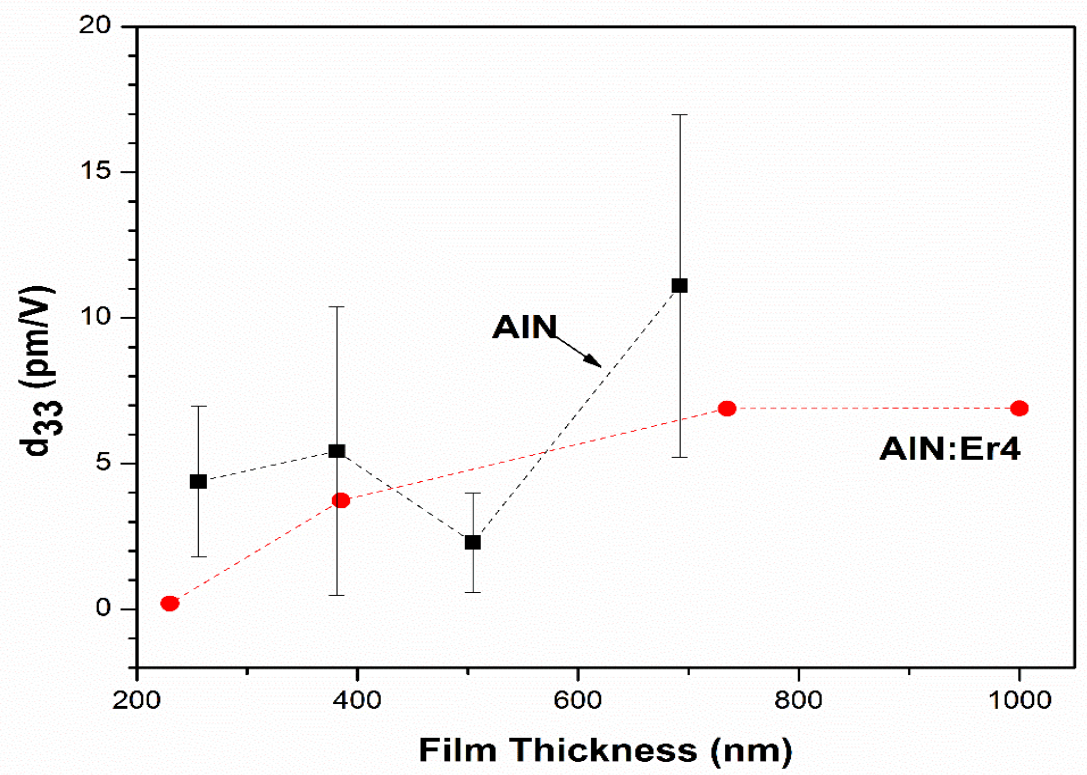

Figure 3.9: $d_{33}$ vs thickness for AlN:Er4 and AlN thin films. The lines connecting the data points are visual guides. 


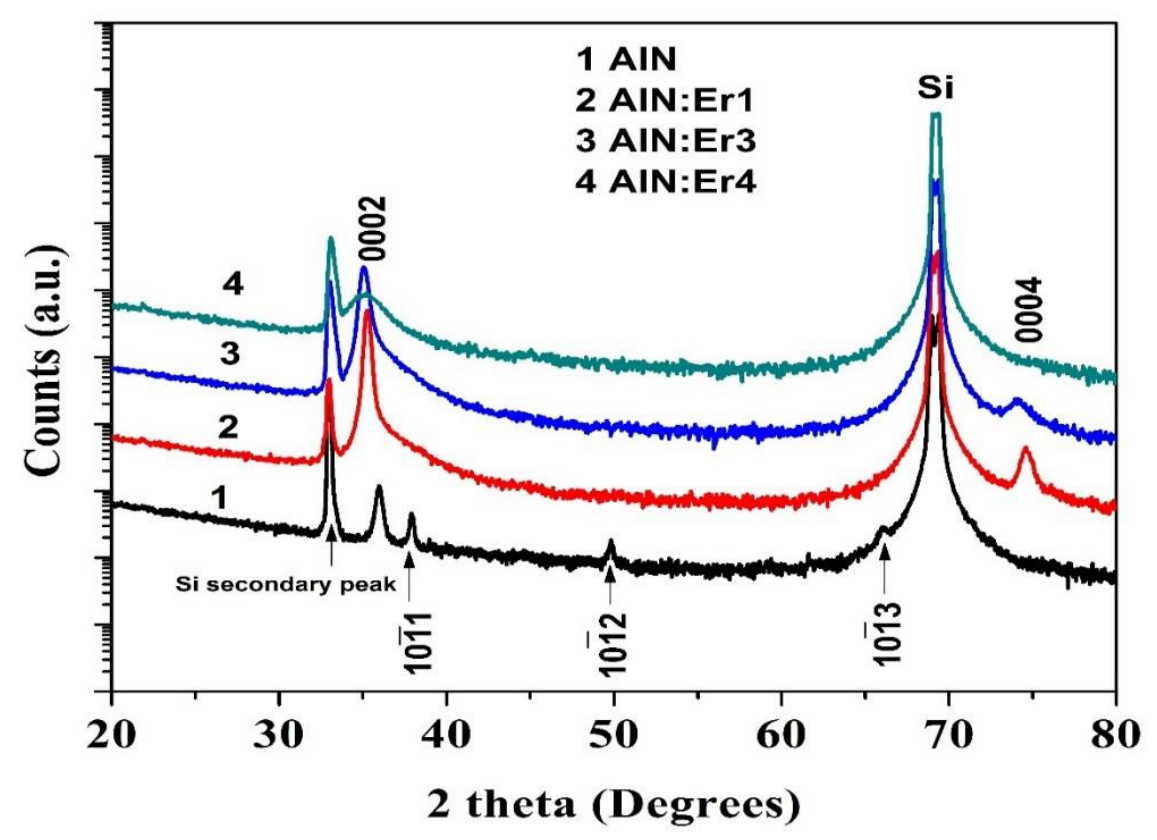

Figure 3.10: XRD pattern for un-annealed AIN and AlN:Er thin films.

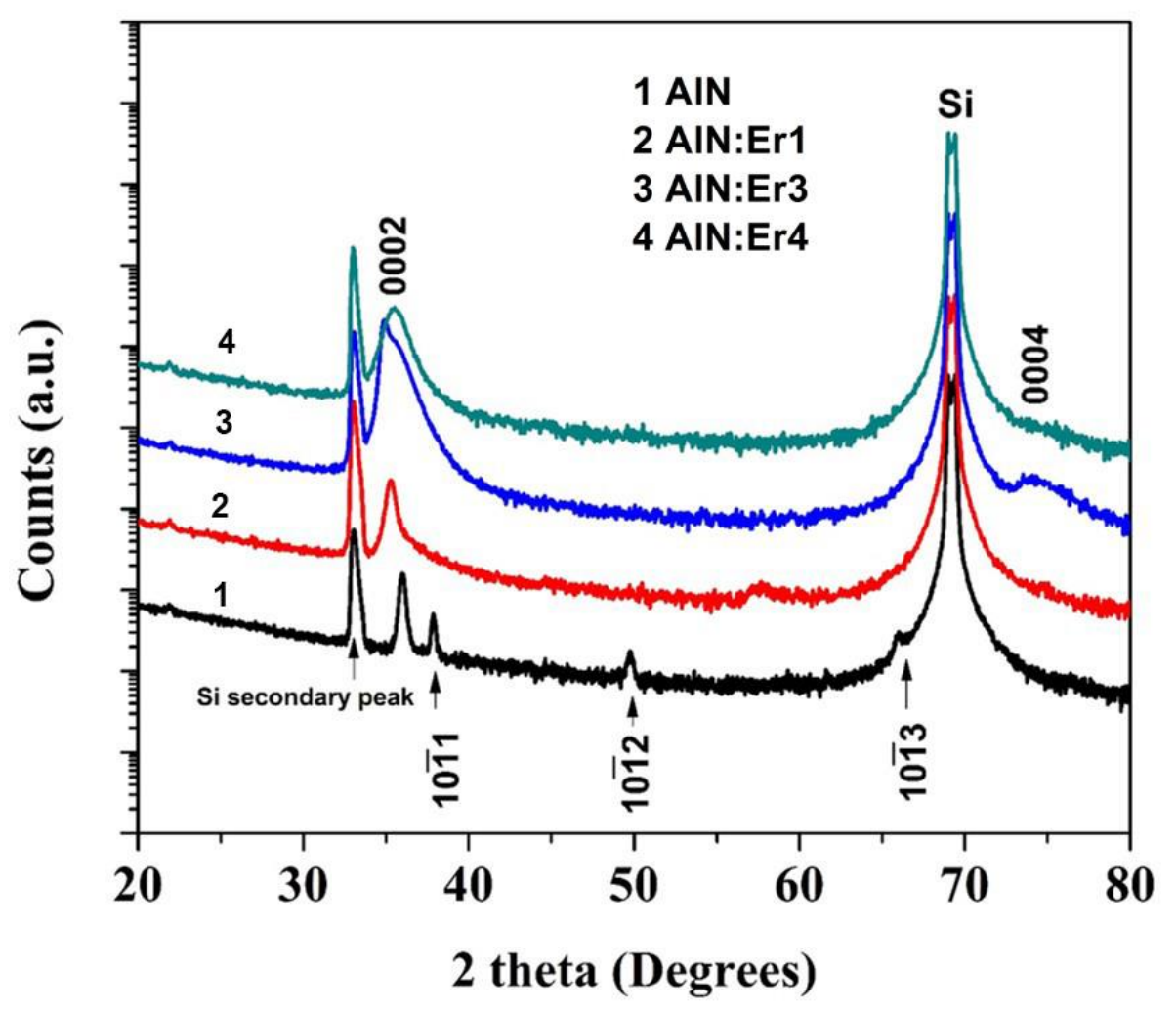

Figure 3.11: XRD pattern for annealed AlN and AlN:Er thin films. 


\subsection{Effect of Annealing on the X-ray Diffraction Patterns of AIN:Er Thin Films:}

X-ray diffraction measurements performed on the thin films are shown in Figure 3.10. As discussed in Chapter 2, for the AlN film, the observed peaks can be assigned to either AlN or the Si-

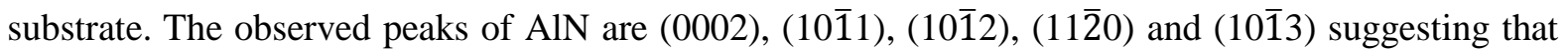
the AlN film has no preferential orientation. The peak at $33^{\circ}$ is the second harmonic of the $\mathrm{Si}(004)$ peak, resulting due to the Bremsstrahlung effect. For the AlN:Er film, only the (0002) and the (0004) peaks are observed besides the Si peaks and the lines (10 $\overline{1} 1),(10 \overline{1} 2),(11 \overline{2} 0)$ and (10 $\overline{1} 3)$ are absent suggesting preferential orientation of the film perpendicular to the c-axis after $\mathrm{Er}$ is introduced into the film. This preferential orientation of AlN:Er is considered to be an important reason for the several fold enhancement of the piezoelectric coefficient of AlN:Er thin films compared to Er free AlN films. XRD patterns of the films annealed at $600{ }^{\circ} \mathrm{C}$ in air for 45 minutes are shown in Fig. 3.11. The films retained their lattice structure after annealing since there is no difference in the x-ray diffraction patterns of Fig. 3.10 and Fig. 3.11. Hence it can be inferred that the magnitude of $d_{33}$ is not affected by heat treatment of the films at $600{ }^{\circ} \mathrm{C}$ in air. This is important for the applications of these films for temperatures up to at least $600{ }^{\circ} \mathrm{C}$. 


\section{CHAPTER 4 - Magnetic Characterization of the Electronic State of Er in AIN:Er Films}

\subsection{Introduction:}

In this chapter, results of magnetic measurements on Er metal and AlN:Er are presented in order to determine the electronic state of $\mathrm{Er}$ in AlN:Er. Although detailed studies of the magnetic properties of $\mathrm{Er}_{2} \mathrm{O}_{3}$ are presented in Chapter $\mathrm{V}$, some comparison is also given in this chapter to clarify the electronic state of Er in AlN:Er. For proper discussion of the measured magnetic properties, a brief overview of the basic theories of magnetism is first presented below based on the material given in the books by Kittel [37] and Morrish [46]. Experimental results presented in this chapter have appeared in print in a publication by the author [47].

\subsection{Curie Law, Curie-Weiss Law and Exchange Interaction:}

\subsubsection{Curie Law:}

An electron has orbital angular momentum $\vec{L}$ due to its orbital motion and spin angular momentum $\vec{S}$ due to its spin. The magnetic moment of an electron arising from the orbital angular momentum and spin angular momentum are given by: $\overrightarrow{\mu_{l}}=\gamma_{l} \vec{L}$ and $\overrightarrow{\mu_{s}}=\gamma_{s} \vec{S}$ where $\gamma_{l}$ and $\gamma_{s}$ are orbital and spin gyromagnetic ratios for the electron. The gyromagnetic ratios are defined as: $\gamma_{L}=\frac{g_{l} \mu_{B}}{\hbar}$ and $\gamma_{S}=\frac{g_{S} \mu_{B}}{\hbar}$ where quantity $\mathrm{g}$ is called $\mathrm{g}$ factor. For orbital angular momentum $\mathrm{g}_{l}=1$ and for spin $\mathrm{g}_{s}$ $=2.0023$ which is usually taken as 2.00. $\mu_{B}$ is Bohr magneton and is equal to $e \hbar / 2 m c$ in CGS units and $e \hbar / 2 m$ in SI units. Since charge e on an electron is negative, magnetic moment and angular momentum are antiparallel to each other.

If an electron has both orbital and spin components, then the total angular momentum $\vec{J}$ is given by $\vec{J}=\vec{L}+\vec{S}$ leading to

$$
\vec{\mu}=g_{L} \mu_{B} \vec{J} / \hbar
$$

$$
\text { with } \quad g_{L}=1+\frac{J(J+1)+S(S+1)-L(L+1)}{2 J(J+1)}
$$

Here $\mathrm{g}_{\mathrm{L}}$ is called Landé $\mathrm{g}$-factor. When magnetic field $\mathrm{H}$ is applied, the Hamiltonian $\mathcal{H}$ is given by

$$
\mathcal{H}=-\vec{\mu} \cdot \vec{H}=\frac{g\left|\mu_{B}\right|}{\hbar} \vec{H} \cdot \vec{J}
$$


If the applied field is in z-direction, then with $\mu_{B}$ positive:

$$
\mathcal{H}=\frac{g \mu_{B}}{\hbar} H J_{Z}
$$

In the applied magnetic field, instead of $\mathrm{n}, 1, \mathrm{~m}_{\mathrm{l}}$ and $\mathrm{m}_{\mathrm{s}}, \mathrm{n}, \mathrm{l}, \mathrm{J}$ and $\mathrm{m}_{\mathrm{J}}$ are the good quantum numbers with $|\mathrm{J}|=|\mathrm{L}-\mathrm{S}|,|\mathrm{L}-\mathrm{S}+1|, \ldots,|\mathrm{L}+\mathrm{S}|$. Also for a given $\mathrm{J}, \mathrm{m}_{\mathrm{J}}=-\mathrm{J},-\mathrm{J}+1, \ldots \ldots$ to $\mathrm{J}-1, \mathrm{~J}$ and energy of a state with quantum numbers $\mathrm{J}$ and $\mathrm{m}$ is given by:

$$
E_{J m}=\left\langle j m_{j}{ }^{\prime}|\mathcal{H}| j m_{j}\right\rangle=g \mu_{B} m_{j} H
$$

The free energy of a system with energy levels $E_{n}$ in terms of partition function $Z$ is given by:

$$
F=-k_{B} T \ln (Z)=-k_{B} T \ln \sum_{n} e^{-E_{n} / k_{B} T}
$$

The magnetization $\mathrm{M}$ of the system with $\mathrm{N}$ electrons is given by:

$$
M=-N \frac{\partial F}{\partial H}=-N \frac{\sum_{n} \frac{\partial E_{n}}{\partial H} e^{-E_{n} / k_{B} T}}{\sum_{n} e^{-E_{n} / k_{B} T}}
$$

From Eq. (4.5), we have

$$
\frac{\partial E_{n}}{\partial H}=g \mu_{B} m_{J}
$$

Using this in Eq. (4.7) gives

$$
M=-N g \mu_{B} \frac{\sum_{-J}^{+J}\left(m e^{-g \mu_{B} m H / k_{B} T}\right)}{\sum_{-J}^{+J}\left(e^{-g \mu_{B} m H / k_{B} T}\right)} \text { with } m_{J}=-\mathrm{J},-\mathrm{J}+1, \ldots . \text { to } \mathrm{J}-1, \mathrm{~J}
$$

Substituting $x=g \mu_{B} m / k_{B} T$

$$
\begin{gathered}
M=-N g \mu_{B} \frac{\sum_{-J}^{+J}\left(m e^{-x m_{J}}\right)}{\sum_{-J}^{+J}\left(e^{-x m_{J}}\right)} \\
M=N g \mu_{B} J\left\{\left(\frac{2 J+1}{2 J}\right) \operatorname{coth}\left[\left(\frac{2 J+1}{2 J}\right) x J\right]-\frac{1}{2 J} \operatorname{coth}\left(\frac{x J}{2 J}\right)\right\}
\end{gathered}
$$

or 


$$
M=N g \mu_{B} J \cdot B_{J}(y)
$$

where $y \equiv x J \equiv g \mu_{B} H J / k_{B} T$ and $B_{J}(y)=\frac{2 J+1}{2 J} \operatorname{coth}\left(\frac{2 J+1}{2 J}\right) y-\frac{1}{2 J} \operatorname{coth}\left(\frac{y}{2 J}\right)$ is called the Brillouin function.

For $y \ll 1, B_{J}(y)=\frac{1}{3} \frac{(J+1)}{J} y$. In this case, it can be shown that Eq. (4.11) reduces to

$$
\begin{gathered}
M=\frac{N g^{2} J(J+1) \mu_{B}^{2} H}{3 k_{B} T}=\frac{C H}{T} \\
C=\frac{N g^{2} J(J+1) \mu_{B}{ }^{2}}{3 k_{B}}
\end{gathered}
$$

And then magnetic susceptibility,

$$
\chi=\frac{\partial M}{\partial H}=\frac{N g^{2} J(J+1) \mu_{B}^{2}}{3 k T}=\frac{N \mu^{2}}{3 k T}
$$

where $\mu=g \mu_{B} \sqrt{J(J+1)}$ and with $C=\frac{N \mu^{2}}{3 k}$, Eq. (4.14) becomes:

$$
\chi=\frac{C}{T}
$$

Eq. (4.15) is called the Curie's law and $C=\frac{N \mu^{2}}{3 k}$ is called the Curie constant whose magnitude is used to determine the effective magnetic moment $\mu$ of the electron in a material.

\subsubsection{Curie-Weiss Law:}

The Curie law given by Eq. (4.15) is not sufficient to explain why materials become magnetically ordered at high temperatures. For example, iron orders ferromagnetically at $\mathrm{T}_{\mathrm{C}}=1040 \mathrm{~K}$ where $\chi$ diverges. In order to explain such cases, Weiss in 1905 proposed that in addition to an applied field, magnetic interactions between the electrons produces an internal magnetic field equal to $\gamma \mathrm{M}$. This modifies the Curie law (Eq. (4.15)) as follows:

$$
M=\frac{C(H+\gamma M)}{T}=\frac{C H}{T-\gamma C}=\frac{C H}{T-\theta}
$$


Eq. (4.16) is known as Curie-Weiss law. Constants $C$ and $\theta=\gamma \mathrm{C}$ are determined experimentally using this equation, as shown later in this chapter. At $\mathrm{T}_{\mathrm{C}}=\theta=\gamma \mathrm{C}, \chi$ diverges leading to magnetic ordering of the system. The source of the internal magnetic field or Weiss field became understood only after Quantum Mechanics was developed and this source is the Heisenberg-van Vleck-Dirac exchange interaction briefly discussed below.

\subsubsection{Heisenberg Exchange Interaction:}

The Hamiltonian for interaction between two neighboring spins $\overrightarrow{S_{a}}$ and $\overrightarrow{S_{b}}$, known as Heisenberg exchange interaction, is given by:

$$
\mathcal{H}_{e x}=-2 J_{a b} \overrightarrow{S_{a}} \cdot \overrightarrow{s_{b}}
$$

where $\mathrm{J}_{\mathrm{ab}}$ is exchange integral. If there are $\mathrm{Z}$ nearest neighbors among which the exchange is nonzero, then:

$$
\left\langle\mathcal{H}_{e x}\right\rangle=-2 \sum_{a \neq b}^{n n} J_{a b} \overrightarrow{S_{a}} \cdot \overrightarrow{s_{b}}
$$

or

$$
\left\langle\mathcal{H}_{e x}\right\rangle=-2 J_{a b}\left\langle\overrightarrow{S_{a}}\right\rangle \sum_{b=1}^{Z}\left\langle\overrightarrow{s_{b}}\right\rangle
$$

If $\mathrm{J}_{\mathrm{ab}}$ is same for all the nearest neighbors, $\mathrm{J}_{\mathrm{ab}}=\mathrm{J}_{\mathrm{e}}$, the exchange interaction. Using $\mathrm{H}=\gamma \mathrm{M}$, it can be shown that

$$
\gamma=\frac{2 J_{e} Z}{N g^{2} \mu_{B}^{2}}
$$

This leads to

$$
\theta=\gamma C=\frac{2 J_{e} Z J(J+1)}{3 k_{B}}
$$

Eqs. (4.18) and (4.19) show that the critical ordering temperature $\theta$ (also called the Curie temperature $\mathrm{T}_{\mathrm{C}}$ ) and $\gamma$, the Weiss molecular filed constant, are directly proportional to $\mathrm{J}_{\mathrm{e}}$, the exchange constant. In Eq. (4.17), a positive $\mathrm{J}_{\mathrm{e}}$ leads to a ferromagnetic ground state in which the spins are parallel. On the other hand, if $\mathrm{J}_{\mathrm{e}}$ is negative, then the ground state has antiparallel spins leading to antiferromagnetism and $\theta$ in the Curie-Weiss law, Eq. (4.16), is negative. Neel predicted antiferromagnetism which was later confirmed in $\mathrm{MnO}$ by neutron diffraction measurements. In his honor, the magnetic ordering temperature in antiferromagnets is called the Neel temperature $\mathrm{T}_{\mathrm{N}}$. For $\mathrm{MnO}, \mathrm{T}_{\mathrm{N}}=118 \mathrm{~K}$. 


\subsection{Magnetic Measurements:}

The three possible states of Er in AlN:Er are (i) Er present as elemental Er; (ii) Er present as $\mathrm{Er}_{2} \mathrm{O}_{3}$ since the presence of oxygen is detected by XPS; and (iii) Er present as $\mathrm{Er}^{3+}$ substituting for $\mathrm{Al}^{3+}$. The magnetic measurements were used to distinguish between the three possibilities. For this purpose, temperature dependence of the magnetization $(\mathrm{M})$ in a fixed magnetic field $(\mathrm{H})$ was measured at temperatures between $2 \mathrm{~K}$ and $300 \mathrm{~K}$ and isothermal measurements of $\mathrm{M}$ versus $\mathrm{H}$ at suitable temperatures in $\mathrm{H}$ up to $90 \mathrm{kOe}$ for the films of $\mathrm{AlN}$ and $\mathrm{AlN}$ :Er, for elemental Er and for a powder sample of $\mathrm{Er}_{2} \mathrm{O}_{3}$. In these cases, temperature versus magnetization measurements were done for the zero-field-cooled (ZFC) and field-cooled (FC) cases. For ZFC, the samples are cooled in $\mathrm{H}=0$ to $2 \mathrm{~K}$, a suitable magnetic field is then applied and data taken with increasing temperatures by stabilizing the temperature at each point. After the temperature has reached $300 \mathrm{~K}$, the data is then taken with decreasing temperatures in the same $\mathrm{H}$ resulting in the FC data. This data for AlN:Er films and Er metal are presented and analyzed in this chapter. Detailed analysis of measurements on $\mathrm{Er}_{2} \mathrm{O}_{3}$ samples is presented in Chapter 5.

\subsubsection{Magnetic Measurements on Er Metal:}

Erbium crystallizes in the hcp (hexagonal closed-packed) structure with lattice constant $\mathrm{a}=$ $0.3559 \mathrm{~nm}$ and $\mathrm{c}=0.5585 \mathrm{~nm}$. The electronic arrangement for the outer shells for $\mathrm{Er}^{3+}$ ion is $4 \mathrm{f}^{11} 5 \mathrm{~s}^{2} 5 \mathrm{p}^{6}$ so that the partially filled $4 \mathrm{f}$ shell is shielded by the outer covers of $5 \mathrm{~s}^{2} 5 \mathrm{p}^{6}$ shells. This makes $\mathrm{J}$ as a good quantum number. Using Hunds' rules, this gives $\mathrm{L}=6, \mathrm{~S}=3 / 2$ and $\mathrm{J}=15 / 2$ for $\mathrm{Er}^{3+}$ ion with ${ }^{4} \mathrm{I}_{15 / 2}$ as the ground state. Using Eq. (4.2), the Lande $\mathrm{g}_{\mathrm{L}}=6 / 5$ and magnetic moment $\mu=\mathrm{g}_{\mathrm{L}} \mathrm{J} \mu_{B}=9 \mu_{B}$ for $\mathrm{Er}^{3+}$ is expected.

The data of the magnetic susceptibility $(\mathrm{M} / \mathrm{H})$ vs. T for Er metal is presented in Fig. 4.1. The data shows a weak peak near $85 \mathrm{~K}$ and an increase in the magnetization near $30 \mathrm{~K}$ with noticeable irreversibility between the $\mathrm{FC}$ and $\mathrm{ZFC}$ data below $30 \mathrm{~K}$. The $\mathrm{d}(\chi \mathrm{T}) / \mathrm{dT}$ vs $\mathrm{T}$ plot is shown in Fig. 4.2 since it is known $[48,49]$ that the temperature variation of $\mathrm{d}(\chi \mathrm{T}) / \mathrm{dT}$ resembles that of the specific heat near a magnetic transition. The magnetic transitions in Er have been reported by Green et al. and Habenschuss et al. studied magnetic structure of Er by neutron diffraction [50, 51]. According to their measurements, above $85 \mathrm{~K}$ the Er metal is in paramagnetic state. As the temperature is lowered, the moments along c-axis become ordered in sinusoidal manner. This state in Fig 4.2 is denoted as c-axis modulated (CAM). The basal plane components of magnetic moment at $\mathrm{T}=85 \mathrm{~K}$ are still disordered. As the temperature is lowered, at $53 \mathrm{~K}$ basal plane components align in helical order. In this state, Erbium has several layers with c-component in upward direction, which is followed by equal number 
of layers with c-component in downward direction. At $\mathrm{T}=35 \mathrm{~K}$, Erbium magnetic structure becomes commensurate and at $\mathrm{T} \simeq 20 \mathrm{~K}$ it transitions to conical ferromagnetic state.

The magnetic measurements reported here on Erbium metal are shown in Fig. 4.1 with additional analysis of the data shown in Figs. 4.2 and 4.3 are consistent with the magnetic transitions reported by Legvold [52] and Habenschuss et al. [50]. The transition at $85 \mathrm{~K}$ is clearly evident in Figs. 4.1 and 4.2 and the positions of the transitions at $53 \mathrm{~K}$ and $30 \mathrm{~K}$ are also marked in Fig. 4.2. The data above $85 \mathrm{~K}$ is analyzed in terms of Curie-Weiss variation and is shown in Figure 4.3. The calculated values of $\mathrm{C}$ and $\theta$ are shown in Fig. 4.3. From the magnitude of $\mathrm{C}$ and Eq. (4.13), the magnetic moment $\mu=9.46 \mu_{\mathrm{B}}$ is calculated close to the expected value of $\mu$ for Er metal.

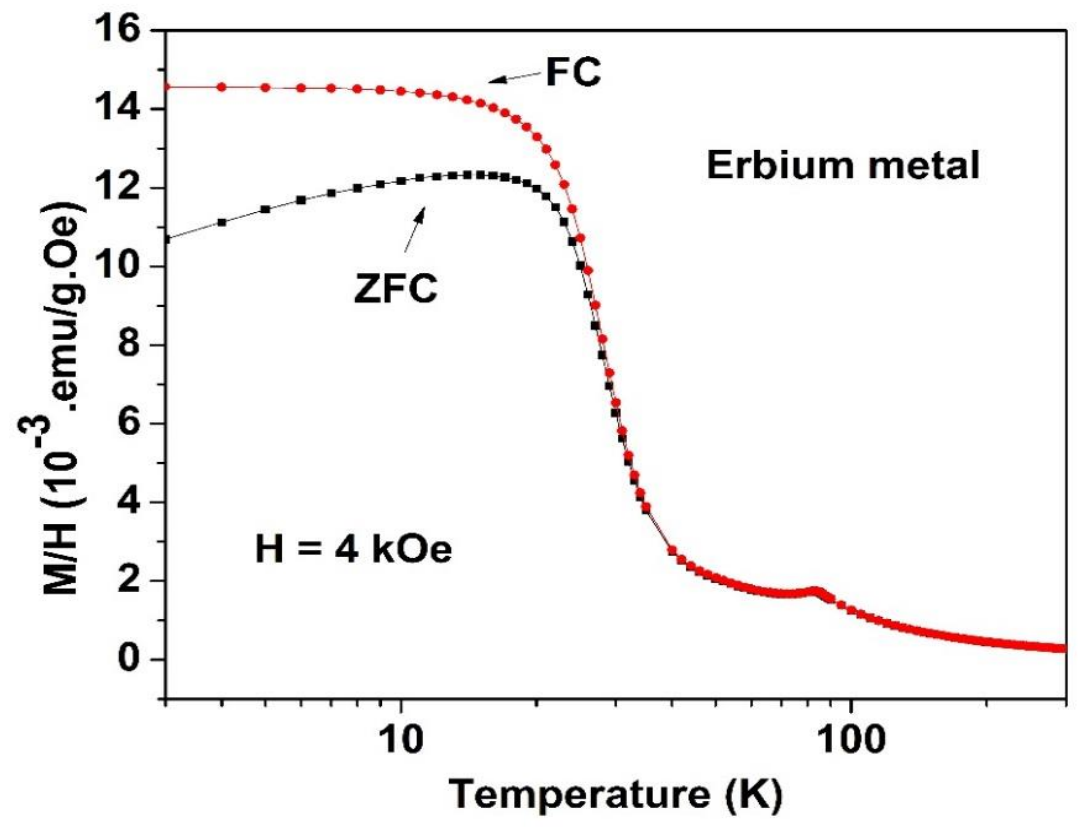

Figure 4.1: Temperature dependence of the magnetic susceptibility $\chi=\mathrm{M} / \mathrm{H}$ of Er metal in the temperature range of 2 to $300 \mathrm{~K}$ under $\mathrm{ZFC}$ and $\mathrm{FC}$ conditions. 


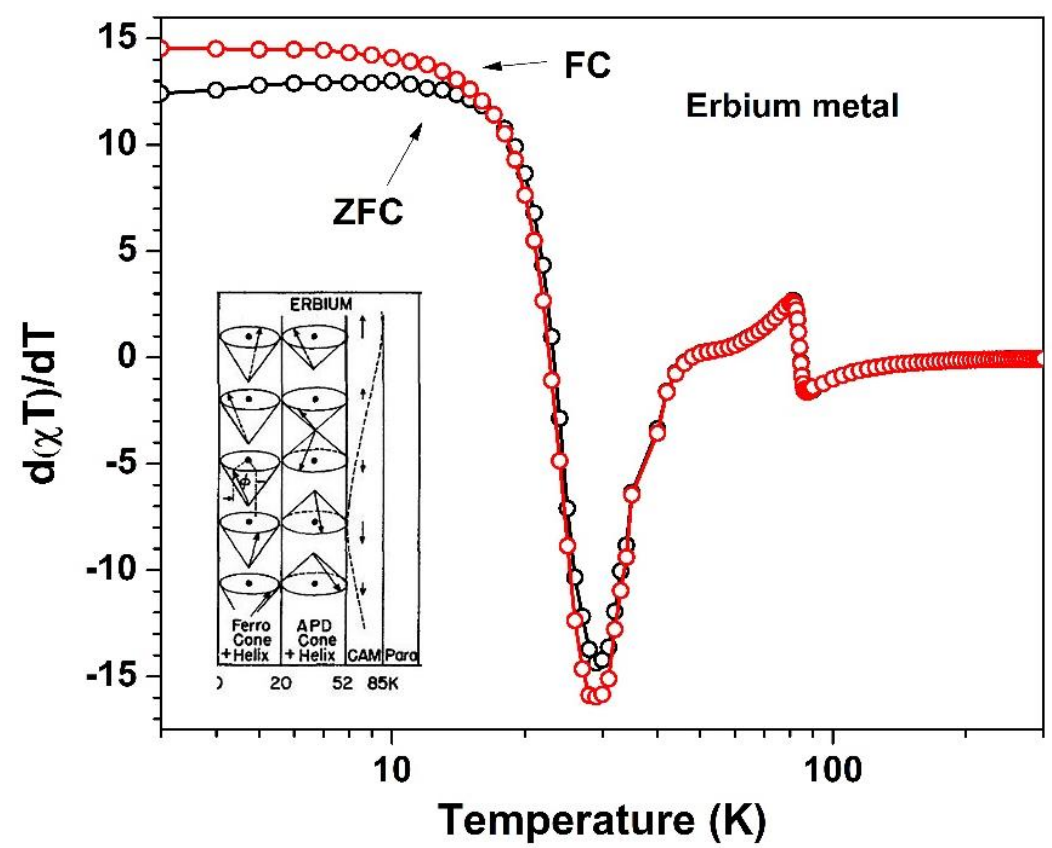

Figure 4.2: Plots of computed $\mathrm{d}(\chi \mathrm{T}) / \mathrm{dT}$ from $\mathrm{ZFC}$ and $\mathrm{FC}$ data of Erbium metal to determine various transition temperatures when applied $\mathrm{H}=4000$ Oe.

In order to get additional information on the nature of different magnetic phases of Er, M vs. $\mathrm{H}$ measurements were performed on the Erbium metal sample at three different temperatures; $\mathrm{T}=5 \mathrm{~K}$, $50 \mathrm{~K}$ and $110 \mathrm{~K}$, each value of temperature falls into three different regions of erbium's magnetic state (paramagnetic at $\mathrm{T}=110 \mathrm{~K}$, antiferromagnetic for $\mathrm{T}=50 \mathrm{~K}$ and ferromagnetic state for $\mathrm{T}=5 \mathrm{~K}$ ) as determined from ZFC and FC measurements. As expected, measurement at $\mathrm{T}=5 \mathrm{~K}$ (Fig. 4.4) performed from $\mathrm{H}=90 \mathrm{kOe}$ to $\mathrm{H}=-90 \mathrm{kOe}$ to cover the full loop shows hysteresis characteristic of a ferromagnetic state. On the other hand, measurements at $\mathrm{T}=50 \mathrm{~K}$ and $\mathrm{T}=110 \mathrm{~K}$ do not show any hysteresis. The measurements at this temperature for range up to $\mathrm{H}=90 \mathrm{kOe}$ are shown in Figure 4.5. $\mathrm{M}$ vs $\mathrm{H}$ curve for paramagnetic state of $\mathrm{Er}$ at $\mathrm{T}=110 \mathrm{~K}$ is linear as expected for a paramagnet. Since there is a clear change in slope of $\mathrm{M}$ vs $\mathrm{H}$ for the data at $5 \mathrm{~K}$ and $50 \mathrm{~K}, \mathrm{dM} / \mathrm{dH}$ vs $\mathrm{H}$ is calculated and plotted and is shown in Fig. 4.6. At $\mathrm{T}=5 \mathrm{~K}$, the changes in $\mathrm{dM} / \mathrm{dH}$ vs $\mathrm{H}$ are related to hysteresis whereas at $50 \mathrm{~K}$, the changes in $\mathrm{dM} / \mathrm{dH}$ vs $\mathrm{H}$ in particular the peak at $25 \mathrm{kOe}$ is related to the spin flop transition of the antiferromagnetic state, as also noted in the review by Legvold [52]. This spin flop transition is a general feature of the antiferromagnetic ordering when $\mathrm{H}$ is applied along the easy direction [53]. In the spin flop transition, the magnetic moments flop to a direction perpendicular to the applied field yet still remaining antiparallel. 


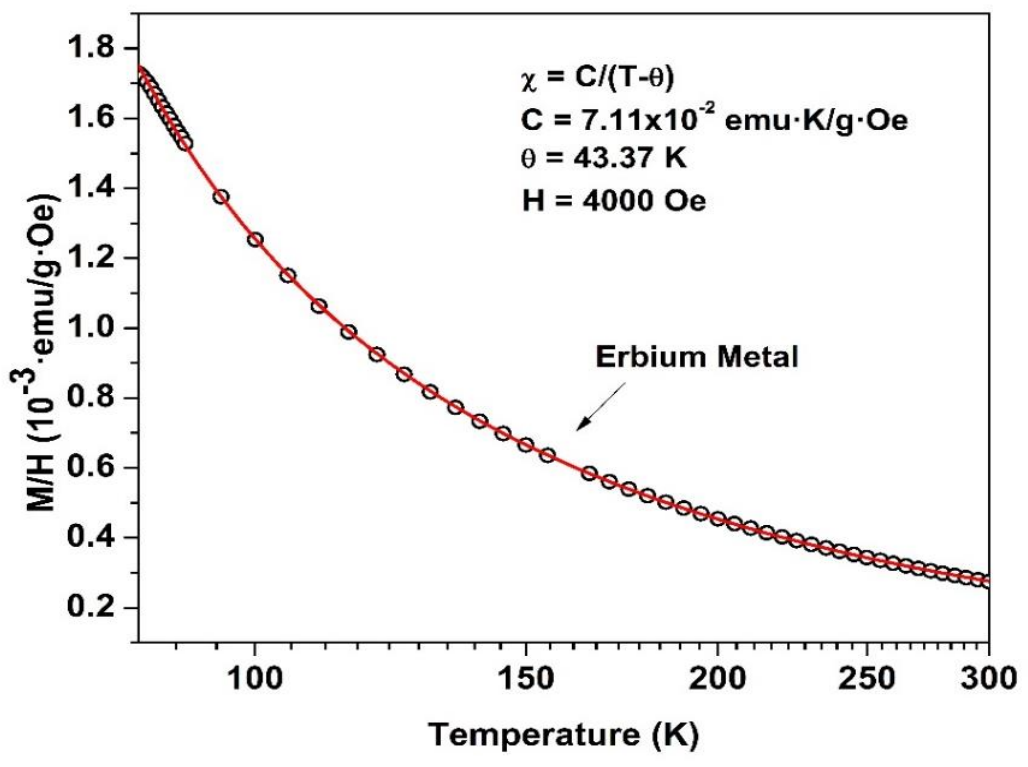

Figure 4.3: Shown are the experimental results on the temperature dependence of the magnetic susceptibility M/H for the Erbium metal with the solid line fit to the Curie-Weiss law (Eq. 4.16) with the parameters of the fit given in the figure.

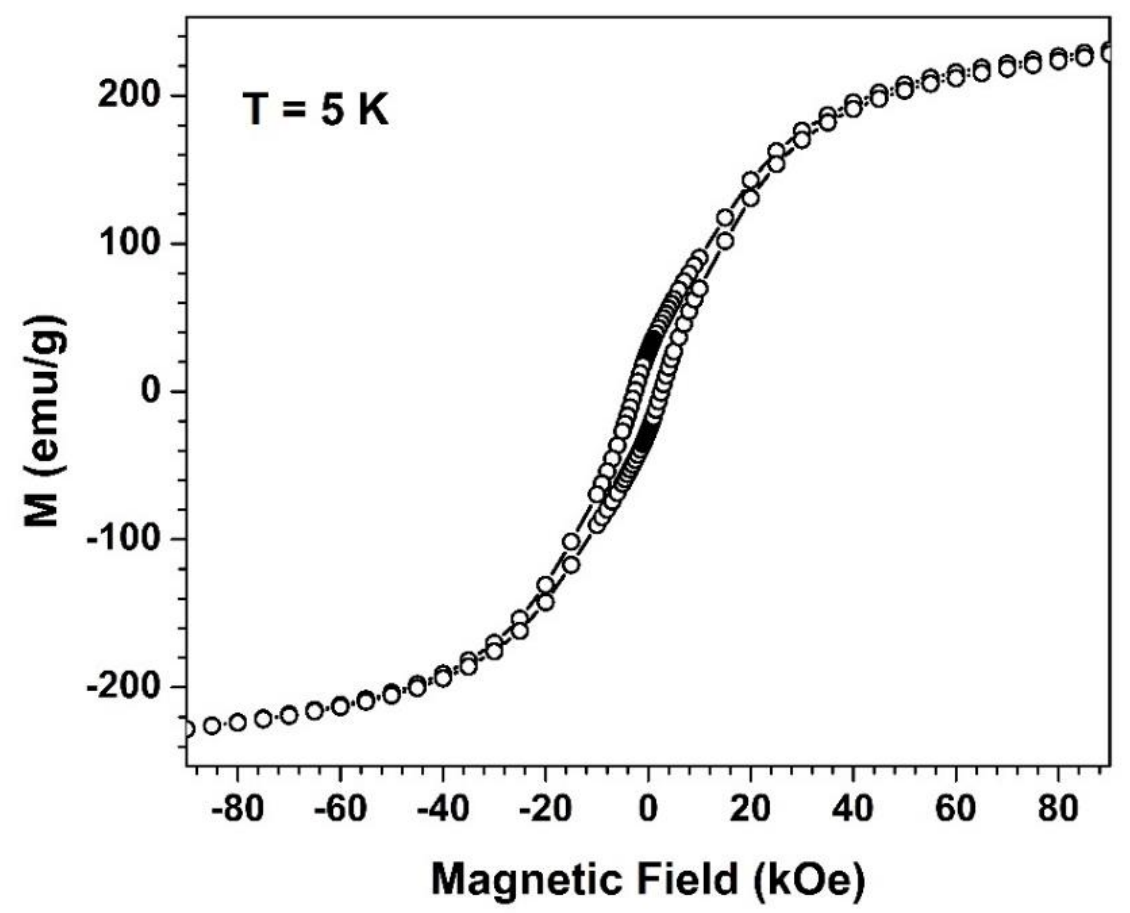

Figure 4.4: Magnetic Field dependence of the magnetization of the Erbium metal at $\mathrm{T}=5 \mathrm{~K}$. 


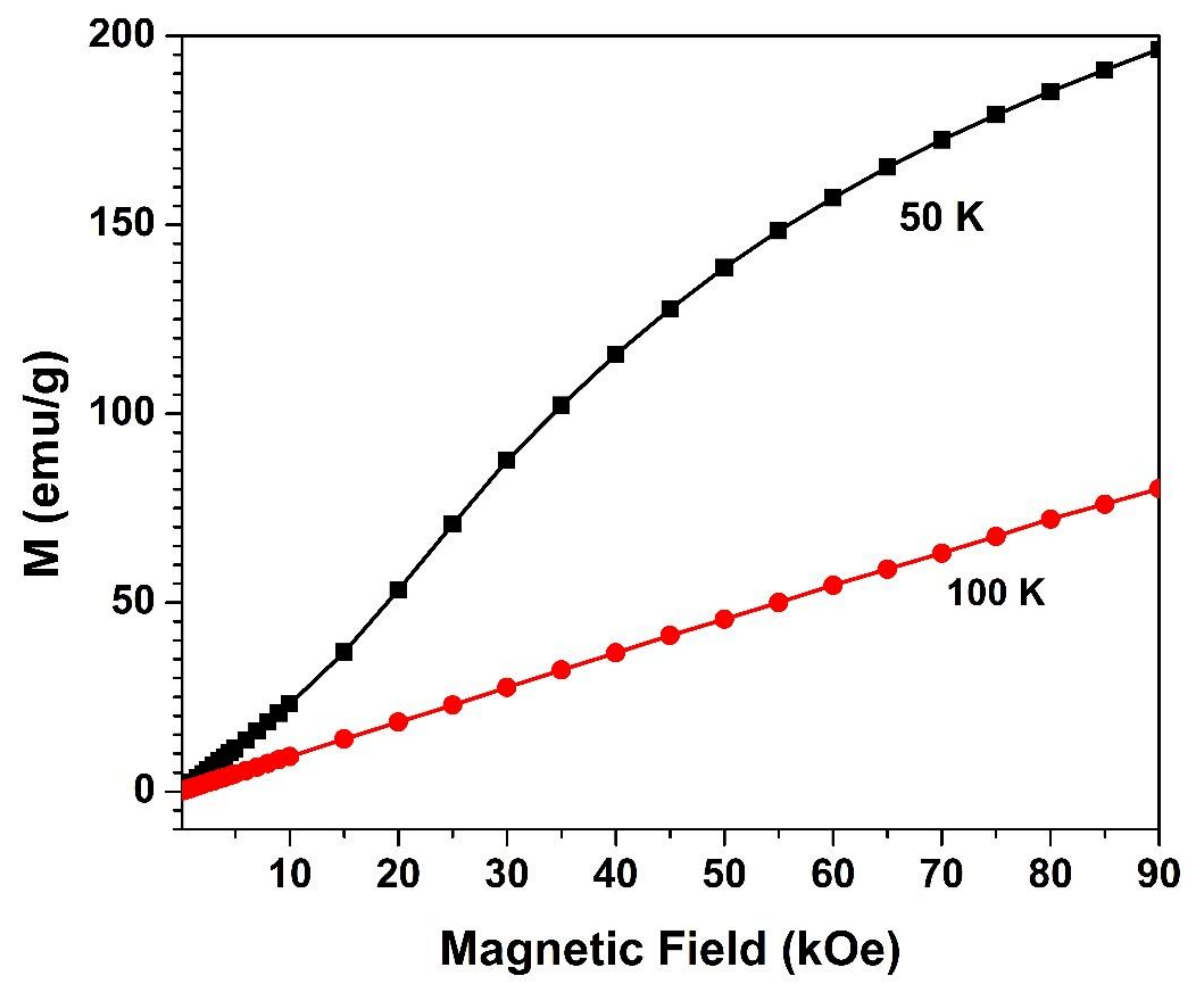

Figure 4.5: Magnetic Field dependence of the magnetization of the Erbium metal at $\mathrm{T}=50 \mathrm{~K}$ and $\mathrm{T}=$ $110 \mathrm{~K}$. The lines connecting the data points are visual guides.

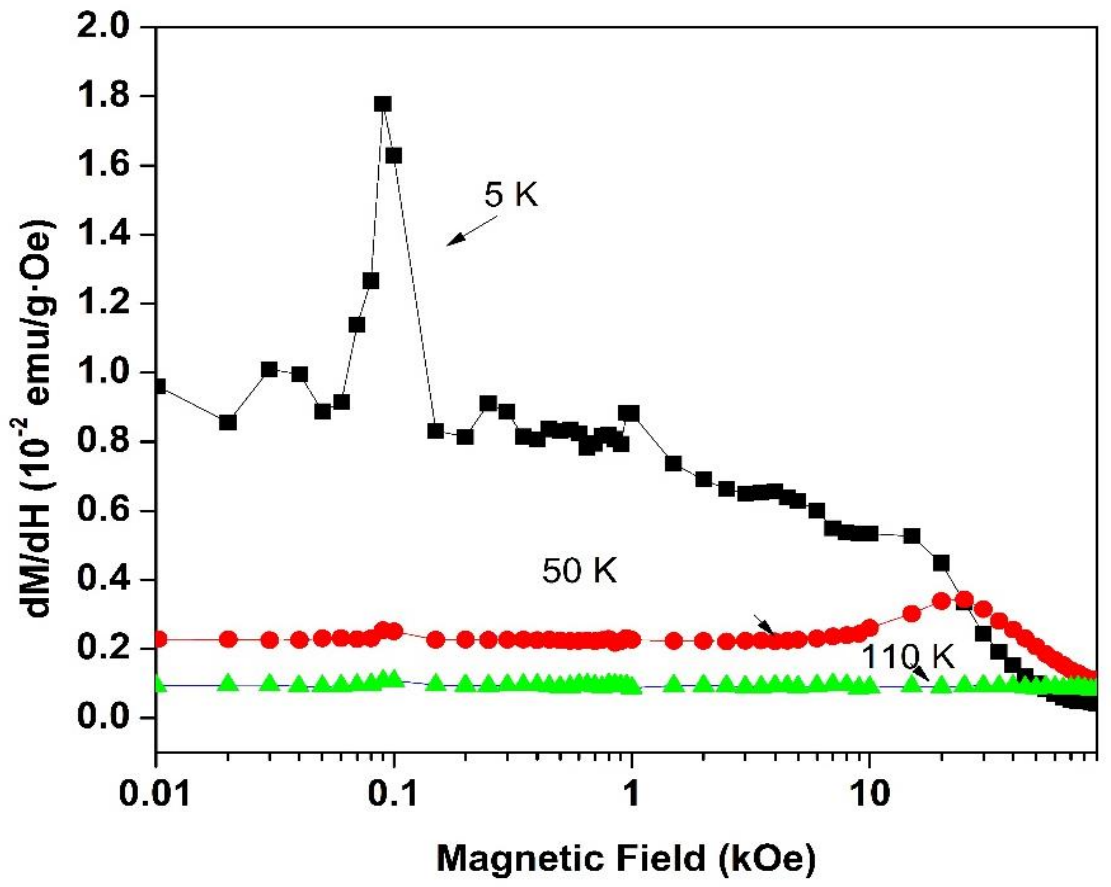

Figure 4.6: Computed dM/dH vs. $\mathrm{H}$ for the data of Fig 4.3 and Fig. 4.4 at $\mathrm{T}=5 \mathrm{~K}, 50 \mathrm{~K}$ and $110 \mathrm{~K}$. 


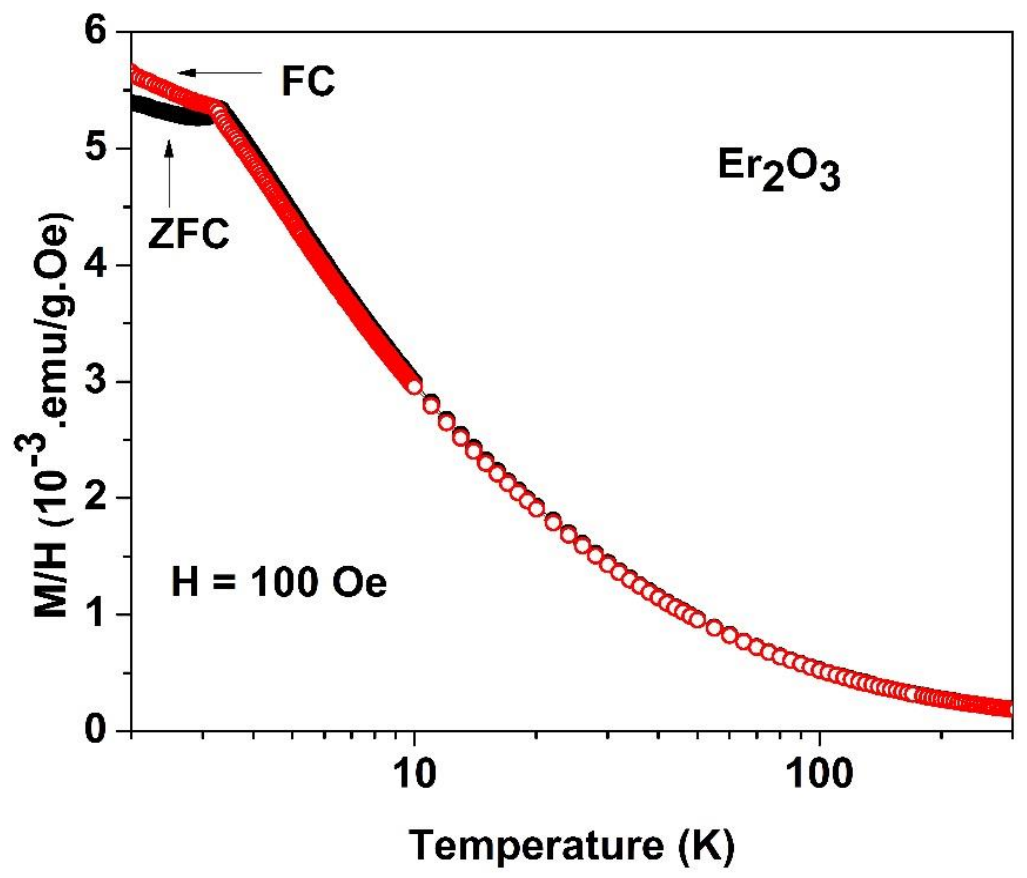

Figure 4.7: Temperature dependence $(2 \mathrm{~K}$ to $300 \mathrm{~K})$ of the magnetic susceptibility $\chi=\mathrm{M} / \mathrm{H}$ for powder sample of $\mathrm{Er}_{2} \mathrm{O}_{3}$ under $\mathrm{ZFC}$ and $\mathrm{FC}$ conditions using $\mathrm{H}=100 \mathrm{Oe}$.

\subsubsection{Magnetic Measurements on $\mathrm{Er}_{2} \mathrm{O}_{3}$ :}

The temperature dependence of the magnetic susceptibility $\mathrm{M} / \mathrm{H}$ for the $\mathrm{Er}_{2} \mathrm{O}_{3}$ powder for both the $\mathrm{ZFC}$ and FC cases in applied $\mathrm{H}=100 \mathrm{Oe}$ is shown in Figure 4.7. This temperature variations shows a peak at the known Neel temperature $\mathrm{T}_{\mathrm{N}}=3.3 \mathrm{~K}[54-56]$ below which $\chi(\mathrm{FC})>\chi(\mathrm{ZFC})$. Although a detailed discussion of the nature of magnetic ordering in $\mathrm{Er}_{2} \mathrm{O}_{3}$ is presented in next chapter, the main point to note here is that this temperature dependence is quite different from that of Er metal presented earlier and that of AlN: Er film presented below, which makes it possible to use magnetic studies to distinguish between these three cases.

\subsubsection{Magnetic Measurements on AIN and AIN:Er Films:}

The temperature dependence of the magnetic susceptibility $\chi=\mathrm{M} / \mathrm{H}$ for the films of AlN and $\mathrm{AlN}$ :Er is shown in Fig. 4.8. Measurements for both AlN and AlN:Er films are carried out at $\mathrm{H}=1$ kOe. Whereas $\chi$ of AlN is very small and nearly temperature-independent, the $\chi$ vs. T data for AlN:Er shows a Curie-like variation with $\mathrm{M}$ increasing with decreasing $\mathrm{T}$. Since the data for the ZFC and FC cases was identical in these cases, only the data for the ZFC cases is shown in Fig. 4.8. The higher $\chi$ value in the AlN:Er film vs. $\chi$ for the AlN film is associated with the doped Er. The difference susceptibility, $\chi(\mathrm{AlN}: \mathrm{Er})$ minus $\chi(\mathrm{AlN})$, is analyzed in terms of the Curie-Weiss variation $: \chi=\mathrm{C} /(\mathrm{T}$ - 
$\theta$ ). For the further analysis the Curie constant is modified from Eq. (4.4) to $\mathrm{C}=\mathrm{xN} \mu^{2} / 3 \mathrm{k}_{\mathrm{B}}$ with $\mathrm{N}$ being the number of magnetic ions/atoms per gram and $\mathrm{x}$ being the atomic $\%$ of Er with magnetic moment $\mu$ per Er and $\mathrm{k}_{\mathrm{B}}$ is the Boltzmann constant. For the data shown in Fig. 4.9 (M vs. H, T=2K) the best fit is obtained using $\mathrm{x}=1.08 \%$. This value of $\mathrm{x}$ is consistent with $\mathrm{x}=0.94 \pm 0.20 \%$ determined using XPS considering the experimental uncertainty. Therefore analysis of the magnetic data in Fig. 4.8 and later in Fig. 4.9, is carried out using $\mathrm{x}=1.08 \%$. A plot of $1 / \chi$ vs. $\mathrm{T}$ (not shown here) yielded a straight line with the intercept $\theta=-0.1 \mathrm{~K}$ to be practically zero and the slope yielding $\mathrm{C}=3.81 \times 10^{-3} \mathrm{emu}-\mathrm{K} / \mathrm{g}-\mathrm{Oe}$. The solid line through the data points in the plot of $\chi$ vs. T in Fig. 4.8 is this fit with the magnitude of $\mathrm{C}$ yielding $\mu / \mathrm{Er}=7.777 \mu_{\mathrm{B}}$ for the AlN:Er film. This magnitude of $\mu / \mathrm{Er}$ is to be compared with the reported $\mu=9$. 58 $\mu_{\mathrm{B}}$ expected for free $\operatorname{Er}$ [57] and $\mu / \mathrm{Er}^{3+}=6.68 \mu_{\mathrm{B}}$ reported in $\mathrm{Er}_{2} \mathrm{O}_{3}$ for $\mathrm{T}>\mathrm{T}_{\mathrm{N}}$ [56]. These results for AlN:Er films are consistent with $\mathrm{Er}^{3+}$ substituting for $\mathrm{Al}^{3+}$ in $\mathrm{AlN}$ : $\mathrm{Er}$. As discussed in Section 4.2.3 the parameter $\theta=\gamma \mathrm{C}$ corresponds to the magnitude of magnetic interactions. In the measurements for AlN:Er, magnitude of $\theta$ being essentially zero signifies the absence of any significant magnetic interaction among the $\mathrm{Er}^{3+}$ ions in AlN:Er films. The absence of magnetic interactions is attributed to low concentrations of the $\mathrm{Er}^{3+}$ ions in the films. Comparing the magnitudes and temperature dependences of the data of $\chi$ vs. T for elemental $\mathrm{Er}$ (Fig. 4.1), $\mathrm{Er}_{2} \mathrm{O}_{3}$ (Fig. 4.7) and AlN:Er (Fig.4.8), it is concluded that $\mathrm{Er}$ in $\mathrm{AlN}$ : Er is not present either as elemental $\mathrm{Er}$ or as $\mathrm{Er}_{2} \mathrm{O}_{3}$. In addition, the Curie law variation of $\chi$ in the thin film of AlN:Er with magnetic moment $\mu=7.777 \mu_{\mathrm{B}}$ expected for $\mathrm{Er}^{3+}$ ions confirms that the electronic state of $\mathrm{Er}$ in $\mathrm{AlN}: \mathrm{Er}$ is $\mathrm{Er}^{3+}$ resulting from the substitution of $\mathrm{Er}^{3+}$ for $\mathrm{Al}^{3+}$. The ionic size of $\mathrm{Er}^{3+}$ ions is $1.03 \AA$ and it is larger than the ionic size $0.675 \AA$ for $\mathrm{Al}^{3+}$ ions. Therefore substitution of $\mathrm{Er}^{3+}$ ion for $\mathrm{Al}^{3+}$ ion is expected to expand the lattice. The XRD results presented in Chapter 2 (Fig. 2.5) are consistent with this observation.

Yang et al. [57] carried out electron paramagnetic resonance (EPR) measurements on $\mathrm{Er}^{3+}$ ions introduced during growth of aluminum nitride single crystals prepared by the seeded sublimationrecondensation method. The findings from EPR measurements carried out at $4.5 \mathrm{~K}$ only are that $\mathrm{Er}^{3+}$ substitutes for $\mathrm{Al}^{3+}$ sitting in axial symmetry defined by the c-axis with $\mathrm{g}(\|)=4.337$ and $\mathrm{g}(\perp)=7.647$. Since the ground state has an effective spin $S=1 / 2$, using $\mu^{2}=\mu_{\mathrm{B}}{ }^{2} \mathrm{~g}^{2} \mathrm{~S}(\mathrm{~S}+1), \mu / \mathrm{Er}=7.777 \mu_{\mathrm{B}}$ determined above from the $\chi$ vs. $\mathrm{T}$ data yields $\mathrm{g}=8.980$. In this work magnetic measurements on the sputtered AlN:Er thin films were done with applied magnetic field parallel to the thin film and hence $\mathrm{H} \perp \mathrm{c}$-axis and so the measured $\mathrm{g}=8.980$ should nearly represent $\mathrm{g}(\perp)=7.647$ measured by EPR at $4.5 \mathrm{~K}$ as is the case here. The larger $g=8.980$ compared to $g(\perp)=7.647$ measured by EPR at $4.5 \mathrm{~K}$ is proposed to result from contributions from excited states as discussed in the following. 


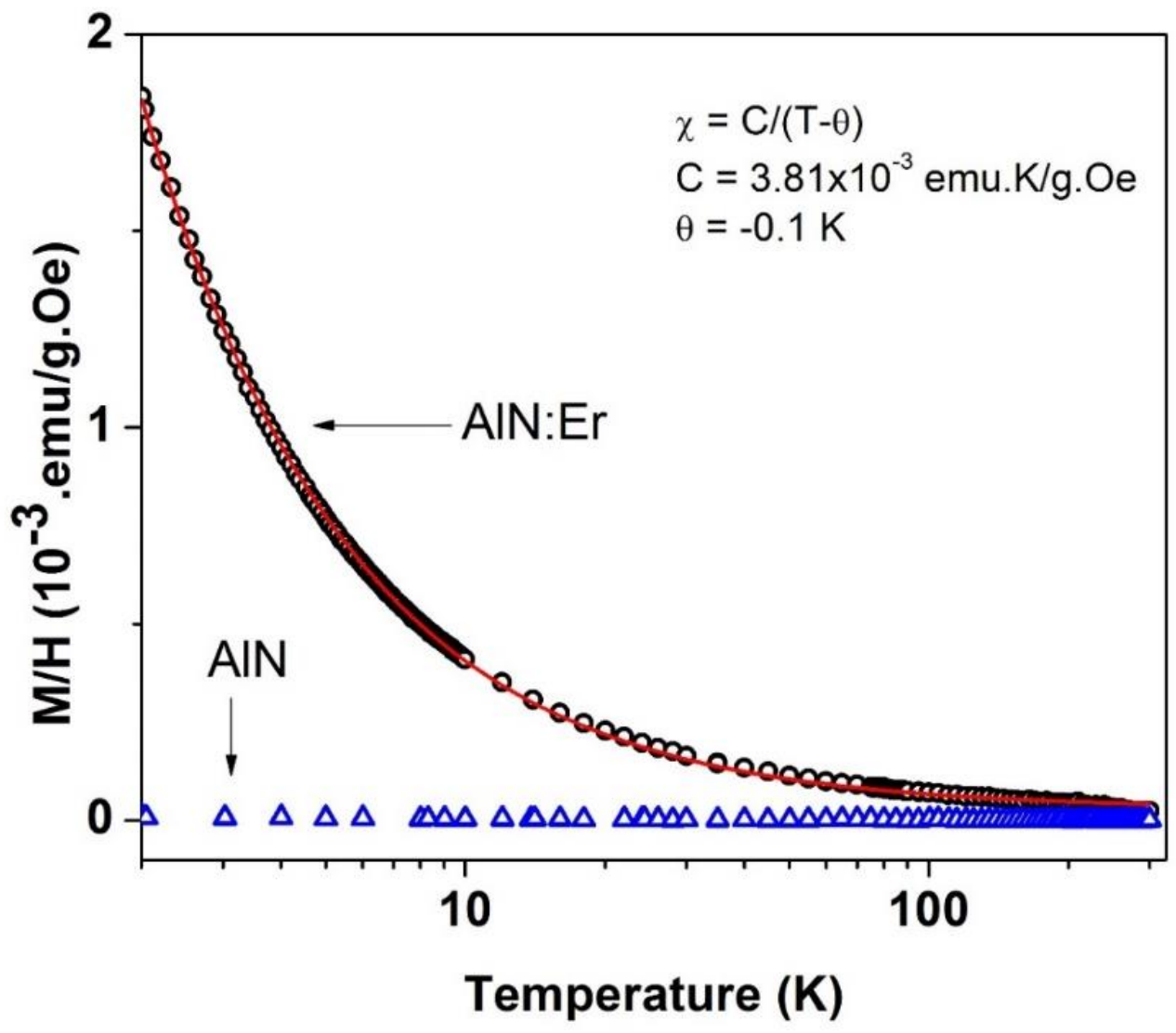

Figure 4.8: Shown are the experimental results on the temperature dependence of the magnetic susceptibility M/H for the thin films of AlN and AlN:Er with the solid line fit to the Curie-Weiss law with the parameters given in the figure.

Isothermal data of $\mathrm{M}$ vs. $\mathrm{H}$ at low temperature can be used to obtain more information on the electronic state of a magnetic ion (such as $\mathrm{Er}^{3+}$ ) in a diamagnetic host [58]. For the AlN:Er film $\mathrm{M}$ vs. $\mathrm{H}$ measurements were carried out for $\mathrm{H}$ up to $90 \mathrm{kOe}$ and at $\mathrm{T}=2 \mathrm{~K}$ and data is shown in Fig. 4.9. In the presence of axial field of AlN, the lowest lying ${ }^{4} \mathrm{I}_{15 / 2}$ electronic state of free $\mathrm{Er}^{3+}$ is split into $8 \mathrm{Kramer}$ doublets $[57,59]$. The other excited states of $\mathrm{Er}^{3+}$ are ${ }^{4} \mathrm{I}_{13 / 2},{ }^{4} \mathrm{I}_{11 / 2}$ and ${ }^{4} \mathrm{I}_{9 / 2}$ each of which split into 7, 6 and 5 doublets respectively in the axial field. Fluorescence studies of AlN:Er have shown [59] that energy splitting $E_{n}(n=0,1,2, \ldots ., 7)$ of the 8 Kramer doublets of ${ }^{4} I_{15 / 2}$ in units of $\mathrm{cm}^{-1}$ are: $0,7,31$, 98, 135, 190, 239 and $275 \mathrm{~cm}^{-1}$ respectively. The energy levels of the other excited states are shown in Table 4.1 (from Ref. [59]). In a magnetic field $\mathrm{H}$, each of the 8 Kramer doublets will split up into two Zeeman levels each with energies equal to $E_{n}+\gamma H$ and $E_{n}-\gamma H$ with $\gamma=g \mu_{B} / 2$. At the higher temperatures where the Curie-Weiss law is valid and at $2 \mathrm{~K}$ in $\mathrm{H}$ up to $90 \mathrm{kOe}$ used here, some of the 
energy levels other than the ground state will also be populated. Therefore, these levels will also contribute to $\mathrm{M}$. In this case, magnetization $\mathrm{M}$ of a multilevel system can be calculated using the Eq. (4.7) modified by the concentration $\mathrm{x}$ of $\mathrm{Er}^{3+}$ in $\mathrm{AlN}$ :

$$
M=-N_{A} x \frac{\sum_{n} \frac{\partial E_{n}}{\partial H} e^{-\beta E_{n}}}{\sum_{n} e^{-\beta E_{n}}}
$$

Here $E_{n}$ is the energy of the nth level in a magnetic field $H, \beta=1 / k_{\beta} T, N_{A}=$ Avogadro's number and $x$ is the concentration of the magnetic ions. From Eq. (4.20), it follows that if the g-values for all the levels is the same ( $\gamma=\gamma_{1}$ in Fig. 4.9), then:

$$
M=M_{0} \tanh \left(\beta g \mu_{B} H / 2\right)
$$

with $\mathrm{M}_{0}=\left(\mathrm{N}_{\mathrm{A}} x \mathrm{~g} \mu_{\mathrm{B}} / 2\right)$. Using $\mathrm{g}(\perp)=7.647$ from $\mathrm{EPR}, \mathrm{x}=0.0108$ and molecular weight $=21.45$ noted earlier for the thin film of AlN:Er yields $M_{0}=10.75 \mathrm{emu} / \mathrm{g}$. In Fig. 4.9, the lower solid line for $\gamma=\gamma_{1}$ represents this case. In this case the saturation of $\mathrm{M}$ above $20 \mathrm{kOe}$ predicted by Eq. (4.21) is not observed in Fig. 4.9 but $\mathrm{M}$ is still increasing with increase in $\mathrm{H}$ up to the maximum $\mathrm{H}=90 \mathrm{kOe}$

\begin{tabular}{|c|c|c|c|c|c|}
\hline Manifold & $\begin{array}{l}\text { Energy } \\
\left(\mathrm{cm}^{-1}\right)\end{array}$ & $\begin{array}{c}\text { Energy } \\
(\mathrm{meV})\end{array}$ & Manifold & $\begin{array}{l}\text { Energy } \\
\left(\mathrm{cm}^{-1}\right)\end{array}$ & $\begin{array}{l}\text { Energy } \\
(\mathrm{meV})\end{array}$ \\
\hline \multirow{9}{*}{${ }^{4} \mathrm{I}_{15 / 2}$} & 0 & 0 & ${ }^{4} I_{11 / 2}$ & 10108 & 1253.3 \\
\hline & 7 & 0.8 & & 10193 & 1263.9 \\
\hline & 31 & 3.8 & & 10213 & 1266.3 \\
\hline & 98 & 12.2 & & - & - \\
\hline & 135 & 16.7 & & - & - \\
\hline & 190 & 23.6 & & - & - \\
\hline & 239 & 29.6 & & & \\
\hline & 275 & 34.1 & ${ }^{4} I_{9 / 2}$ & 12,238 & 1517.4 \\
\hline & & & & 12,283 & 1523.0 \\
\hline \multirow[t]{7}{*}{${ }^{4} \mathrm{I}_{13 / 2}$} & 6515 & 807.8 & & 12,315 & 1527.0 \\
\hline & 6527 & 809.3 & & 12,407 & 1538.4 \\
\hline & 6538 & 810.7 & & 12,419 & 1539.9 \\
\hline & 6553 & 812.5 & & & \\
\hline & 6608 & 819.3 & & & \\
\hline & 6632 & 822.3 & & & \\
\hline & 6680 & 828.3 & & & \\
\hline
\end{tabular}
available in the PPMS magnetometer.

Table 4.1: Energy levels of $\mathrm{Er}^{3+}$ from Ref. [59].

The above discrepancy can be resolved if the g-values of the higher Kramer doublets are different from that of the ground state [60]. Since at $2 \mathrm{~K}$, only the $\mathrm{E}_{0}$ and $\mathrm{E}_{1}$ levels will be appreciably 
populated, $\gamma_{1}=g_{1} \mu_{\mathrm{B}} / 2$ is used for the $\mathrm{E}_{1}$ level, different from $\gamma=\mathrm{g} \mu_{\mathrm{B}} / 2(\mathrm{~g}=7.647)$ for the $\mathrm{E}_{0}$ level. Substituting these values in Eq. (4.20) yields

$$
M=M_{0} \frac{\sinh (\beta \gamma H)+\left(\gamma_{1} / \gamma\right) \sinh \left(\beta \gamma_{1} H\right) e^{-\beta E_{1}}}{\cosh (\beta \gamma H)+\cosh \left(\beta \gamma_{1} H\right) e^{-\beta E_{1}}}
$$

It is straightforward to show that for $\gamma=\gamma_{1}$, Eq. (4.22) reduces to Eq. (4.21). In this analysis, the ratio $\left(\gamma_{1 /} \gamma\right)$ is varied to get the best fit with the data in Fig. 4.9 yielding $\gamma_{1 /} \gamma=1.207$ corresponding to $g_{1}=$ 9.230. From the Curie- Weiss fit of the data up to $300 \mathrm{~K}, \mathrm{~g}=8.980$ was obtained using $\mu=7.7772 \mu_{\mathrm{B}}$. At these higher temperatures, the levels $\mathrm{E}_{2}, \mathrm{E}_{3}$ etc are also expected to be populated. Therefore, $\mathrm{g}=$ 8.980 represents the effective g-values of all these levels. Thus contributions from the higher Kramer doublets are important when data at higher temperatures or higher magnetic fields are involved, unlike the case of EPR studies at low temperatures and low magnetic fields.

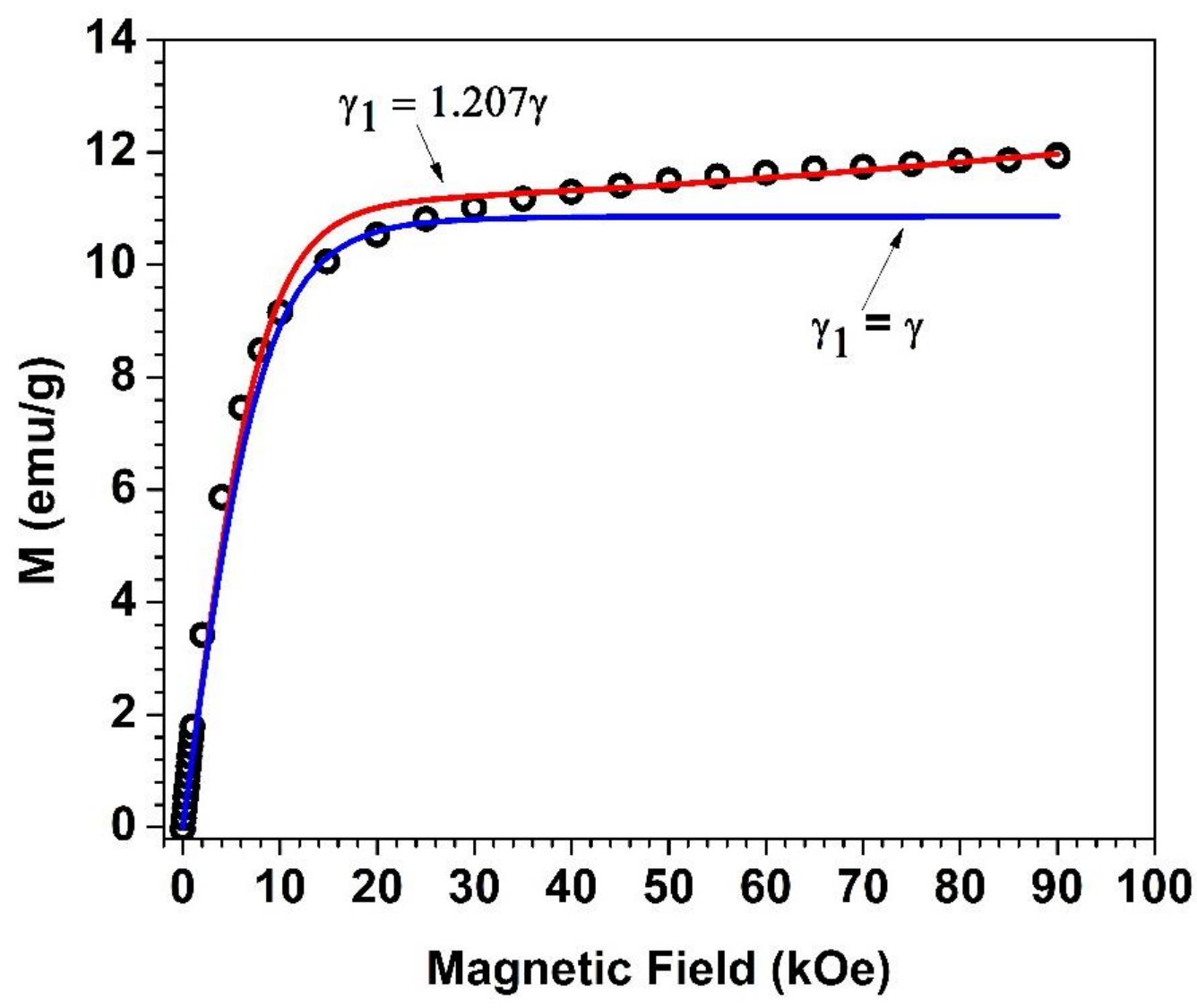

Figure 4.9: $\mathrm{M}$ vs $\mathrm{H}$ data (circles) for the AlN:Er thin film measured at $2 \mathrm{~K}$. Red line corresponds to fit to Eq. 3 with $\gamma_{1}=1.207 \gamma$ and blue line corresponds to $\gamma_{1}=\gamma$. 


\subsection{Summary and Conclusions:}

In this Chapter, analysis of the results on the temperature and magnetic field variations of the magnetization in a sputtered film of AlN:Er have been presented. This analysis has established $\mathrm{Er}^{3+}$ as the electronic state of Er in AlN:Er. The oxygen present in the thin film is present as Al-O in a thin layer on the surface of the film and not as $\mathrm{Er}_{2} \mathrm{O}_{3}$. These results have established that $\mathrm{Er}^{3+}$ substitutes for $\mathrm{Al}^{3+}$ during sputtering and preferentially aligns the film with c-axis normal to the film. The improved performance in the piezoelectric properties of AlN:Er films reported earlier in Chapter III likely results from this preferential alignment. Equally important are the results presented here showing the usefulness of magnetic measurements for determining the electronic states of magnetic additives in

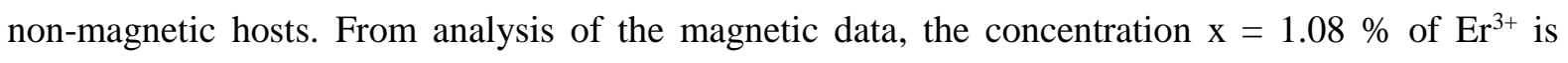
determined which is in good agreement with $\mathrm{x}=0.94 \pm 0.20 \%$ determined using the analysis of the XPS data described in Chapter 2. 


\section{CHAPTER 5 - Magnetic Properties of $\mathrm{Er}_{2} \mathrm{O}_{3}$}

\subsection{Introduction:}

In this chapter, magnetic properties of two samples of powder $\mathrm{Er}_{2} \mathrm{O}_{3}$ are presented. The structural characterization of these samples and the procedures for magnetic measurements were presented in Chapter 2. Since $\mathrm{Er}_{2} \mathrm{O}_{3}$ was one of the possibilities for the electronic form of $\mathrm{Er}$ in the AlN:Er films, it was considered necessary to understand the magnetic properties of $\operatorname{Er}_{2} \mathrm{O}_{3}$.Most of the results presented in this chapter have appeared in a recent publication by the author [56].

\subsection{Previous Studies on the Magnetic Properties of $\mathrm{Er}_{2} \mathrm{O}_{3}$ :}

The initial studies of the magnetic properties of rare-earth (RE) oxides $\mathrm{Dy}_{2} \mathrm{O}_{3}, \mathrm{Yb}_{2} \mathrm{O}_{3}$, and $\mathrm{Er}_{2} \mathrm{O}_{3}$ showed that the magnetic ordering in these oxides occurred only below $4 \mathrm{~K}[54,55,61]$. Magnetic studies by Bonrath et al. [54] carried out from $1.1 \mathrm{~K}$ to $4.2 \mathrm{~K}$ in magnetic field $\mathrm{H}=3.5 \mathrm{kOe}$ showed antiferromagnetic (AFM) ordering in these oxides with the $\mathrm{N}^{\prime}$ eel temperature $\mathrm{T}_{N}=1.2 \mathrm{~K}$ for $\mathrm{Dy}_{2} \mathrm{O}_{3}, \mathrm{~T}_{N}$ $=2.3 \mathrm{~K}$ for $\mathrm{Yb}_{2} \mathrm{O}_{3}$ and $\mathrm{T}_{N}=3.4 \mathrm{~K}$ for $\mathrm{Er}_{2} \mathrm{O}_{3}$. This was followed by the studies of Moon et al. [55] who used neutron diffracting investigations to determine the nature of magnetic ordering in $\mathrm{Yb}_{2} \mathrm{O}_{3}$ and $\mathrm{Er}_{2} \mathrm{O}_{3}$ following some calculations on this ordering by Bertaut et al. [61]. These studies showed that the magnetic ordering in these systems is quite complex in that there is no single unique easy direction of ordering for the $32 \mathrm{RE}$ ions in the unit cell. These investigations led to the conclusion that neither the dipole-dipole interaction nor nearest-neighbor super-exchange interaction can explain the observed magnetic ordering. Low temperature calorimetric investigations of $\mathrm{Er}_{2} \mathrm{O}_{3}$ combined with magnetic susceptibility vs. temperature studies below $100 \mathrm{~K}$ were reported by Tang et al. [62]. These studies reported $\mathrm{T}_{N}=3.3 \mathrm{~K}$ and suggested the possibility that magnetic ordering may be due to exchange coupling among the $\mathrm{Er}^{3+}$ on the $\mathrm{C}_{2}$ sites only although they did not refer to the above noted studies by Moon et al. [55], analysis by Bertaut et al. [61], and magnetic studies of Bonrath et al. [54]. Dean and Bloor [63] have reported far-infrared and optical spectroscopic investigations of the electronic excitations of $\mathrm{Er}^{3+}$ in $\mathrm{Er}_{2} \mathrm{O}_{3}$ [63].

A reason for the complicated magnetic ordering in these systems is the comparatively large

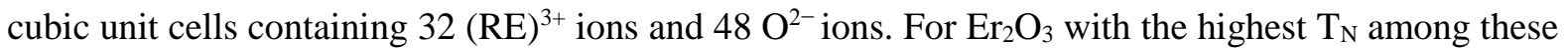
oxides, the lattice constant $\mathrm{a}=10.55 \AA$ in the bixbyite structure [55]. The atomic model of $\mathrm{Er}_{2} \mathrm{O}_{3}$ structure is shown in Fig. 5.1 and the directions of ordering of the magnetic moments in the unit cell of $\mathrm{Er}_{2} \mathrm{O}_{3}$ (shown in Fig. 5.2) as determined by neutron diffraction studies [55] show no unique ordering direction. Instead, these studies showed that of the $32 \mathrm{Er}^{3+}$ ions in the unit cell, 24 occupy sites of $\mathrm{C}_{2}$ 
symmetry (see Fig. 5.1) with $\mu=5.36 \mu_{\mathrm{B}}$ aligned antiferromagnetically along the three mutually orthogonal $<100\rangle$ axes and the remaining 8 occupy sites of $\mathrm{C}_{3 \mathrm{i}}$ symmetry with $\langle 111\rangle$ as the symmetry axis (see Fig. 5.1) and $\mu=6.06 \mu_{\mathrm{B}}$. Bertaut et al. reported $\mu=5.7 \mu_{\mathrm{B}}$ for both sites [61]. It is evident from Fig. 5.2 that that there is no single unique direction of magnetic ordering. Therefore $\mathrm{Er}_{2} \mathrm{O}_{3}$ can be termed as a non-collinear antiferromagnet.
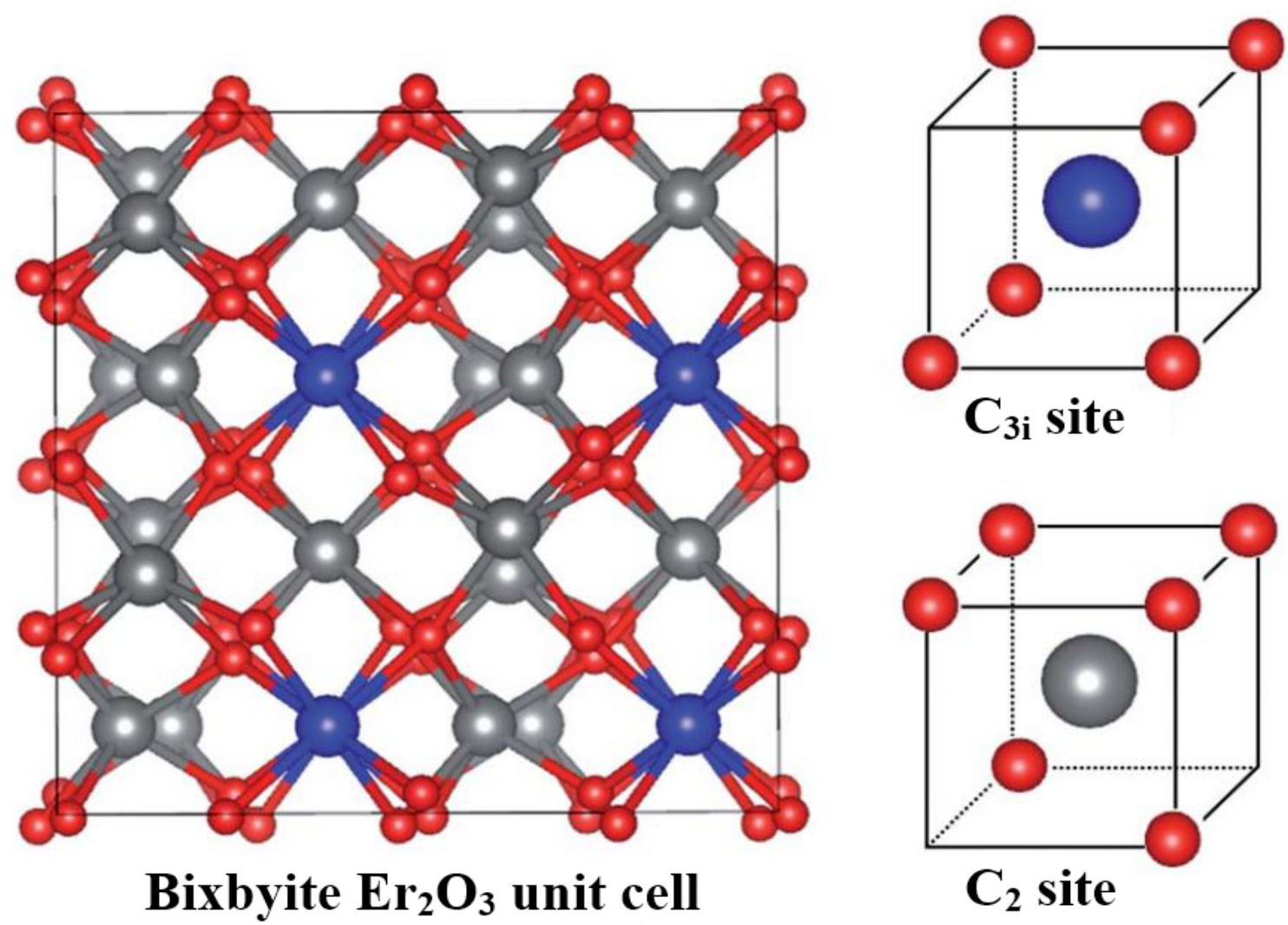

Figure 5.1: Atomic model of bixbyite structure of $\mathrm{Er}_{2} \mathrm{O}_{3}$ (Modified from Ref. [64]). Red spheres are O atoms, blue and grey spheres are $\mathrm{Er}$ atoms at $\mathrm{C}_{3 \mathrm{i}}$ and $\mathrm{C}_{2}$ sites respectively.

\subsection{Experimental Results:}

Measurements of magnetization $\mathrm{M}$ versus temperature $\mathrm{T}$ and applied field $\mathrm{H}$ were done using a vibrating sample magnetometer of the commercial PPMS magnetometer from Quantum Design Inc. For the zero-field (ZFC) case, samples were cooled in magnetic field $\mathrm{H}=0$ Oe to $2 \mathrm{~K}$, the lowest temperature attainable with the PPMS, magnetic field $\mathrm{H}=100$ Oe was then applied and data taken with increasing temperatures after stabilization of the temperature at each point. For the field-cooled (FC) case, the samples were cooled from $300 \mathrm{~K}$ to $2 \mathrm{~K}$ in $\mathrm{H}=100$ Oe followed by data acquisition with increasing $\mathrm{T}$ in a similar manner. 


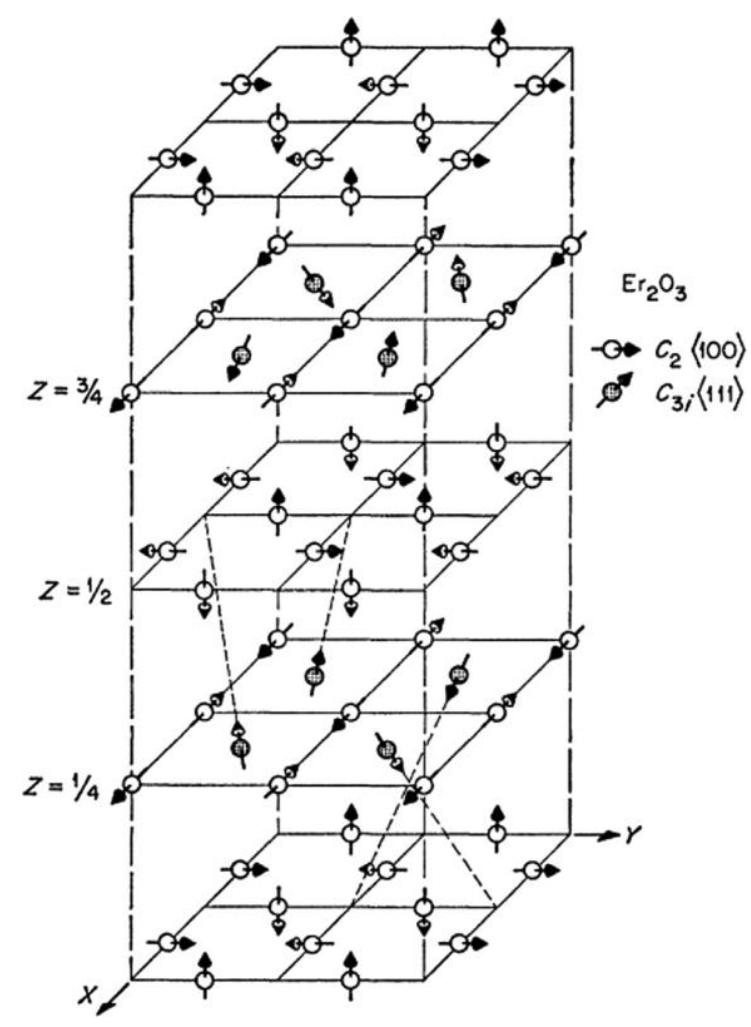

Figure 5.2: Magnetic ordering of $\mathrm{Er}^{3+}$ ions in the cubic unit cell of $\mathrm{Er}_{2} \mathrm{O}_{3}$ (reproduced from Moon et al. [55]) The unit cell has been expanded along the z-direction for clarity and the positions of the oxygen ions situated between the $\mathrm{Er}^{3+}$ ions are not shown.

In Fig. 5.3 are shown the plots of the magnetic susceptibility $\chi=(\mathrm{M} / \mathrm{H})$ vs. T for the two samples measured with $\mathrm{H}=100 \mathrm{Oe}$. The inset of the figure shows the expanded views of the data below $6 \mathrm{~K}$. It is evident that there is a peak near $3.3 \mathrm{~K}$ in the $\chi(\mathrm{ZFC})$ vs. $\mathrm{T}$ data of both samples; in addition there is a bifurcation of the FC data from that of the ZFC data below this peak. This peak is associated with the $\mathrm{N}^{\prime}$ eel temperature $\mathrm{T}_{\mathrm{N}}$ of $\mathrm{AFM}$ ordering with more accurate determination of $\mathrm{T}_{\mathrm{N}}$ given in section 5.4 below. Comparing the data for the two samples in Fig. 5.3, the magnitudes of $\chi$ for $n-\mathrm{Er}_{2} \mathrm{O}_{3}$ at a given temperature are larger than those for the $\mathrm{b}-\mathrm{Er}_{2} \mathrm{O}_{3}$ sample. In Chapter 2, XRD measurements on both samples of $\mathrm{Er}_{2} \mathrm{O}_{3}$ sample were presented which showed the presence of minute quantities of nonmagnetic $\mathrm{SiO}_{2}$ impurities in $\mathrm{b}-\mathrm{Er}_{2} \mathrm{O}_{3}$. The lower magnitudes of magnetic susceptibility in $\mathrm{b}-\mathrm{Er}_{2} \mathrm{O}_{3}$ relative to those in $n-\mathrm{Er}_{2} \mathrm{O}_{3}$ is explained in terms of the presence of non-magnetic $\mathrm{SiO}_{2}$ whose effect is to lower the concentration of $\mathrm{Er}_{2} \mathrm{O}_{3}$ in $\mathrm{b}-\mathrm{Er}_{2} \mathrm{O}_{3}$. In addition, it is believed that the results reported here on the $\mathrm{n}-\mathrm{Er}_{2} \mathrm{O}_{3}$ sample are not influenced by finite-size effects since such effects on the magnetic 
properties of the nanoparticles become noticeable only for particles of size less than about $30 \mathrm{~nm}[65$, $66]$.

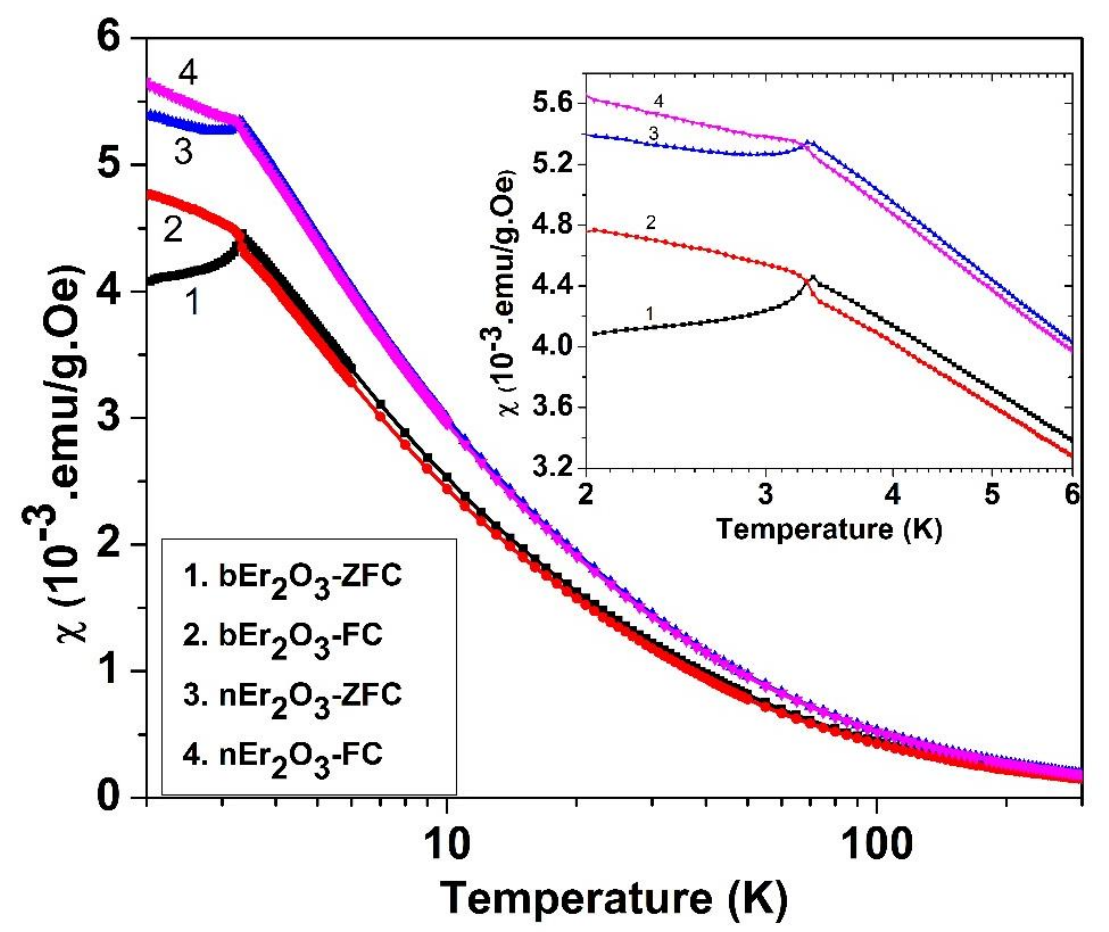

Figure 5.3: Temperature dependence of the magnetic susceptibility of the two samples of $\mathrm{Er}_{2} \mathrm{O}_{3}$ for the zero-field-cooled and field-cooled cases in $\mathrm{H}=100 \mathrm{Oe}$. The inset shows the expanded view of the data for $\mathrm{T}<6 \mathrm{~K}$.

For both samples, the magnetic field dependence of the magnetization $\mathrm{M}$ measured at $2 \mathrm{~K}$ for $\mathrm{H}$ up to $90 \mathrm{kOe}$ is shown in Fig. 5.4. Noting that $\mathrm{M}$ for $\mathrm{n}-\mathrm{Er}_{2} \mathrm{O}_{3}$ is greater than that for $\mathrm{b}-\mathrm{Er}_{2} \mathrm{O}_{3}$ as explained above, these variations of $\mathrm{M}$ vs. $\mathrm{H}$ for the two samples are near identical with a change in the slope of the variations occurring near $30 \mathrm{kOe}$. In addition, hysteresis is absent for both samples as shown in the inset of Fig. 5.4, confirming the presence of antiferromagnetic ordering. The saturation magnetization was estimated from the plot of $\mathrm{M}$ vs. $1 / \mathrm{H}$ extrapolating to $1 / \mathrm{H}$ going to zero (Fig. 5.5).

In order to further determine the nature of magnetism in $\mathrm{Er}_{2} \mathrm{O}_{3}, \mathrm{M}$ vs. $\mathrm{H}$ variations were carried out at several temperatures with increase in temperature through $\mathrm{T}_{\mathrm{N}}$. These measurements were done at $0.1 \mathrm{~K}$ intervals between $2 \mathrm{~K}$ and $4 \mathrm{~K}$ for $\mathrm{n}-\mathrm{Er}_{2} \mathrm{O}_{3}$ (see Fig. 5.6). It is evident that the $\mathrm{M}$ vs. $\mathrm{H}$ variation in the $2 \mathrm{~K}$ to $4 \mathrm{~K}$ temperature range are qualitatively similar. For the $2 \mathrm{~K}$ to $10 \mathrm{~K}$ range, changes in the temperature dependence of the magnetic susceptibility $\chi(Z F C)=M / H$ for the several fixed values of $\mathrm{H}$ up to $\mathrm{H}=20 \mathrm{kOe}$ are shown in Fig. 5.7 From these plots, it can be inferred that the peak position of $\chi$ approximately representing $\mathrm{T}_{\mathrm{N}}$ shifts to the lower temperatures with increase in $\mathrm{H}$ so that for $\mathrm{H}=$ 
$20 \mathrm{kOe}$, the Neel temperature $\mathrm{T}_{\mathrm{N}}$ if present, is definitely below $2 \mathrm{~K}$. Further discussion on these results is given later.

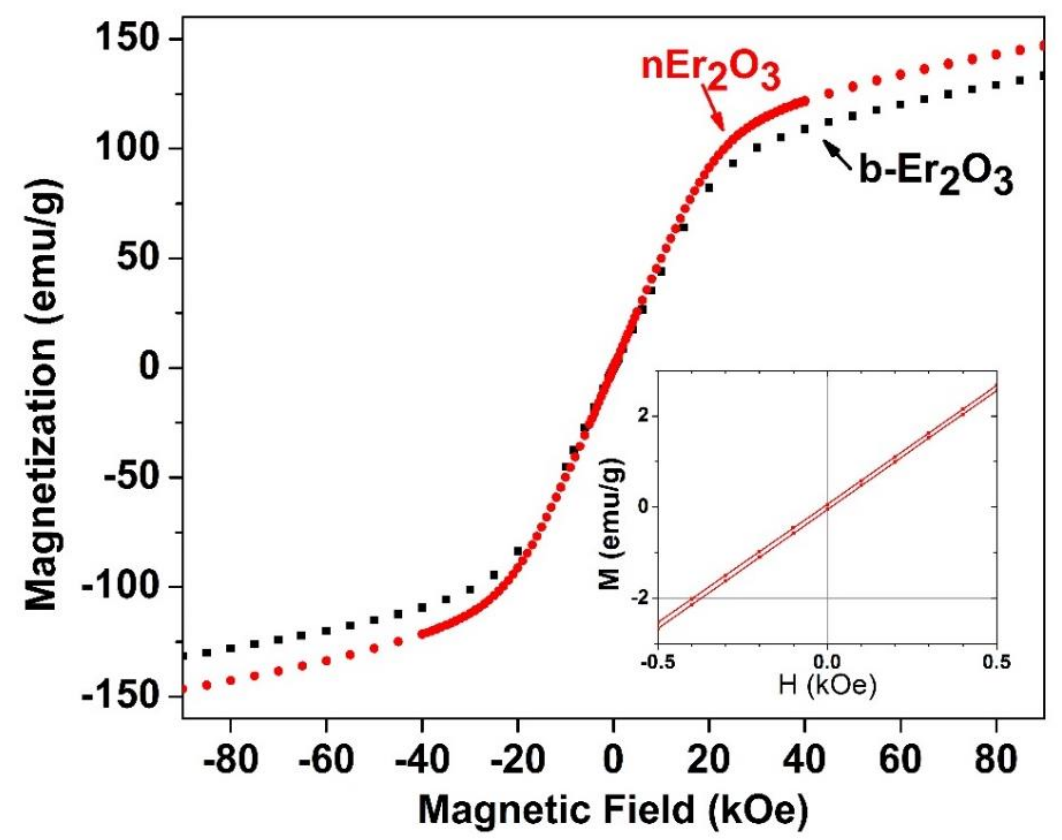

Figure 5.4: Magnetic field dependence of the magnetization of the $b-\mathrm{Er}_{2} \mathrm{O}_{3}$ and $n-\mathrm{Er}_{2} \mathrm{O}_{3}$ samples at 2 K. Observed hysteresis of few Oe (inset) is within the experimental uncertainty of setting the field.

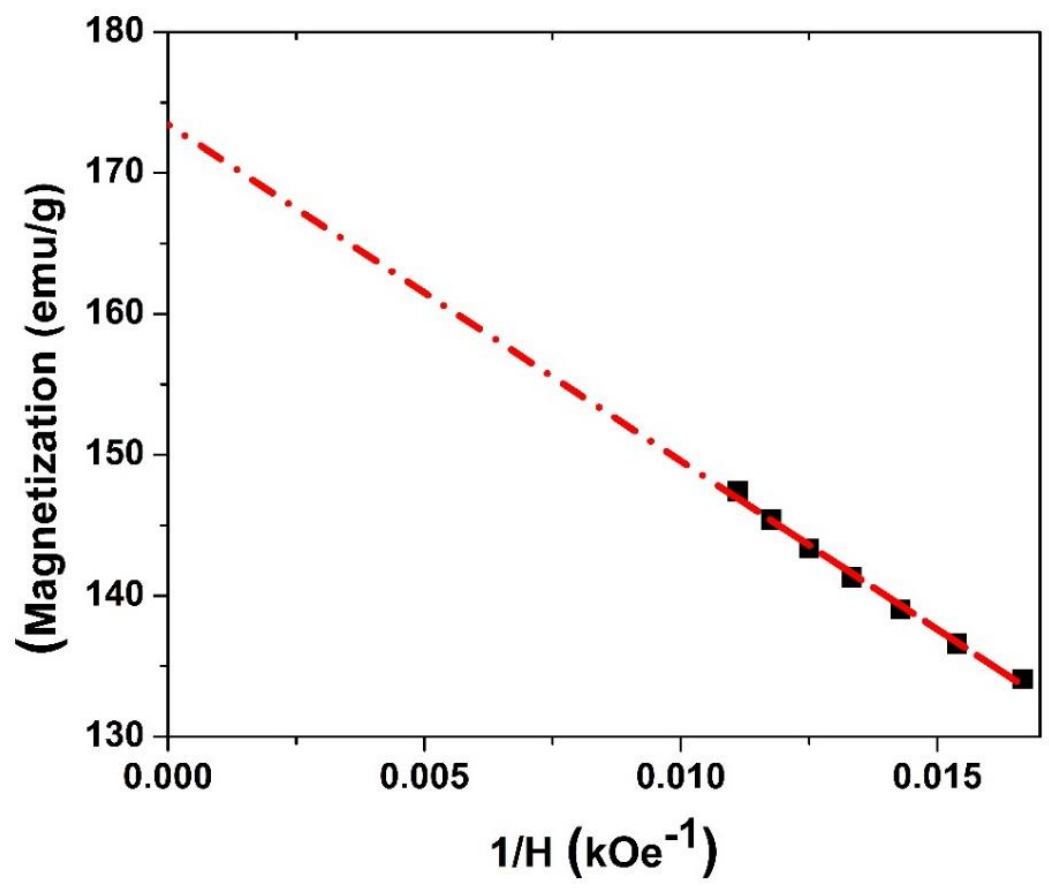

Figure 5.5: The plots of $\mathrm{M}$ vs. $1 / \mathrm{H}$ to estimate the saturation magnetization $\mathrm{M}_{\mathrm{S}}$ in the limit of $1 / \mathrm{H}=0$. 


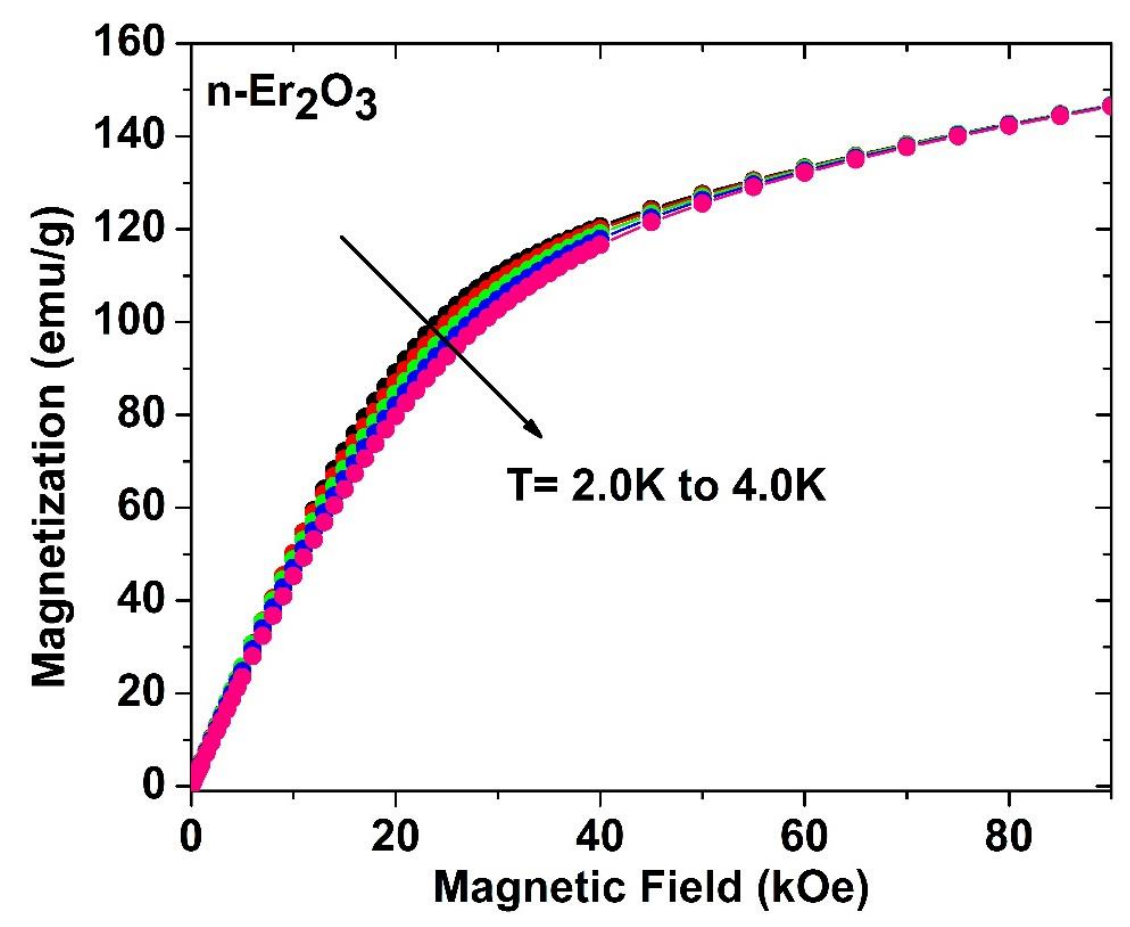

Figure 5.6: Magnetic field dependence of the magnetization at several temperatures for $n-\mathrm{Er}_{2} \mathrm{O}_{3}$. For clarity, data for only a few select temperatures are shown.

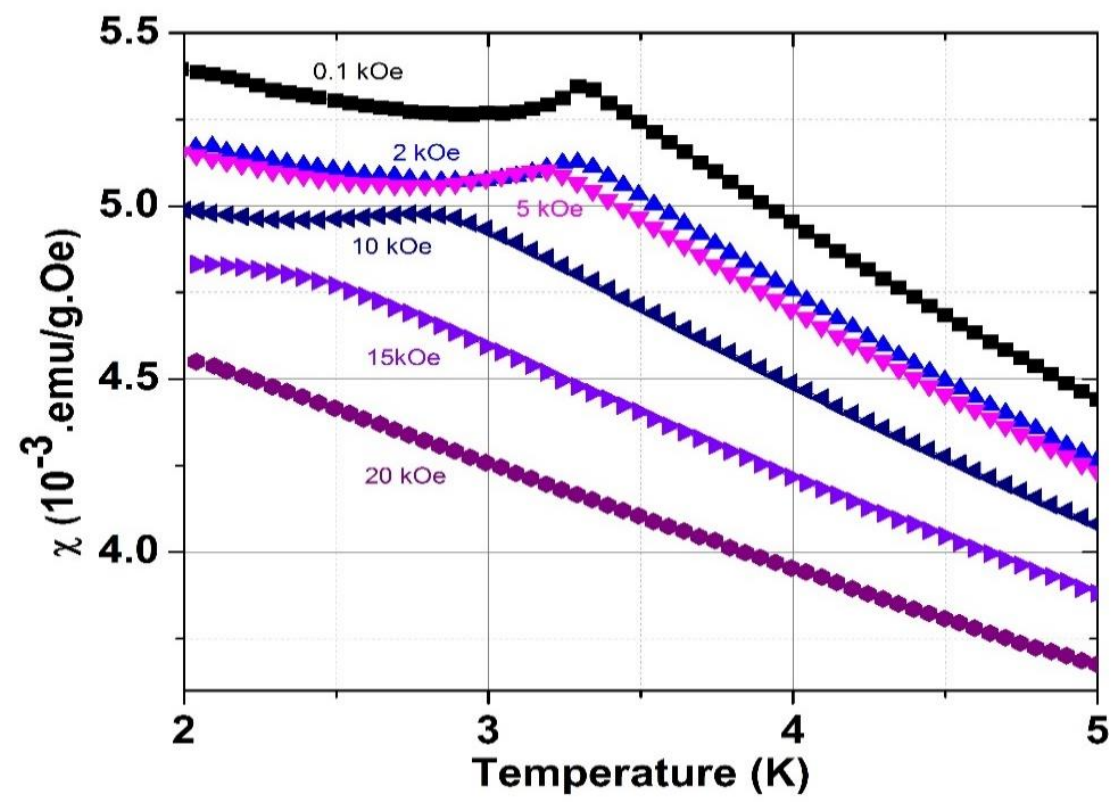

Figure 5.7: Temperature dependence ( $2 \mathrm{~K}$ to $5 \mathrm{~K}$ ) of the magnetic susceptibility of $\mathrm{n}-\mathrm{Er}_{2} \mathrm{O}_{3}$ for the zerofield-cooled case in different $\mathrm{H}$ from 100 Oe to $20 \mathrm{kOe}$. 


\subsection{Analysis, Discussion and Interpretation of the Results:}

The data of magnetic susceptibility versus temperature shown earlier in Fig. 5.3 is analyzed in terms of the Curie-Weiss $(\mathrm{CW})$ variation $(\chi=\mathrm{C} /(\mathrm{T}-\theta))$ discussed in Chapter 4 . For this analysis, $\chi^{-1}$ vs. $\mathrm{T}$ is plotted in Fig. 5.8. It is evident that the data for both samples fit extremely well with the CW variation yielding the parameter $\theta=-9.5 \mathrm{~K}(-10.2 \mathrm{~K})$ and the Curie constant $\mathrm{C}=0.0485(0.0584)$ $\mathrm{emu} \cdot \mathrm{K} / \mathrm{g}$. Oe for $\mathrm{b}-\mathrm{Er}_{2} \mathrm{O}_{3}\left(\mathrm{n}-\mathrm{Er}_{2} \mathrm{O}_{3}\right)$ sample. We use $\mathrm{C}=\mathrm{N} \mu^{2} / 3 \mathrm{k}_{\mathrm{B}}$ to determine $\mu / \mathrm{Er}^{3+}$. Here $\mathrm{N}$ is the number of magnetic ions per gram, each with magnetic moment $\mu$, and $\mathrm{k}_{\mathrm{B}}$ is the Boltzmann constant. This procedure yields the calculated $\mu / \mathrm{Er}^{3+}=6.09 \mu_{\mathrm{B}}\left(6.68 \mu_{\mathrm{B}}\right)$ for $\mathrm{b}-\mathrm{Er}_{2} \mathrm{O}_{3}\left(\mathrm{n}-\mathrm{Er}_{2} \mathrm{O}_{3}\right)$ as the average magnitude of the paramagnetic magnetic moment per $\mathrm{Er}^{3+}$ ion. For b- $\mathrm{Er}_{2} \mathrm{O}_{3}$, the lower magnitude of $\mu / \mathrm{Er}^{3+}$ is explained in terms of the presence of $\mathrm{SiO}_{2}$ impurity as discussed earlier. For this reason, in the discussions given below, only the parameters $\mu / \mathrm{Er}^{3+}=6.68 \mu_{\mathrm{B}}$ and $\theta=-10.2 \mathrm{~K}$ observed for $\mathrm{n}-\mathrm{Er}_{2} \mathrm{O}_{3}$ are used. For the limited temperature range of $\mathrm{T}<100 \mathrm{~K}$, Tang et al. [62] fitted the data of $\chi$ vs. T to the $\mathrm{CW}$ law, reported $\theta=-9.5 \mathrm{~K}$ and $\mu=7.1 \mu_{\mathrm{B}}$ for $\mathrm{Er}^{3+}$. Most likely, this somewhat larger magnitude $\mu$ reported by Tang et al. [62] is due to the limited temperature range used for fitting the CW law.

As noted earlier, Moon et al. [55] using neutron diffusion measurements reported the lower magnitudes of $\mu=5.36 \mu_{\mathrm{B}}\left(6.06 \mu_{\mathrm{B}}\right)$ for $\mathrm{Er}^{3+}$ ions on the $24 \mathrm{C}_{2}\left(8 \mathrm{C}_{3 \mathrm{i}}\right)$ sites yielding an average value of $5.54 \mu_{\mathrm{B}}$. Bertaut et al. reported an average $\mu=5.70 \mu_{\mathrm{B}}$ for both sites [61]. In order to reconcile these lower magnitudes of $\mu$ measured for $\mathrm{T}<\mathrm{T}_{\mathrm{N}}$ with $\mu=6.68 \mu_{\mathrm{B}}$ derived from the fit to $\mathrm{CW}$ law above $\mathrm{T}_{\mathrm{N}}$, the zero-point spin deviations predicted by spin-wave theory of antiferromagnets [67] and verified experimentally is invoked according to which the measured value of $\langle\mathrm{S}\rangle$ for $\mathrm{T}<<\mathrm{T}_{\mathrm{N}}$ is reduced by $15.6 \%$ for the $S=1 / 2$ system [68]. Reducing $\mu / \mathrm{Er}^{3+}=6.68 \mu_{\mathrm{B}}$ by $15.6 \%$ yields $\mu / \mathrm{Er}^{3+}=5.64 \mu_{\mathrm{B}}$, in good agreement with the magnitudes measured by neutron diffraction studies.

In antiferromagnets, the position of $T_{N}$ is accurately determined not by the peak in the $\chi$ vs. $T$ data but by the maximum in $d(\chi \mathrm{T}) / d \mathrm{~T}$. The physical basis comes for this from the fact that $\chi \mathrm{T}$ represents magnetic energy [48, 49]. The variation of $d(\chi \mathrm{T}) / d \mathrm{~T}$ vs. T for the ZFC data in Fig. 5.9 yields $\mathrm{T}_{\mathrm{N}}=3.3 \mathrm{~K}$ for both the $b-\mathrm{Er}_{2} \mathrm{O}_{3}$ and the $\mathrm{n}-\mathrm{Er}_{2} \mathrm{O}_{3}$ samples. From the peak in the temperature dependence of the heat capacity, Tang et al. [62] also reported $\mathrm{T}_{\mathrm{N}}=3.3 \mathrm{~K}$. The data of Tang et al. [62] on the temperature dependence of heat capacity in $\mathrm{Er}_{2} \mathrm{O}_{3}$ is also displayed in Fig. 5.9 for comparison purposes. To determine the $\mathrm{H}$-dependence of $\mathrm{T}_{\mathrm{N}}$ from the data of Fig. 5.7, the plots of $d(\chi \mathrm{T}) / d \mathrm{~T}$ vs. $\mathrm{T}$ for different $\mathrm{H}$ are shown in Fig. 5.10. In the inset of Fig. 5.11, we show the fit of the $\mathrm{T}_{\mathrm{N}} \mathrm{vs}$. $\mathrm{H}$ variation to the Eq.: $\mathrm{T}_{\mathrm{N}}(\mathrm{H})=\mathrm{T}_{\mathrm{N}}(0)-\mathrm{D}_{1} \mathrm{H}^{2}$, , with $\mathrm{D}_{1}=7.3 \times 10^{-9} \mathrm{~K} / \mathrm{Oe}^{2}$. A similar $\mathrm{T}_{\mathrm{N}} \mathrm{vs}$. $\mathrm{H}$ variation has been reported in the uniaxial antiferromagnet $\mathrm{MnF}_{2}$ but with $\mathrm{D}_{1}=1.59 \times 10^{-10} \mathrm{~K} / \mathrm{Oe}^{2}$ [69]. For $\mathrm{H}$ parallel to the easy axis, molecular field theory (MFT) yields the following Eq. for $\mathrm{D}_{1}$ : 


$$
D_{1}=\frac{g^{2} \mu_{B}^{2}\left(2 S^{2}+2 S+1\right)}{\left(40 k_{B}^{2} T_{N}\right)}
$$

For $\mathrm{Mn}^{2+}$ ions in $\mathrm{MnF}_{2}$ with $\mathrm{T}_{\mathrm{N}}=67.3 \mathrm{~K}, \mathrm{~g}=2$ and $\mathrm{S}=5 / 2$. Using these magnitudes in Eq. (5.1) yields $\mathrm{D}_{1}=1.24 \times 10^{-10} \mathrm{~K} / \mathrm{Oe}^{2}$, a value about $28 \%$ smaller the experimental $\mathrm{D}_{1}$ quoted above. For $\mathrm{Er}^{3+}$ ions in $\mathrm{Er}_{2} \mathrm{O}_{3}$ with $\mathrm{T}_{\mathrm{N}}=3.3 \mathrm{~K}, \mathrm{~g}=6.627$ and $\mathrm{S}=1 / 2$ as discussed later. Substituting these magnitudes in Eq. (5.1) yields $\mathrm{D}_{1}=3.36 \times 10^{-9} \mathrm{~K} / \mathrm{Oe}^{2}$. As in $\mathrm{MnF}_{2}$, this calculated magnitude of $\mathrm{D}_{1}$ in $\mathrm{Er}_{2} \mathrm{O}_{3}$ is somewhat smaller than the experimental $\mathrm{D}_{1}=7.3 \times 10^{-9} \mathrm{~K} / \mathrm{Oe}^{2}$, perhaps due to the approximations of the MFT. However, it is satisfying to note that with the MFT, it is possible to explain a factor of about 45 difference in the measured values of $\mathrm{D}_{1}$ in $\mathrm{Er}_{2} \mathrm{O}_{3}$ and $\mathrm{MnF}_{2}$.

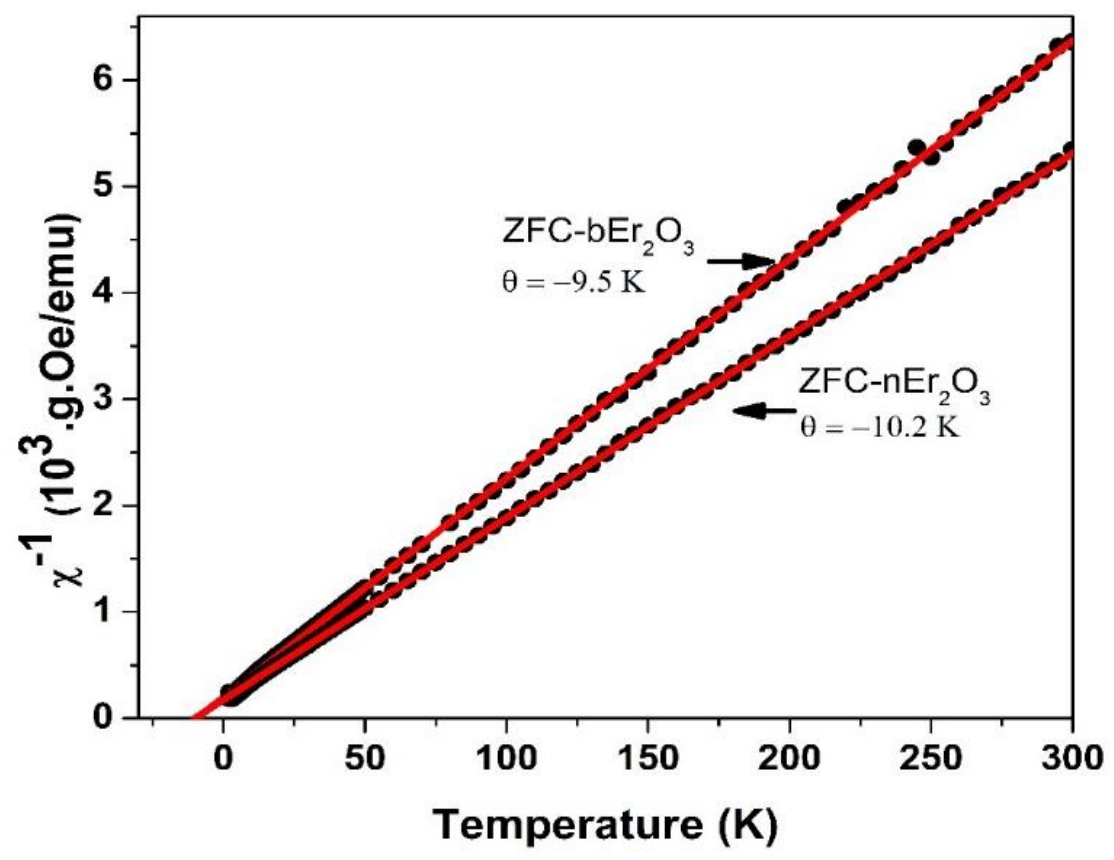

Figure 5.8: Plots of inverse susceptibility vs. temperature; solid lines fits to the Curie-Weiss law. 


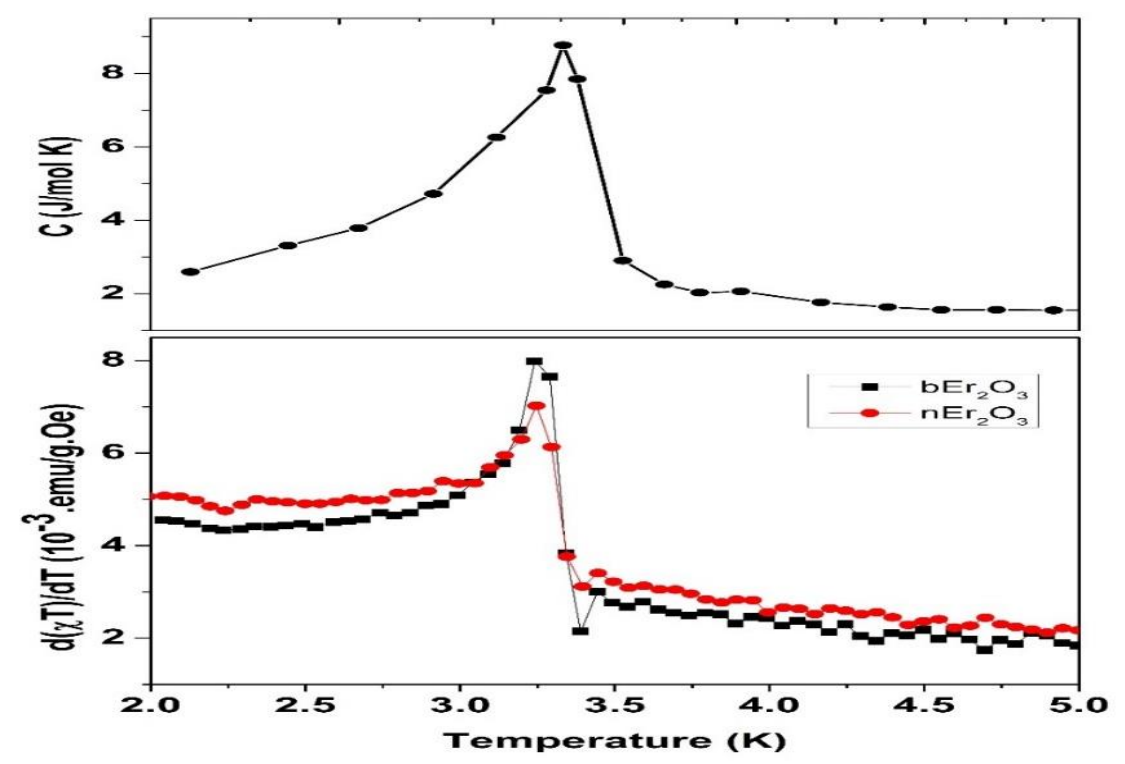

Figure 5.9: Plots of the computed $d(\chi \mathrm{T}) / d \mathrm{~T}$ vs. $\mathrm{T}$ obtained from the ZFC data near $\mathrm{T}_{\mathrm{N}}$ (Fig. 5.3) for the two samples of $\mathrm{Er}_{2} \mathrm{O}_{3}$. Top panel shows the specific heat vs. temperature data of Tang et al. [62].

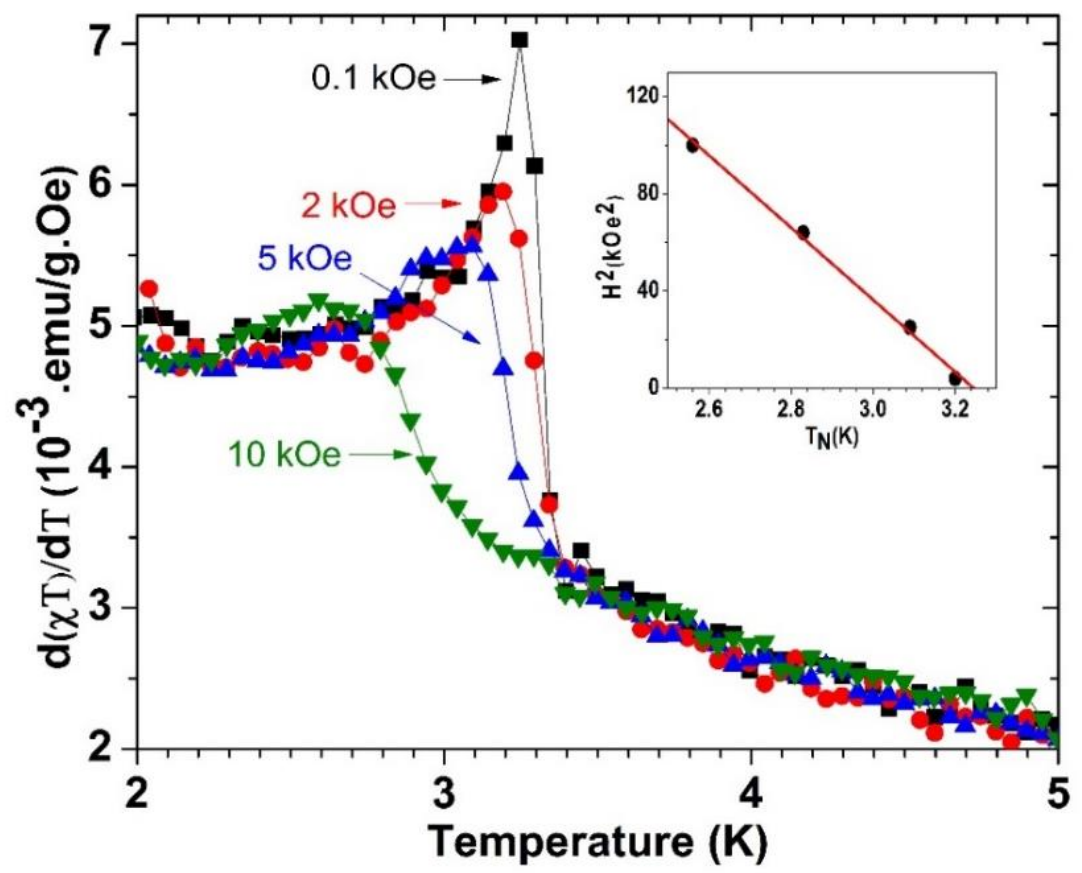

Figure 5.10: Data of Fig.5.6 are used to compute and plot $d(\chi \mathrm{T}) / d \mathrm{~T}$ vs. T curves for determining $\mathrm{T}_{\mathrm{N}}$ for different $H$. The inset shows the variation of $\mathrm{T}_{\mathrm{N}}$ vs. $\mathrm{H}^{2}$ to determine $\mathrm{D}_{1}$ of Eq. (5.1). 


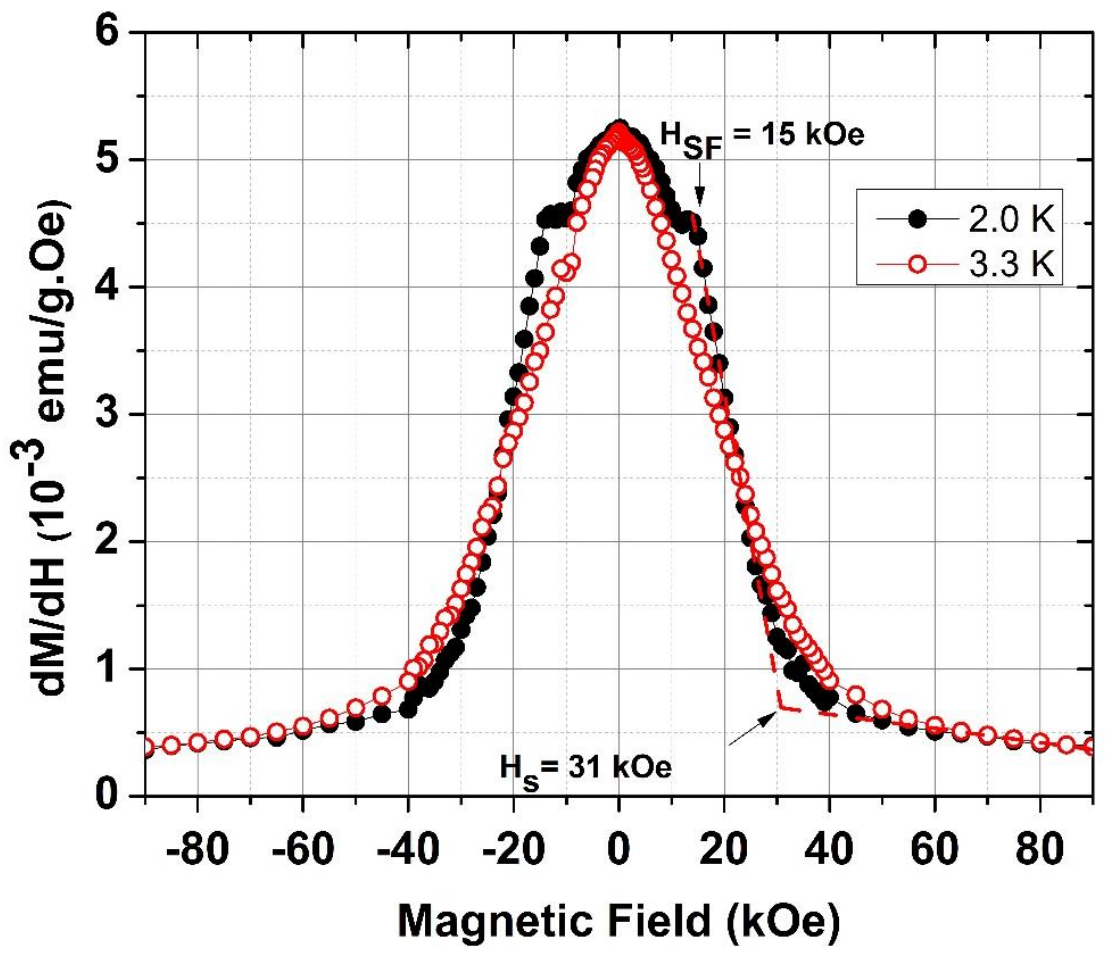

Figure 5.11: Computed $d \mathrm{M} / d \mathrm{H}$ vs. $\mathrm{H}$ for the data of Fig. 5 at $\mathrm{T}=2.0 \mathrm{~K}$ and $3.3 \mathrm{~K}$. Dashed lines are linear fits yielding $\mathrm{H}_{\mathrm{S}}=31 \mathrm{kOe}$ for the data at $\mathrm{T}=2.0 \mathrm{~K}$.

Next we consider the analysis of the data of M vs. H for different temperatures. Since the analysis of the plots of Fig. 5.7 was not conclusive, the plots of the computed $d \mathrm{M} / d \mathrm{H}$ vs. $\mathrm{H}$ for two temperatures of $2.0 \mathrm{~K}$ and $3.3 \mathrm{~K}$ are shown in Fig. 5.11. A peak in $d \mathrm{M} / d \mathrm{H}$ at $\mathrm{H}_{\mathrm{C}} \simeq 15 \mathrm{kOe}$ is clearly observed for the data at $2.0 \mathrm{~K}$ only whereas such a peak is absent at $3.3 \mathrm{~K}$. At $2 \mathrm{~K}$, the saturation in $d \mathrm{M}$ $/ d \mathrm{H}$ occurs at $\mathrm{H}_{\mathrm{S}} \simeq 31 \mathrm{kOe}$ as indicated by the dotted lines in Fig. 5.11. Similar analysis of $d \mathrm{M} / d \mathrm{H}$ vs. $\mathrm{H}$ variations at different $\mathrm{T}$ is shown in the plots of Fig. 5.12 where it is evident that the position of the critical field $\mathrm{H}_{\mathrm{C}}$ marked by the dotted line in Fig. 5.12 decreases with increase in $\mathrm{T}$, approaching zero at $\mathrm{T}_{\mathrm{N}}$. Thus $\mathrm{H}_{\mathrm{C}}$ so determined can be unambiguously associated with the antiferromagnetic ordering of the system. In the following it is shown that $\mathrm{H}_{\mathrm{C}}=\mathrm{H}_{\mathrm{SF}}$ represents the spin-flop transition in $\mathrm{Er}_{2} \mathrm{O}_{3}$.

If all the $32 \mathrm{Er}^{3+}$ in the cubic unit with side $\mathrm{a}=10.55 \AA$ were aligned parallel to each other with magnetic moment $\mu=5.64 \mu_{\mathrm{B}}$, with zero-point spin-deviations taken into account, the expected value of the saturation magnetization $\mathrm{M}_{\mathrm{S}}$ of $\mathrm{Er}_{2} \mathrm{O}_{3}$ can be calculated. This calculation yields $\mathrm{M}_{\mathrm{S}}=165 \mathrm{emu} / \mathrm{g}$. The data in Fig. 5.4 shows that at $2 \mathrm{~K}$ and $90 \mathrm{kOe}, \mathrm{M}=150 \mathrm{emu} / \mathrm{g}$ but $\mathrm{M}$ is not saturated even at 90 
kOe. By plotting $M$ vs. 1/H yields $M_{S} \sim 173 \mathrm{emu} / \mathrm{g}$ is estimated in the limit of $\mathrm{H} \rightarrow \infty$ (see Fig. 5.5). This magnitude is in good agreement with the above calculated value of $\mathrm{M}_{\mathrm{S}}$ for ferromagnetic ordering.

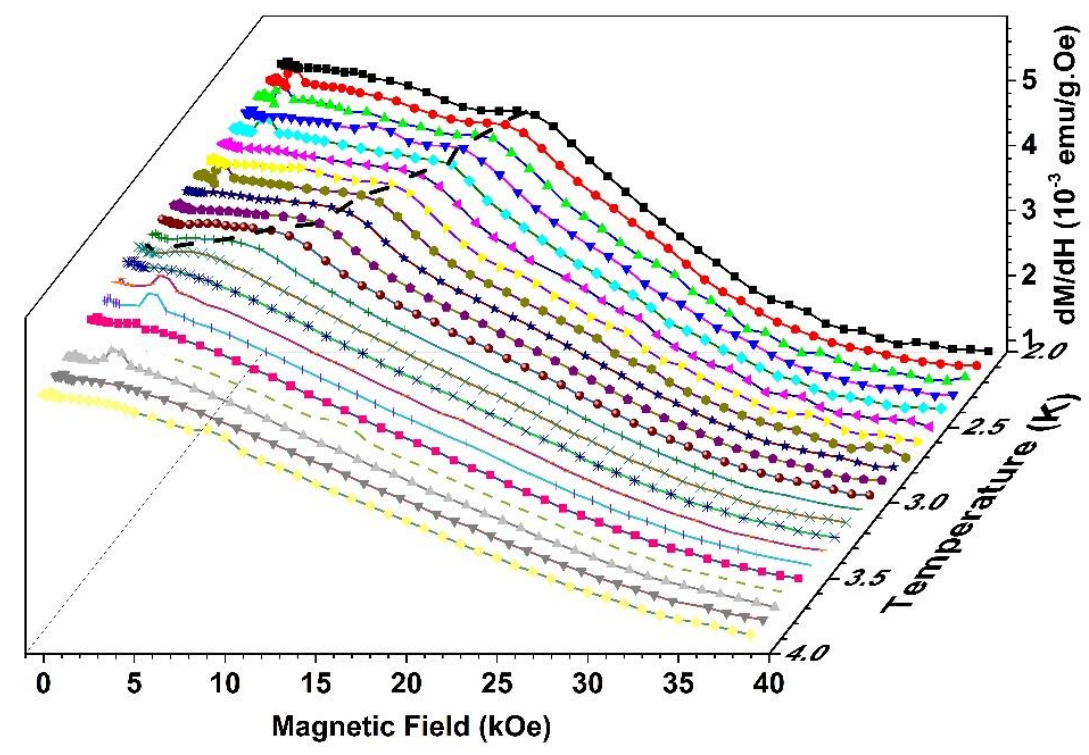

Figure 5.12: Plots of $\mathrm{dM} / \mathrm{dH}$ vs. $\mathrm{H}$ computed from the data of $\mathrm{M}$ vs. $\mathrm{H}$ of Fig. 5.6 in the range of 2.0$4.0 \mathrm{~K}$ taken at $0.1 \mathrm{~K}$ steps. The dashed lines connect the crests depicting the spin-flop field $\mathrm{H}_{\mathrm{SF}}$ and its temperature dependence.

Next, the nature of the field-induced transitions at $\mathrm{H}_{\mathrm{C}} \simeq 15 \mathrm{kOe}$ and $\mathrm{H}_{\mathrm{S}} \simeq 31 \mathrm{kOe}$ in $\mathrm{Er}_{2} \mathrm{O}_{3}$ is explored in the following analysis. Two kinds of magnetic-field-induced transitions are known in antiferromagnets below $\mathrm{T}_{\mathrm{N}}$. The first kind of transition involves spin-flip observed in layered systems with ferromagnetically aligned layers but neighboring layers aligned antiferromagnetically resulting in overall AFM order. However, the in-plane FM exchange coupling between the magnetic ions in such systems is much stronger than the interlayer AFM coupling so that for $\mathrm{H}>\mathrm{H}_{\mathrm{C}}$, spins in the neighboring layers flip to align along the direction of the applied $\mathrm{H}$ yielding a ferromagnet but without change in the easy direction. Such an AFM to FM magnetic-field-induced transition is often termed a metamagnetic transition, and it has been reported in the layered systems $\mathrm{FeCl}_{2}$ and $\mathrm{FeBr}_{2}$ [70, 71] and more recently in the layered $\beta-\mathrm{Ni}(\mathrm{OH})_{2}$ with $\mathrm{H}_{\mathrm{C}} \simeq 55 \mathrm{kOe}$ [72]. The spin-flip transitions usually have hysteresis and a first order character. Since $\mathrm{Er}_{2} \mathrm{O}_{3}$ is not a layered system and it has instead a complex magnetic ordering shown in Fig.5.2, it is inferred that the H-induced transition in $\operatorname{Er}_{2} \mathrm{O}_{3}$ cannot be spinflip type. 
Spin-flop is the second kind of $\mathrm{H}$-induced transition in antiferromagnets. This transition occurs for $\mathrm{H}$ applied along the easy direction in systems in which $\mathrm{H}_{e x}>\mathrm{H}_{\mathrm{A}}$ where $\mathrm{H}_{e x}\left(\mathrm{H}_{\mathrm{A}}\right)$ is the equivalent exchange (anisotropy) magnetic field. This transition occurs at the magnetic field $\mathrm{H}_{\mathrm{SF}}=\left(2 \mathrm{H}_{e x} \mathrm{H}_{\mathrm{A}}\right)^{1 / 2}$ $[53,69,73]$ so that for $\mathrm{H}>\mathrm{H}_{\mathrm{SF}}$ the spins flop by $90^{\circ}$ to the hard direction. In the flopped state just above $\mathrm{H}>\mathrm{H}_{\mathrm{SF}}$, the spins are still aligned antiferromagnetically but now along the hard direction with $\chi$ for $\mathrm{H}>\mathrm{H}_{\mathrm{SF}}$ greater than $\chi$ for $\mathrm{H}<\mathrm{H}_{\mathrm{SF}}$. The observations in the antiferromagnet $\mathrm{MnF}_{2}$ with $\mathrm{T}_{\mathrm{N}}=67.3$ $\mathrm{K}$ are typical of the spin-flop transition [53, 69]. In antiferromagnetic super-lattices, two spin-flop transitions have been reported; spin-flop of the surface layer spins occurring at a lower field followed by the spin-flop of the bulk system at a higher field $[72,73]$. Further increases in $\mathrm{H}$ above $\mathrm{H}_{\mathrm{SF}}$ continually rotate the spins to bring them parallel to $\mathrm{H}$ so that at a specific field $\mathrm{H}_{\mathrm{S}}=\mathrm{M}_{\mathrm{S}} / \chi_{\perp}$, the system is affectively a forced ferromagnet [72]. Here $\chi_{\perp}$ is the magnetic susceptibility along the hard direction. Usually the transition at $\mathrm{H}_{\mathrm{SF}}$ is a first order transition accompanied by hysteresis whereas the transition at $\mathrm{H}_{\mathrm{S}}$ is more diffused. Using $\mathrm{M}_{\mathrm{S}}=165 \mathrm{emu} / \mathrm{g}$ expected for ferromagnetic ordering and $\chi_{\perp}=\chi\left(\mathrm{T}_{\mathrm{N}}\right)=$ $5.4 \times 10^{-3} \mathrm{emu} / \mathrm{g} \cdot$ Oe in in $\mathrm{Er}_{2} \mathrm{O}_{3}$ yields $\mathrm{H}_{\mathrm{S}}=30.5 \mathrm{kOe}$ in close agreement with $\mathrm{H}_{\mathrm{S}} \sim 31 \mathrm{kOe}$ observed experimentally in $\mathrm{Er}_{2} \mathrm{O}_{3}$. The relation $\chi_{\perp}=\chi\left(\mathrm{T}_{\mathrm{N}}\right)$ used in the above calculations is found to be a good approximation in antiferromagnets like $\mathrm{MnF}_{2}$ [69] and $\mathrm{RbMnF}_{2}$ [74]. These results lead to conclude that in $\mathrm{Er}_{2} \mathrm{O}_{3} \mathrm{H}_{\mathrm{S}} \sim 31 \mathrm{kOe}$ is sufficient to align the spins along the applied magnetic field by overcoming exchange coupling and so $\mathrm{H}_{e x}=\mathrm{H}_{\mathrm{S}}$ can be inferred. For $\mathrm{H}>\mathrm{H}_{e x}$ the spins will tend to align along the $\mathrm{H}$-direction even for $\mathrm{T}>\mathrm{T}_{\mathrm{N}}$ except for some disorder due to thermal energy. However, for $\mathrm{T}>\mathrm{T}_{\mathrm{N}}$, the magnitude of $\mathrm{H}_{\mathrm{S}}$ above which $\mathrm{M}$ is nearly saturated are higher than $30 \mathrm{kOe}$ but difficult to determine accurately.

Based on the above analysis and discussion, it is proposed that in $\operatorname{Er}_{2} \mathrm{O}_{3}$, the transition at $\mathrm{H}_{\mathrm{C}} \simeq$ $15 \mathrm{kOe}$ at $2 \mathrm{~K}$ and decreasing to $\mathrm{H}_{\mathrm{C}}=0$ at $\mathrm{T}_{\mathrm{N}}$ (Fig. 5.12) is the spin-flop transition. In $\mathrm{Er}_{2} \mathrm{O}_{3}$, the signatures of this transition are somewhat diffused first because the sample was in the powdered form making it impossible to align the sample along the easy direction and second because in this system, there are multiple spin easy axes as stated earlier and evident in Fig. 5.2. This situation is analogous to the case of cubic antiferromagnet $\mathrm{RbMnF}_{3}$ with four equivalent $\langle 111>$ easy axis. In this case also the spin-flop transition was also observed to be very diffused and weak [74-76]. Using $\mathrm{H}_{e x}=\mathrm{H}_{\mathrm{S}}=31 \mathrm{kOe}$ in $\mathrm{H}_{\mathrm{SF}}=\left(2 \mathrm{H}_{e x} \mathrm{H}_{\mathrm{A}}\right)^{1 / 2}=15 \mathrm{kOe}$ yields $\mathrm{H}_{\mathrm{A}}=3.63 \mathrm{kOe}$ as the effective anisotropy field in $\mathrm{Er}_{2} \mathrm{O}_{3}$. $\mathrm{This}$ magnitude of $\mathrm{H}_{\mathrm{A}}$ is in line with the dipole energy calculated for the $\mathrm{C}_{2}$ site by Moon et al. [55].

Both $\mathrm{H}_{\mathrm{ex}}$ and $\mathrm{H}_{\mathrm{A}}$ are proportional to $\langle\mathrm{S}\rangle$. Therefore the temperature dependence of $\mathrm{H}_{\mathrm{SF}}=$ $\left(2 \mathrm{H}_{e x} \mathrm{H}_{\mathrm{A}}\right)^{1 / 2}$ is expected to vary as $\langle\mathrm{S}\rangle$, the order parameter $[69,72,77]$. For this reason, $\mathrm{H}_{\mathrm{SF}}$ approaching zero at $\mathrm{T}_{\mathrm{N}}$ in Fig. 5.13 is quite understandable. Based on the molecular field theory, the quantitative 
temperature dependence of $\mathrm{H}_{\mathrm{SF}}$ is compared in Fig. 5.13 with that of the Brillouin function for $\mathrm{S}=1 / 2$ valid for $\mathrm{Er}^{3+}$ ions in $\mathrm{Er}_{2} \mathrm{O}_{3}$. Specifically, for $\mathrm{S}=1 / 2$, the Brillouin function variation reduces to the following equation for the variation of the reduced spin- flop field $h=\mathrm{H}_{\mathrm{SF}}(\mathrm{T}) / \mathrm{H}_{\mathrm{SF}}(0)$ in terms of the reduced Nèel temperature $t=\left(T / T_{N}\right)[46]$ :

$$
h=\tanh (h / t)
$$

The solid line in Fig. 5.13 is based on the solution of Eq. (5.2) for h vs. t. Such a variation has been reported in other $\mathrm{S}=1 / 2$ systems [46].

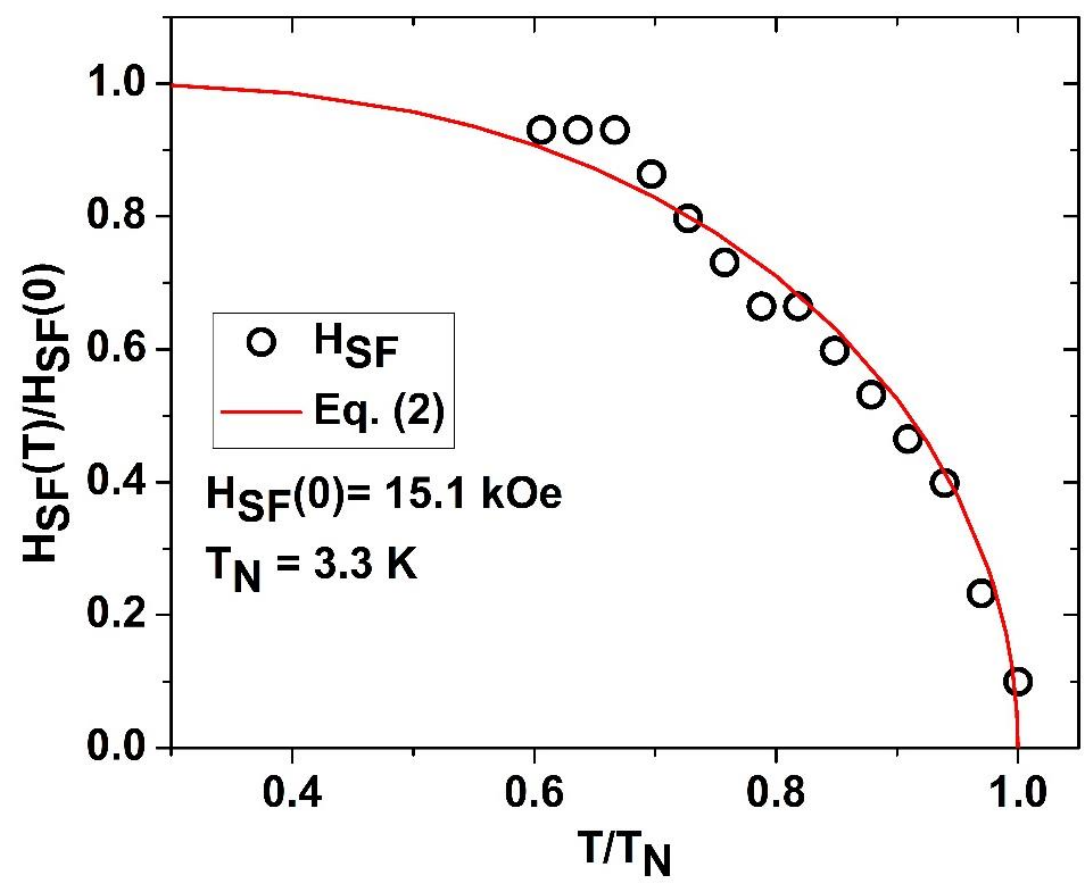

Figure 5.13: Variation of reduced spin-flop field $h=\mathrm{H}_{\mathrm{SF}}(\mathrm{T}) / \mathrm{H}_{\mathrm{SF}}(0)$ vs. the reduced Nèel temperature $\mathrm{t}$ $=\mathrm{T} / \mathrm{T}_{\mathrm{N}}$. The solid line is based on the solution to Eq. (5.2).

\subsection{Calculations of the Exchange Constants:}

In this section, exchange constants responsible for antiferromagnetic ordering in $\operatorname{Er}_{2} \mathrm{O}_{3}$ are estimated using molecular field theory even though the AFM ordering in $\mathrm{Er}_{2} \mathrm{O}_{3}$ as revealed by neutron diffraction studies of Moon et al. [55] and shown in Fig. 2 is quite complicated in that there is no unique direction of magnetic ordering. Instead, the moments on the $C_{2}$ sites are aligned along the three <100> axes depending upon the local symmetry axis and the moments on the $\mathrm{C}_{3 \mathrm{i}}$ sites are along the <111> axis. Ignoring the slight displacement (1/30 of lattice constant) of the $\mathrm{Er}^{3+}$ ions on the $\mathrm{C}_{2}$ sites from the positions shown in Fig. 5.1, there are $12 \mathrm{nn}$ (nearest-neighbors) of each $\mathrm{Er}^{3+}$ ion from the $\mathrm{C}_{2}$ sites $\left(\mathrm{Z}_{1}\right.$ 
$=12$ ) and 4 nnn (next-nearest- neighbors) neighbors on the $C_{3 i}$ sites $\left(Z_{2}=4\right)$. We label the exchange interactions with the $n n$ and nnn as $\mathbf{J}_{1}$ and $\mathbf{J}_{2}$ respectively and assume Heisenberg exchange interaction with the Hamiltonian:

$$
\mathcal{H}=-2 \sum_{i j} J_{i j} \overrightarrow{S_{l}} \cdot \overrightarrow{S_{J}}-g \mu_{B} \vec{H} \cdot \sum_{j} \overrightarrow{S_{J}}
$$

Using the two sub-lattice model with the $\mathrm{Er}^{3+}$ ions on the $\mathrm{C}_{2}$ sites $\left(\mathrm{C}_{3 \mathrm{i}}\right.$ sites $)$ making up sublattice 1 (sublattice 2) and the molecular-field approximation, the following expressions for $\theta$ and $\mathrm{T}_{\mathrm{N}}$ then follow $[46,72]$ :

$$
\begin{aligned}
& 3 k_{B} \theta=2 S(S+1)\left[J_{1} Z_{1}+J_{2} Z_{2}\right] \\
& 3 k_{B} T_{N}=2 S(S+1)\left[J_{1} Z_{1}-J_{2} Z_{2}\right]
\end{aligned}
$$

Here $S=1 / 2$ is the effective spin for $\mathrm{Er}^{3+}$. Using $\theta=-10.2 \mathrm{~K}$ and $\mathrm{T}_{\mathrm{N}}=3.3 \mathrm{~K}$ determined earlier in Eqs. (5.4) and (5.5) yields $\mathrm{J}_{1} / \mathrm{k}_{\mathrm{B}}=-0.57 \mathrm{~K}$ and $\mathrm{J}_{2} / \mathrm{k}_{\mathrm{B}}=-3.38 \mathrm{~K}$. The sign convention used here implies that both $J_{1}$ and $J_{2}$ are antiferromagnetic. The magnitude of the ratio $\theta / T_{N}=3.1$ different from unity observed in $\mathrm{Er}_{2} \mathrm{O}_{3}$ implies the presence of more than one exchange constants. This is quite evident by examining Eqs. (5.4) and (5.5).

We use the same procedure as used in the successful calculation of magnetic field needed for the spin-flip transition in $\beta-\mathrm{Ni}(\mathrm{OH})_{2}$ [72] to derive an expression for $\mathrm{H}_{\mathrm{S}}$ in $\mathrm{Er}_{2} \mathrm{O}_{3}$ using Hamiltonian in Eq.(3) and the magnetic ordering observed by neutron diffraction shown in Fig.5.2. For a given $\mathrm{Er}^{3+}$ on the $\mathrm{C}_{2}$ site, there are $4 \mathrm{nn}$ with opposite spin and another $8 \mathrm{nn}$ with spin aligned perpendicular to it. The four nnn spin on the $\mathrm{C}_{3 \mathrm{i}}$ sites are aligned at an angle of $54.7^{\circ}$. Using these facts, the AFM state in applied $\mathrm{H}=0$ has the energy

$$
E_{\uparrow \downarrow}=-2 N S^{2}\left[-4 J_{1}+4 J_{2} \cos 54.7^{\circ}\right]
$$

For $\mathrm{H}=\mathrm{H}_{\mathrm{s}}$, the system is in the FM state with the energy

$$
E_{\uparrow \downarrow}=-2 N S^{2}\left[12 J_{1}+4 J_{2}\right]-N S g \mu_{B} H_{S}
$$

Equating equations (5.6) to (5.7) yields the critical field:

$$
H_{S}=-2\langle S\rangle\left(16 J_{1}+1.69 J_{2}\right) / g \mu_{B}
$$

Here $g$ is the effective $g$-value of $\mathrm{Er}^{3+}$ in $\mathrm{Er}_{2} \mathrm{O}_{3}$. The magnitudes of $\mathrm{g}$ values determined by electron paramagnetic resonance (EPR) of $\mathrm{Er}^{3+}$ ions doped in iso-structural diamagnetic $\mathrm{Y}_{2} \mathrm{O}_{3}$, are: $\mathrm{g}_{\mathrm{x}}=12.3, \mathrm{~g}_{\mathrm{y}}$ $=1.6, \mathrm{~g}_{\mathrm{z}}=4.9$ for the $\mathrm{C}_{2}$ sites and $\mathrm{g}_{\|}=12.2$ and $\mathrm{g}_{\perp}=3.3$ for the $\mathrm{C}_{3 \mathrm{i}}$ sites [78]. Averaging over both the 
$\mathrm{C}_{2}$ and $\mathrm{C}_{3 \mathrm{i}}$ sites, these magnitudes yield algebraic average $\mathrm{g}=6.267$. In Eq. (5.8) using $\mathrm{J}_{1} / \mathrm{k}_{\mathrm{B}}=-0.57 \mathrm{~K}$ and $\mathrm{J}_{2} / \mathrm{k}_{\mathrm{B}}=-3.38 \mathrm{~K}$ determined earlier and $<\mathrm{S}>=0.422$ with zero-point spin deviations taken into account yields $\mathrm{H}_{\mathrm{S}}=29.8 \mathrm{kOe}$ for $\mathrm{g}=6.267$. This calculated magnitude of $\mathrm{H}_{\mathrm{S}}$ is in excellent agreement with the measured value of $\mathrm{H}_{\mathrm{S}} \simeq 31 \mathrm{kOe}$. This good agreement provides considerable confidence in the interpretation of $\mathrm{H}_{\mathrm{S}}$ and the estimated values of exchange constants $\mathrm{J}_{1}$ and $\mathbf{J}_{2}$.

\subsection{Concluding Remarks:}

In this Chapter, analysis, discussion and interpretation of the data on the temperature and magnetic field dependence of the magnetization in powder samples of $\mathrm{Er}_{2} \mathrm{O}_{3}$ have been presented. Based on this analysis, new results on the magnetic parameters associated with the complex antiferromagnetic ordering in this system below its $\mathrm{T}_{\mathrm{N}}=3.3 \mathrm{~K}$ have been presented. New results presented here include evaluations of the $n n$ and nnn exchange constants $J_{1}$ and $J_{2}$ from the fit of the experimental data for $T$

$>\mathrm{T}_{\mathrm{N}}$ to the Curie-Weiss law using the molecular-field model and the observation and satisfactory interpretations of the two magnetic field induced transitions, viz. the spin-flop transition at $\mathrm{H}_{\mathrm{SF}}=15$ kOe and the transition to ferromagnetism above $\mathrm{H}_{\mathrm{S}} \simeq 31 \mathrm{kOe}$. An additional new contribution is the derivation of an expression for $\mathrm{H}_{\mathrm{S}}$ in terms of $\mathrm{J}_{1}$ and $\mathrm{J}_{2}$ which yields the magnitude of $\mathrm{H}_{\mathrm{S}}$ in excellent agreement with the measured value. Another important result is the magnetic field dependence of $T_{N}$ which is found to fit the Eq.: $\mathrm{T}_{\mathrm{N}}(\mathrm{H})=\mathrm{T}_{\mathrm{N}}(0)-\mathrm{D}_{1} \mathrm{H}^{2}$ expected for antiferromagnets; The large difference in the magnitudes of $\mathrm{D}_{1}$ for $\mathrm{Er}_{2} \mathrm{O}_{3}$ and the uniaxial antiferromagnet $\mathrm{MnF}_{2}$ has also been explained. Finally, the temperature dependence of $\mathrm{H}_{\mathrm{SF}}$ is shown to coincide with the temperature dependence of the normalized Brillouin function for spin $\mathrm{S}=1 / 2$ valid for $\mathrm{Er}_{2} \mathrm{O}_{3}$. Although the presence of multiple spin easy axes in $\mathrm{Er}_{2} \mathrm{O}_{3}$ makes the spin-flop transition quite diffused and so harder to observe, clear observations of this transition and its temperature dependence (Figs.5.11 to 5.13) are reported and interpreted in this chapter. 


\section{CHAPTER 6 - Conclusions and Publications}

\subsection{Conclusions:}

The objectives of this research were to determine the effects of Erbium in Erbium-alloyed AlN thin films on their structural, piezoelectric and magnetic properties. For this purpose, Erbium-alloyed AlN thin films using Aluminum-Er alloyed sputtering targets with Erbium concentration of 0, 1, 3 and 4 atomic percent were deposited on (001) p-type Si substrates by reactive magnetron sputtering. In this work, the piezoelectric measurements carried out on AlN and AlN:Er thin films showed that for the thin films deposited using Erbium concentration of $3 \%, \mathrm{~d}_{33}$ coefficient increased by more than two fold. Based on XRD measurements, the increased magnitude of $d_{33}$ is attributed to the absence of trace peaks

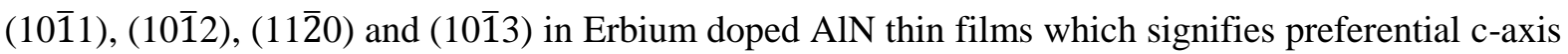
deposition of AlN:Er. The Variable Angle Spectroscopic Ellipsometer Measurements (VASE) on AlN:Er thin films show refractive index to be in the range of 2.0-2.1 for films used for piezoelectric studies. This magnitude of refractive index agrees with the refractive index measured in epitaxial grown thin films showing that AlN:Er films investigated here have high structural order. The preferential caxis orientation and high structure order are likely responsible for improved piezoelectric properties of AlN on alloying with Erbium.

Elemental compositions of the thin films at the surface and in the bulk were determined using XPS measurements. XPS measurements confirmed the presence of Erbium in the thin films. The results obtained from these measurements also showed the presence of significant amount of oxygen with higher values at the surface of the thin films than in the bulk.

The next important result is on the determination of the electronic state of Erbium in the AlN:Er films. For this purpose, detailed investigations of the temperature and magnetic field variations of the magnetic properties were carried. The analysis of the results on the temperature and magnetic field variations of the magnetization in a sputtered film of $\mathrm{AlN}$ :Er presented here has established $\mathrm{Er}^{3+}$ as the electronic state of Er. Even though some oxygen is present in the thin film, it is present as Al-O in a thin layer on the surface of the film and not as $\mathrm{Er}_{2} \mathrm{O}_{3}$. These results have established that $\mathrm{Er}^{3+}$ substitutes for $\mathrm{Al}^{3+}$ during sputtering and preferentially aligns the film with c-axis normal to the film. The results presented here have also confirmed the usefulness of magnetic measurements for determining the electronic states of magnetic additives in non-magnetic hosts. The concentration $\mathrm{x}=1.08 \% \mathrm{of} \mathrm{Er}^{3+}$ determined from analysis of the magnetic data is in good agreement with $\mathrm{x}=0.94 \pm 0.20 \%$ determined using XPS.

Analysis of the data on the temperature and magnetic field dependence of the magnetization in powder samples of $\mathrm{Er}_{2} \mathrm{O}_{3}$ have been used here to determine the magnetic parameters associated with 
the complex antiferromagnetic ordering in this system below its $T_{N}=3.3 \mathrm{~K}$. New results presented in this work include evaluations of the $n n$ and nnn exchange constants $J_{1}$ and $J_{2}$ from the fit of the experimental data for $\mathrm{T}>\mathrm{T}_{\mathrm{N}}$ to the Curie-Weiss law using the molecular-field model and the observation and satisfactory interpretations of the two magnetic field induced transitions. These transitions are the spin-flop transition at $\mathrm{H}_{\mathrm{SF}}=15 \mathrm{kOe}$ and the transition to ferromagnetism above $\mathrm{H}_{\mathrm{S}}$ $\simeq 31 \mathrm{kOe}$. An expression for $\mathrm{H}_{\mathrm{S}}$ is derived in terms of $\mathrm{J}_{1}$ and $\mathrm{J}_{2}$ which yields the magnitude of $\mathrm{H}_{\mathrm{S}}$ in excellent agreement with the measured value. The magnetic field dependence of $T_{N}$ is found to fit the Eq.: $\mathrm{T}_{\mathrm{N}}(\mathrm{H})=\mathrm{T}_{\mathrm{N}}(0)-\mathrm{D}_{1} \mathrm{H}^{2}$ expected for antiferromagnets and the large difference in the magnitudes of $\mathrm{D}_{1}$ for $\mathrm{Er}_{2} \mathrm{O}_{3}$ and the uniaxial antiferromagnet $\mathrm{MnF}_{2}$ has been explained. Finally, the temperature dependence of $\mathrm{H}_{\mathrm{SF}}$ is shown to coincide with the temperature dependence of the normalized Brillouin function for spin $\mathrm{S}=1 / 2$ valid for $\mathrm{Er}_{2} \mathrm{O}_{3}$. Although the presence of multiple spin easy axes in $\mathrm{Er}_{2} \mathrm{O}_{3}$ makes the spin-flop transition quite diffused and so harder to observe, clear observations of this transition and its temperature dependence (Figs. 5.11 to 5.13) are reported using the computed dM/dH vs. H plots. Satisfactory interpretation of the transitions has also been presented. It can be stated with confidence that the magnetic studies presented here on $\mathrm{Er}_{2} \mathrm{O}_{3}$ has provided near complete understanding of the nature of magnetic ordering in this complex system.

\subsection{Publications of the Author Related to this Dissertation:}

Descriptions and discussion of the results presented in this dissertation have appeared in literature with details given below.

- V. Narang, D. Korakakis, M.S. Seehra; Electronic state of Er in sputtered AlN:Er films determined by magnetic measurements. Journal of Applied Physics, 116, 213911 (2014).

- V. Narang, D. Korakakis, M.S. Seehra; Nature of magnetism and magnetic field induced transitions in non-collinear antiferromagnet $\mathrm{Er}_{2} \mathrm{O}_{3}$; Journal of Magnetism and Magnetic Materials, 368, 353 (2014).

- V. Narang, D. Korakakis; Effects of Erbium alloying on the structural and piezoelectric properties of Aluminum Nitride thin films annealed under extreme thermal conditions; Mater. Res. Soc. Symp. Proc. Vol. 1519 (2013). 


\subsection{Other Publications of the Author:}

- S. Thota, V. Narang, S. Nayak, S. Sambasivam, B. C. Choi, T. Sarkar, M. S. Anderson, R Mathieu and M. S. Seehra; On the nature of magnetic state in the spinel $\mathrm{Co}_{2} \mathrm{SnO}_{4}$; Journal of Physics: Condensed Matter, 27, 166001 (2015).

- R. Rahimi, V.Narang, D. Korakakis; Optical and Morphological Studies of Thermally Evaporated PTCDI-C8 Thin Films for Organic Solar Cell Applications; International Journal of Photoenergy, Article ID 205105 (2013).

- R. Rahimi, A. Roberts, V.Narang, V. Kumbham, D. Korakakis; Study of the effect of the charge transport layer in the electrical characteristics of the organic photovoltaics; Optical Materials, 35, 1077 (2013).

- R. Rahimi, A. Roberts, V.Narang, D. Korakakis; Investigate the role of the active layers' structures and morphology in the performance of the organic solar cell devices; Applied Physics Letters, 102, 073105 (2013).

- J. Justice, L.E. Rodak, V. Narang, K. Lee, L.A. Hornak, D. Korakakis; Characterization of Group III-Nitride Based Surface Acoustic Wave Devices for High Temperature Applications; Mater. Res. Soc. Symp. Proc. Vol. 1299 (2011).

- V. Kumbham, S. Kuchibhatla, K. Lee, L.E. Rodak, V. Narang, D. Korakakis, L.A. Hornak; Ohmic and Highly Reflective Ag based contacts on p-GaN for Resonant Cavity Light Emitting Diodes; Mater. Res. Soc. Symp. Proc. Vol. 1288 (2011).

- $\quad$ K. Lee, L. E. Rodak, V. Kumbham, V. Narang, J. S. Dudding, R. Rahimi, S. Kuchibhatla, L. Hornak, D. Korakakis; InGaN MQW LED structures using AlN/GaN DBR and Ag-based pcontact; Mater. Res. Soc. Symp. Proc. Vol. 1288 (2011).

- L. E. Rodak, K. Lee, V. Kumbham, V. Narang, L. A. Hornak, D. Korakakis; Light Emitting Diode Growth on Curved Gallium Nitride Surfaces; Mater. Res. Soc. Symp. Proc. Vol. 1288 (2011). 


\section{BIBLIOGRAPHY}

[1] D. Tromans, "Elastic Anisotropy of HCP Metal Crystals and Polycrystals," International Journal of Research and Reviews in Applied Sciences, vol. 6, p. 462, 2011.

[2] J. Wu, W. Walukiewicz, K. M. Yu, W. Shan, J. W. Ager, E. E. Haller, et al., "Superior radiation resistance of $\operatorname{In}_{1-\mathrm{x}} \mathrm{Ga}_{\mathrm{x}} \mathrm{N}$ alloys: Full-solar-spectrum photovoltaic material system," Journal of Applied Physics, vol. 94, p. 6477, 2003.

[3] M. R. Krames, O. B. Shchekin, R. Mueller-Mach, G. O. Mueller, Z. Ling, G. Harbers, et al., "Status and Future of High-Power Light-Emitting Diodes for Solid-State Lighting," Journal of Display Technology, vol. 3, p. 160, 2007.

[4] O. Ambacher, "Growth and applications of Group III-nitrides," Journal of Physics D: Applied Physics, vol. 31, p. 2653, 1998.

[5] "Department of Energy: Solid State Lighting Research and Development: Multi Year Program Plan," 2014.

[6] Y. Taniyasu, M. Kasu, and T. Makimoto, "An aluminium nitride light-emitting diode with a wavelength of 210 nanometres," Nature, vol. 441, p. 325, 2006.

[7] M. Kneissl, T. Kolbe, C. Chua, V. Kueller, N. Lobo, J. Stellmach, et al., "Advances in group III-nitride-based deep UV light-emitting diode technology," Semiconductor Science and Technology, vol. 26, p. 014036, 2011.

[8] H. Hirayama, "Quaternary InAlGaN-based high-efficiency ultraviolet light-emitting diodes," Journal of Applied Physics, vol. 97, p. 091101, 2005.

[9] M. A. Khan, M. Shatalov, H. P. Maruska, H. M. Wang, and E. Kuokstis, "III-Nitride UV Devices," Japanese Journal of Applied Physics, vol. 44, p. 7191, 2005.

[10] J. Wu, "When group-III nitrides go infrared: New properties and perspectives," Journal of Applied Physics, vol. 106, p. 011101, 2009.

[11] U. K. Mishra, P. Parikh, and W. Yi-Feng, "AlGaN/GaN HEMTs-an overview of device operation and applications," Proceedings of the IEEE, vol. 90, p. 1022, 2002. 
[12] J. R. Srour and J. M. McGarrity, "Radiation effects on microelectronics in space," Proceedings of the IEEE, vol. 76, p. 1443, 1988.

[13] V. Cimalla, J. Pezoldt, and O. Ambacher, "Group III nitride and SiC based MEMS and NEMS: materials properties, technology and applications," Journal of Physics D: Applied Physics, vol. 40, p. 6386, 2007.

[14] M. Benetti, D. Cannata, F. Di Pictrantonio, and E. Verona, "Growth of AlN piezoelectric film on diamond for high-frequency surface acoustic wave devices," Ultrasonics, Ferroelectrics, and Frequency Control, IEEE Transactions on, vol. 52, p. 1806, 2005.

[15] R. C. Turner, P. A. Fuierer, R. E. Newnham, and T. R. Shrout, "Materials for high temperature acoustic and vibration sensors: A review," Applied Acoustics, vol. 41, p. 299 , 1994.

[16] M. A. Dubois and P. Muralt, "Stress and piezoelectric properties of aluminum nitride thin films deposited onto metal electrodes by pulsed direct current reactive sputtering," Journal of Applied Physics, vol. 89, p. 6389, 2001.

[17] A. J. Kenyon, "Recent developments in rare-earth doped materials for optoelectronics," Progress in Quantum Electronics, vol. 26, p. 225, 2002.

[18] P. N. Favennec, H. L'Haridon, M. Salvi, D. Moutonnet, and Y. Le Guillou, "Luminescence of erbium implanted in various semiconductors: IV, III-V and II-VI materials," Electronics Letters, vol. 25, p. 718, 1989.

[19] J. M. Zavada, S. X. Jin, N. Nepal, J. Y. Lin, H. X. Jiang, P. Chow, et al., "Electroluminescent properties of erbium-doped III-N light-emitting diodes," Applied Physics Letters, vol. 84, p. $1061,2004$.

[20] R. G. Wilson, R. N. Schwartz, C. R. Abernathy, S. J. Pearton, N. Newman, M. Rubin, et al., "1.54- $\mu \mathrm{m}$ photoluminescence from Er-implanted GaN and AlN," Applied Physics Letters, vol. 65 , p. 992, 1994.

[21] V. I. Dimitrova, P. G. Van Patten, H. H. Richardson, and M. E. Kordesch, "Visible emission from electroluminescent devices using an amorphous AlN:Er ${ }^{3+}$ thin-film phosphor," Applied Physics Letters, vol. 77, p. 478, 2000. 
[22] S. R. Shannigrahi, F. E. H. Tay, K. Yao, and R. N. P. Choudhary, "Effect of rare earth (La, $\mathrm{Nd}, \mathrm{Sm}, \mathrm{Eu}, \mathrm{Gd}, \mathrm{Dy}, \mathrm{Er}$ and $\mathrm{Yb}$ ) ion substitutions on the microstructural and electrical properties of sol-gel grown PZT ceramics," Journal of the European Ceramic Society, vol. 24, p. 163, 2004.

[23] T. C. Goel, P. K. C. Pillai, H. D. Sharma, A. K. Tripathi, A. Tripathi, C. Pramila, et al., "Effects of rare earth doping on the piezo and pyroelectric properties of lead zirconate titanate (PZT)," in Electrets, 1994. (ISE 8), 8th International Symposium on, 1994, p. 720.

[24] F. Engelmark, G. Fucntes, I. V. Katardjiev, A. Harsta, U. Smith, and S. Berg, "Synthesis of highly oriented piezoelectric AlN films by reactive sputter deposition," Journal of Vacuum Science \& Technology A, vol. 18, p. 1609, 2000.

[25] S. Berg, T. Nyberg, H. O. Blom, and C. Nender, Handbook of Thin Film Process Technology. Bristol, UK: IOP Publishing, 1998.

[26] Y. Zhao, C. Zhu, S. Wang, J. Z. Tian, D. J. Yang, C. K. Chen, et al., "Pulsed photothermal reflectance measurement of the thermal conductivity of sputtered aluminum nitride thin films," Journal of Applied Physics, vol. 96, p. 4563, 2004.

[27] G. A. Slack, L. J. Schowalter, D. Morelli, and J. A. Freitas Jr, "Some effects of oxygen impurities on AlN and GaN," Journal of Crystal Growth, vol. 246, p. 287, 2002.

[28] J. F. Moulder and J. Chastain, Handbook of X-ray Photoelectron Spectroscopy: A Reference Book of Standard Spectra for Identification and Interpretation of XPS Data: Physical Electronics Division, Perkin-Elmer Corporation, 1992.

[29] S. Kohiki, T. Ohmura, and K. Kusao, "Appraisal of a new charge correction method in x-ray photoelectron spectroscopy," Journal of Electron Spectroscopy and Related Phenomena, vol. 31, p. $85,1983$.

[30] L. Rosenberger, R. Baird, E. McCullen, G. Auner, and G. Shreve, "XPS analysis of aluminum nitride films deposited by plasma source molecular beam epitaxy," Surface and Interface Analysis, vol. 40, p. 1254, 2008.

[31] D. A. Shirley, "High-Resolution X-Ray Photoemission Spectrum of the Valence Bands of Gold," Physical Review B, vol. 5, p. 4709, 1972. 
[32] Vibrating Sample Magnetometer (VSM) Option User's Manual 5th ed. San Diego, CA, USA: Quantum Design, 2011.

[33] R. M. d. 1. Rue, R. F. Humphryes, I. M. Mason, and E. A. Ash, "Acoustic-surface-wave amplitude and phase measurements using laser probes," Proceedings of the Institution of Electrical Engineers, vol. 119, p. 117, 1972.

[34] User Manual: Fiber-Optic Vibrometer Sensor Head OFV-551 / -552: Polytec.

[35] J. Harman, A. Kabulski, V. R. Pagán, P. Famouri, K. R. Kasarla, L. E. Rodak, et al., "Effect of contact metals on the piezoelectric properties of aluminum nitride thin films," Journal of Vacuum Science \& Technology B, vol. 26, p. 1417, 2008.

[36] A. Arnau and D. Soares, "Fundamentals of Piezoelectricity," in Piezoelectric Transducers and Applications, ed: Springer, 2008, pp. 1-38.

[37] C. Kittel, Introduction to Solid State Physics: Wiley, 2004.

[38] Y. Sun, T. Nishida, and S. Thompson, "Stress, Strain, Piezoresistivity, and Piezoelectricity," in Strain Effect in Semiconductors, ed: Springer US, 2010, pp. 9-21.

[39] T. Hanada, "Oxide and Nitride Semiconductors: Processing, Properties, and Applications," in Oxide and Nitride Semiconductors, ed: Springer Berlin Heidelberg, 2009, pp. 1-19.

[40] A. Arnau, Y. Jiménez, and T. Sogorb, "Physical Properties of Crystals " in Piezoelectric Transducers and Applications, ed: Springer, 2008, pp. 509-524.

[41] G. Lippmann, " Principe de conservation de l'électricité," Annales de Physique et de Chimie, vol. 5, p. 145,1881 .

[42] H. Jaffe, Piezoelectric Ceramics: Academic Press, 1971.

[43] H. Jaffe and D. A. Berlincourt, "Piezoelectric transducer materials," Proceedings of the IEEE, vol. 53, p. 1372, 1965.

[44] W. J. Meng, "Crystal structure, mechanical properties, thermal properties and refractive index of AlN," in Properties of group III nitrides. vol. 11, J. H. Edgar, Ed., ed: INSPEC, Institution of Electrical Engineers, 1994, pp. 22-29. 
[45] A. Kabulski, V. R. Pagán, and D. Korakakis, "Erbium Alloyed Aluminum Nitride Films for Piezoelectric Applications," MRS Online Proceedings Library, vol. 1129, 2008.

[46] A. H. Morrish, The physical principles of magnetism. New York: IEEE Press, 2001.

[47] V. Narang, D. Korakakis, and M. S. Seehra, "Electronic state of Er in sputtered AlN:Er films determined by magnetic measurements," Journal of Applied Physics, vol. 116, p. 213911, 2014.

[48] M. E. Fisher, "Relation between the specific heat and susceptibility of an antiferromagnet," Philosophical Magazine, vol. 7, p. 1731, 1962.

[49] E. E. Bragg and M. S. Seehra, "Magnetic Susceptibility of $\mathrm{MnF}_{2}$ near $\mathrm{T}_{\mathrm{N}}$ and Fisher's Relation," Physical Review B, vol. 7, p. 4197, 1973.

[50] M. Habenschuss, C. Stassis, S. K. Sinha, H. W. Deckman, and F. H. Spedding, "Neutron diffraction study of the magnetic structure of erbium," Physical Review B, vol. 10, p. 1020, 1974.

[51] R. W. Green, S. Legvold, and F. H. Spedding, "Magnetization and Electrical Resistivity of Erbium Single Crystals," Physical Review, vol. 122, pp. 827-830, 05/01/ 1961.

[52] S. Legvold, "Rare earth metals and alloys," in Handbook of Magnetic Materials: Introduction. vol. 1, E. P. Wohlfarth and K. H. J. Buschow, Eds., ed: North-Holland, 1980, pp. 233-236.

[53] I. S. Jacobs, "Spin-Flopping in $\mathrm{MnF}_{2}$ by High Magnetic Fields," Journal of Applied Physics, vol. 32, p. S61, 1961.

[54] H. Bonrath, K. H. Hellwege, K. Nicolay, and G. Weber, "Antiferromagnetische Umwandlung von $\mathrm{Dy}_{2} \mathrm{O}_{3}, \mathrm{Er}_{2} \mathrm{O}_{3}$ und $\mathrm{Yb}_{2} \mathrm{O}_{3}$ im Temperaturbereich von 1,1 bis $4,2^{\circ} \mathrm{K}, "$ Physik der kondensierten Materie, vol. 4, p. 382, 1966.

[55] R. M. Moon, W. C. Koehler, H. R. Child, and L. J. Raubenheimer, "Magnetic Structures of $\mathrm{Er}_{2} \mathrm{O}_{3}$ and $\mathrm{Yb}_{2} \mathrm{O}_{3}, "$ Physical Review, vol. 176, p. 722, 1968. 
[56] V. Narang, D. Korakakis, and M. S. Seehra, "Nature of magnetism and magnetic-fieldinduced transitions in non-collinear antiferromagnet $\mathrm{Er}_{2} \mathrm{O}_{3}$," Journal of Magnetism and Magnetic Materials, vol. 368, p. 353, 2014.

[57] S. Yang, S. M. Evans, L. E. Halliburton, G. A. Slack, S. B. Schujman, K. E. Morgan, et al., "Electron paramagnetic resonance of $\mathrm{Er}^{3+}$ ions in aluminum nitride," Journal of Applied Physics, vol. 105, p. 023714, 2009.

[58] P. Dutta, A. Manivannan, M. S. Seehra, P. M. Adekkanattu, and J. A. Guin, "Determination of the Electronic State and Concentration of Nickel in NiSAPO Catalysts by Magnetic Measurements," Catalysis Letters, vol. 94, p. 181, 2004.

[59] L. D. Merkle, A. C. Sutorik, T. Sanamyan, L. K. Hussey, G. Gilde, C. Cooper, et al., "Fluorescence of $\mathrm{Er}^{3+}: \mathrm{AlN}$ polycrystalline ceramic," Optical Materials Express, vol. 2, p. 78, 2012.

[60] R. K. Watts and W. C. Holton, "Paramagnetic-Resonance Studies of Rare-Earth Impurities in II-VI Compounds," Physical Review, vol. 173, p. 417, 1968.

[61] E. Bertaut and R. Chevalier, "Magnetic Structure of $\mathrm{Er}_{2} \mathrm{O}_{3}$," Compt. Rend., vol. B 262, p. 1707, 1966.

[62] Y. J. Tang, X. W. Cao, J. C. Ho, and H. C. Ku, "Low-temperature calorimetric study of magnetic ordering in erbium oxide," Physical Review B, vol. 46, p. 1213, 1992.

[63] J. R. Dean and D. Bloor, "Spectroscopy of rare earth oxide systems. II. Spectroscopic properties of erbium oxide (at antiferromagnetic transition)," Journal of Physics C: Solid State Physics, vol. 5, p. 2921, 1972.

[64] H. Choi, S. H. Cho, S. Khan, K.-R. Lee, and S. Kim, "Roles of an oxygen Frenkel pair in the photoluminescence of $\mathrm{Bi}^{3+}$-doped $\mathrm{Y}_{2} \mathrm{O}_{3}$ : computational predictions and experimental verifications," Journal of Materials Chemistry C, vol. 2, p. 6017, 2014.

[65] R. Zhang and R. F. Willis, "Thickness-Dependent Curie Temperatures of Ultrathin Magnetic Films: Effect of the Range of Spin-Spin Interactions," Physical Review Letters, vol. 86, p. $2665,2001$. 
[66] S. Thota, J. H. Shim, and M. S. Seehra, "Size-dependent shifts of the Néel temperature and optical band-gap in $\mathrm{NiO}$ nanoparticles," Journal of Applied Physics, vol. 114, p. 214307, 2013.

[67] P. W. Anderson, "An Approximate Quantum Theory of the Antiferromagnetic Ground State," Physical Review, vol. 86, p. 694, 1952.

[68] K. Ôno, M. Shinohara, A. Ito, N. Sakai, and M. Suenaga, "Observation of Zero-Point Spin Deviation in Antiferromagnetic $\mathrm{K}_{3} \mathrm{Fe}(\mathrm{CN})_{6}$," Physical Review Letters, vol. 24, p. 770, 1970.

[69] Y. Shapira and S. Foner, "Magnetic Phase Diagram of $\mathrm{MnF}_{2}$ from Ultrasonic and Differential Magnetization Measurements," Physical Review B, vol. 1, pp. 3083-3096, 1970.

[70] D. J. Simkin, "Mössbauer Study of Metamagnetic Transition in $\mathrm{FeCl}_{2}$ and $\mathrm{FeBr}_{2}$," Physical Review, vol. 177, p. 1008, 1969.

[71] B. R. Heap, "Application of the Bethe-Peierls Method to a Metamagnetic, $\mathrm{FeCl}_{2}$," Proceedings of the Physical Society, vol. 80, p. 248, 1962.

[72] J. D. Rall, M. S. Seehra, and E. S. Choi, "Metamagnetism and nanosize effects in the magnetic properties of the quasi-two-dimensional system $\beta-\mathrm{Ni}(\mathrm{OH})_{2}, "$ Physical Review B, vol. 82, p. 184403, 2010.

[73] S. G. E. te Velthuis, J. S. Jiang, S. D. Bader, and G. P. Felcher, "Spin Flop Transition in a Finite Antiferromagnetic Superlattice: Evolution of the Magnetic Structure," Physical Review Letters, vol. 89, p. 127203, 2002.

[74] G. Chaddha and M. S. Seehra, "Magnetization process and principal magnetic susceptibilities in $\mathrm{RbMnF}_{3}$," Solid State Communications, vol. 44, p. 1097, 1982.

[75] W. J. Ince, "Coupled Antiferromagnetic-Nuclear-Magnetic Resonance in $\mathrm{RbMnF}_{3}$," Physical Review, vol. 184, p. 574, 1969.

[76] Y. Shapira, "Tetracriticality of $\mathrm{RbMnF}_{3}$," Journal of Applied Physics, vol. 52, p. 1926, 1981.

[77] M. S. Seehra and T. G. Castner, "Study of the Ordered Magnetic State of Copper Formate Tetrahydrate by Antiferromagnetic Resonance," Physical Review B, vol. 1, p. 2289, 1970. 
[78] G. Schäfer, "Paramagnetische Resonanz von $\mathrm{Er}^{3+}$ und $\mathrm{Ce}^{3+}$ in $\mathrm{Y}_{2} \mathrm{O}_{3}$, " Physik der kondensierten Materie, vol. 9, p. 359, 1969. 